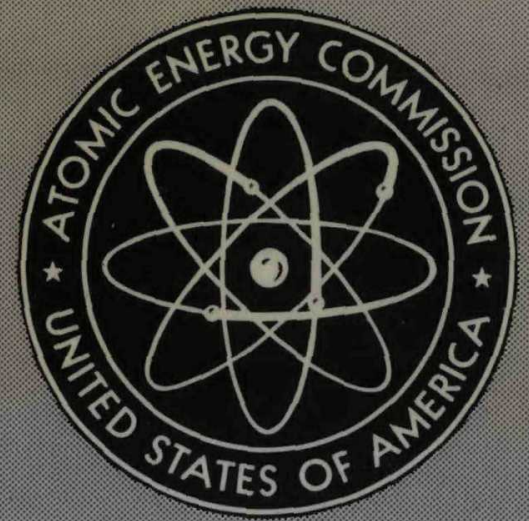

WCAP - 2347

SODIUM PUMP DEVELOPMENT AND PUMP TEST FACILITY DESIGN

August 1963

Atomic Power Division

Westinghouse Electric Corporation

Pittsburgh, Pennsylvania 


\section{DISCLAIMER}

This report was prepared as an account of work sponsored by an agency of the United States Government. Neither the United States Government nor any agency Thereof, nor any of their employees, makes any warranty, express or implied, or assumes any legal liability or responsibility for the accuracy, completeness, or usefulness of any information, apparatus, product, or process disclosed, or represents that its use would not infringe privately owned rights. Reference herein to any specific commercial product, process, or service by trade name, trademark, manufacturer, or otherwise does not necessarily constitute or imply its endorsement, recommendation, or favoring by the United States Government or any agency thereof. The views and opinions of authors expressed herein do not necessarily state or reflect those of the United States Government or any agency thereof. 


\section{DISCLAIMER}

Portions of this document may be illegible in electronic image products. Images are produced from the best available original document. 


\section{LEGAL NOTICE}

This report was prepared as an account of Government sponsored work. Neither the United States, nor the Commission, nor any person acting on behalf of the Commission:

A. Makes any warranty or representation, expressed or implied, with respect to the accuracy, completeness, or usefulness of the information contained in this report, or that the use of any information, apparatus, method, or process disclosed in this report may not infringe privately owned rights; or

B. Assumes any liabilities with respect to the use of, or for damages resulting from the use of any information, apparatus, method, or process disclosed in this report.

As used in the above, "person acting on behalf of the Commission" includes any employee or contractor of the Commission, or employee of such contractor, to the extent that such employee or contractor of the Commission, or employee of such contractor prepares, disseminates, or provides access to, any information pursuant to his employment or contract with the Commission, or his employment with such contractor.

This report has been reproduced directly from the best available copy.

Printed in USA. Price $\$ 4.00$. Available from the Clearinghouse for Federal Scientific and Technical Information, National Bureau of Standards, U. S. Department of Commerce, Springfield, Va. 
SODIUM PUMP DEVELOPMENT

AND

PUMP TEST FACILITY DESIGN

Prepared for the New York Operations Office

U. S. Atomic Energy Commission

Under AEC Contract AT(30-1)-3123

August, 1963

Contributors

H. G. Allen, WAED

J. Boyd, W Research Laboratories

B. Cametti, WAED

D. A. Maniero

D. R. Nixon, WAED

A. A. Raimondi, W Research Laboratories

C. C. Randall, Project Engineer

J. G. Wilhite, WAED

APPROVED:

J F. Wright, Ganager

Advanced Reactor Systems
APPROVED:

B. Camett1

Engineering Consultant

WAED

WESTINGHOUSE ELECTRIC CORPORATION

Atomic Power Division

P. O. Box 355

Pittsburgh, Pa. 15230 


\begin{abstract}
The study defines a program for the development of large sodium pumps for use with sodium cooled reactor systems of 1000-1500 MWe capability, and the functional requirements of a related sodium pump test facility for testing large pumps. The future pump requirements of large power systems have been estimated, a type of sodium pump recommended for further development, the development problems identified and a program research and development prepared to resolve these problems. The functional requirements of a sodium pump test facility for testing pumps for large reactor use have been established.
\end{abstract}


TABLE OF CONTENTS

Page Number

ABSTRACT $-\cdots \cdots-\ldots-\ldots-\ldots-\ldots$ iv

TABLE OF CONTENTS

v

LIST OF FIGURES - - - - - - - - - - - - -

$\mathbf{x i}$

LIST OF TABLES - - $-\ldots+\ldots$

xii

1.0 SUMMARY $-\ldots+\ldots-\ldots$

1

2.0 INTRODUCTION $-\ldots-\ldots-\ldots-\ldots-\ldots-\ldots-\ldots$

2.1 BACKGROUND - $\ldots+\ldots$

2.2 OBJECTIVES - $-\ldots-\ldots-\ldots$

2.3 ORGANIZATION OF REPORT - - - - - - - - - 6

3. 0 BACKGROUND OF SODIUM PUMP TECHNOLOGY - - - - - -

3.1 SODIUM PUMP DEVELOPMENTS - - - - - - - - 6

3.2 SODIUM PUMP TYPES $-\ldots-\cdots$

3.2.1 Mechanical Pump with Shaft Seals - - - - - 9

3.2.2 Mechanical Pump without Shaft Seals - - - - 9

3.2.3 Electromagnetic Pumps - - - - - - - 10

3.2.4 Mechanical Electromagnetic Pumps - - . - - - 10

3. 3 SUMMARY OF CURRENT SODIUM PUMP TECHNOLOGY - - - 10

4. 0 FUTURE SODIUM PUMP REQUIREMENTS - - - - - - - - 12

4. 1 SYSTEMS DEPENDENCY - - - - - - - - - 12 
TABLE OF CONTENTS (CONT )

Page Number

4.1.1 Flow $\ldots \ldots \ldots$

4.1.2 Pump Head $\ldots \ldots$

4.1.3 Pumping Fluid Temperature $\ldots \ldots$

4.2 PRIOR DESIGN CRITERIA $-\cdots \cdots$

4.3 ESTIMATE OF FUTURE PUMP HYDRAULIC REQUIREMENTS -

4.4 FUTURE PUMP FUNCTIONAL REQUIREMENTS - - - -

Do

4.4.1 General $\ldots \ldots \ldots$

\section{0}

4.4.2 Containment and Isolation of Sodium - - - - -

4.4.3 Maintenance - - - . - . - . - -

4.4.4 Flow Control - - - - - - - - - -

4.4.5 Layout Compatibility $-\cdots-\ldots$

5.0 SELECTION OF PUMP FOR FURTHER DEVELOPMENT - - - -

5.1 CRITERIA $\ldots \ldots \ldots$

5.2 RELIABILITY CONSIDERATIONS - - - - - - - -

\section{2}

5.2.1 Pump End … . . . . . . . -

5.2.2 Drive $\ldots \ldots \ldots \ldots$ 25

5.2.3 Bearings $\ldots \ldots \ldots$

\section{6}

5.2 .4 Seals $\ldots \ldots \ldots$

5.2.5 Auxiliaries $\ldots \ldots \ldots$

$5.3{ }^{\circ} \cos T \ldots \ldots \ldots \ldots \ldots \ldots$

5.3.1 Operating Costs $\ldots \ldots \ldots \ldots$

5.3.2 Initial Costs $\ldots \ldots \ldots \ldots$ 
TABLE OF CONTENTS (CONT.)

Page Number

5.4 DESIGN PREDICTABILITY - - - - - - - - - - 34

5.5 SYSTEMS CONSIDERATIONS - - - - - - - - 35

5.5 .1 Layout - - - - - - - - - - - 35

5.5.2 Flow Control - - - - - - - - 37

5.5.3 Shielding - - $\ldots \ldots$ - 39

5.5.4 Safety - - - - - - - - - 39

5.6 SELECTION OF TYPE FOR FUTURE DEVELOPMENT - - - 41

6. 0 PUMP DESIGN RECOMMENDED FOR DEVELOPMENT - - - - 44

7.0 OVERALL DEVELOPMENT PROGRAM - - - - - - - 45

7.1 SCOPE $--\ldots-\ldots-\ldots-\ldots 45$

7. 2 DEVELOPMENT PROGRAM AND SCHEDULE - - - - - 45

7.2.1 Plant Construction Schedule - - - - - - - 47

7.2.2 Development Program Schedule - - - - - 47

7.3 SUPPORTING ACTION $-\ldots-\ldots-\ldots 3$

7.4 SODIUM PUMP DEVELOPMENT COSTS - - - - - - - 54

8.0 DEVELOPMENT PROGRAM FOR MAJOR PROBLEM AREAS - - 57

8.1 SCOPE $\ldots \ldots-\ldots . \ldots 7$

8.2 SHAFT SEAL DEVELOPMENT $\ldots \ldots-\ldots 7$

8.2.1 Discussion of Problems $-\ldots-\ldots 7$

8.2.2 Experimental Program - - - - - - - 63

8.2.3 Schedule and Cost $-\ldots-\ldots 4$ 


\section{TABLE OF CONTENTS (CONT.)}

Page Number

8.3 BEARINGS DEVELOPMENT - - - - - - - - - - 64

8.3.1 Discussion of Problems - - - - - - - 64

8.3.2 Bearing Development Program - - - - - - 69

8.3.3 Schedule and Cost $-\ldots-\ldots 2$

8.4 PUMP HYDRAULIC DEVELOPMENT - - - - - - - - 72

8.4.1 Discussion of Problems $-\ldots-\ldots 2$

8.4.2 Pump Development Program - - - - - - - 74

8.4.3 Schedule and Cost - - $-\ldots-\ldots 74$

9.0 ENGINEERING ANALYSIS OF ANCILLARY PROBLEMS - - - - 76

9.1 GENERAL $-\ldots-\ldots-\ldots-\ldots$

9.2 THERMAL STUDIES $-\ldots-\cdots-\cdots$

9.2.1 Problems $-\ldots-\ldots-\ldots$

$9.2,2$ Required Analysis and Testing - - - - - - 76

9.3 FLUID STABILITY STUDIES - - - - - - - - - - 77

9.3.1 Problems $--\ldots-\ldots-\ldots 7$

9.3.2 Required Analysis and Testing - $-\ldots-\ldots$

9.4 DYNAMICS AND VIBRATION STUDIES- - - - - - - - - 77

9.4.1 Problems - - - - - - - - - -47

9.4.2 Required Analysis and Testing - - - - - - 78

9.5 MECHANICAL STUDIES $-\ldots-\ldots$

9.5.1 Problems $-\ldots-\ldots 78$

9.5.2 Static Seal Development and Testing - - - - - 78 
9.6 MATERIALS SELECTION $-\cdots-\cdots$ 9.6.1 Problems $\ldots \ldots \ldots$

9.6.2 Required Analysis and Testing - . - . - - 80

9.7 ELECTRICAL DESIGN STUDIES $\ldots \ldots$

9.7.1 Problems $\ldots \ldots-\ldots$

9.7.2 Required Analysis and Testing - - - - - 81

9.8 INSTRUMENTA TION AND CONTROL - - - - - - - 81

9.8.1 Problems $\ldots \ldots-\ldots-\ldots$

9.8.2 Required Analysis and Testing _. - . - . 81

9.9 SCHEDULE AND COST $-\ldots-\ldots, \ldots$

10.0 SODIUM PUMP TEST FACILITY REQUIREMENTS - - - - 84

10.1 GENERAL $\ldots \ldots-\ldots 8$

10.2 FUNCTION $\ldots \ldots \ldots 84$

10.3 TEST CAPABILITIES $-\ldots-\ldots, \ldots-\ldots$

10.3.1 General Considerations - - . - . - 84

10.3.2 Steady State Hydraulic Test $\ldots \ldots-\ldots$

10.3.3 Hydraulic Transients $\ldots \ldots-\ldots$

10.3.4 Thermal Transients $\ldots \ldots \ldots-\ldots$

10.3.5 Flow Control with Pumps - - . - . - 86

10.3.6 Component Evaluation - . - . . . 86

10.3.7 Life Tests and Accelerated Wear Tests - - - 87 
TABLE OF CONTENTS (CONT.)

Page Number

10.4 OPERATING RANGE FOR SODIUM PUMP TEST

FACILITY - - - - - - - - - - - - 87

10.5 GENERAL CONSIDERATIONS - $\ldots \ldots 8$

10.5.1 Flow Loops - - - - - - - - - $-{ }_{-} 88$

10.5.2 Temperature Adjustment - . - . - . - 88

10.5.3 Transient Simulation - - - - - - - - 88

10.5.4 Flow Measurement - - - - - - - - - 88

10.5.5 Flow Resistance - - - - - - - - - 88

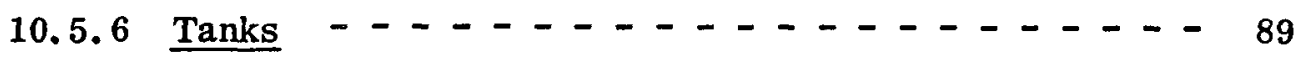

10.5.7 Pump Test Bed $-\cdots-\ldots-\cdots$

10.5.8 Supporting Systems and Services - - - - - 89

11.0 PRELIMINARY CONCEPT OF SODIUM TEST FACILITY - - - - - 91

APPENDIX 1, WAED EM 3131, LARGE LIQUID METAL PUMP STUDY HYDRAULICS DESIGN \& DEVELOPMENT

APPENDIX 2, WAED EM 3123, SODIUM PUMP DESIGN STUDY DRIVE MOTOR STUDY

APPENDIX 3

RECENT OPERATING DIFFICULTIES OF PRIMARY SODIUM PUMPS IN EBR II \& HNPF 


\section{LIST OF FIGURES}

Figure Number

1

2

3

4

5

6

7

8

9

10

$10 \mathrm{a}$

$10 \mathrm{~b}$

11

12

13

14

15

16

17

18

19

20 $\underline{\text { Title }}$

Pump Types

Sodium Pump Parameters

Features of Mechanical, Electromagnetic and

Mechanical Electromagnetic Pumps

Overall Pump Efficiency vs 20 Year Power Cost

Basic Primary Loop Arrangements

Drive Efficiencies

Conceptual Drawing of a 6000 HP Free Surface

Shaft Sealed Sodium Pump

Overall Sodium Pump Development Program

Sodium Pump Development Program Pert Network

Overall

Phase I Sodium Pump Development Program Pert Network - Seal \& Bearing Development

Phase I Sodium Pump Development Program Pert Network - Development Pump DS-1

Phase I Sodium Pump Development Program Pert Network - Development Pump DS-2

Seal Types

Seal Experimental Program Schedule and Cost

Comparison of Hydrostatic \& Pivoted-pad

Journal Bearings

Bearing Development Program Schedule and Cost

Pump Hydraulic Development Program Schedule and Cost

Lower End of Pump and Tank Showing Static Seal Area

Engineering Analysis of Ancilliary Problems

82

Preliminary SPTF Flow Diagram

92

Artists Concept of SPTF Two Pump Arrangement

93

Artists Concept of SPTF Large Single Pump

94

19

23

32

36

40

43

46

49

50

$50 \mathrm{a}$

$50 \mathrm{~b}$

59

65

67

73

75

79

Arrangement 


\section{LIST OF TABLES}

Table Numbers

1

2

3

4

5

6

7

8

9
Title

Sodium Cooled Reactors

Current Designs

Survey of Future Liquid Metal (Na or NaK)

Pump Requirements

Pump Requirements

20 Year Energy Costs

Pump - Reactor Arrangement Summary

Comparative Pump Ratings

Cost Estimate

Materials and Methods of Application
Page Number

7

15

16

17

31

38

42

48

70 


\subsection{SUMMARY}

While many nuclear reactor systems have been shown to be technically capable of producing power, long range forecasts for the 1970's emphasize the economic importance of large $(1000-1500 \mathrm{MWe})$ plants. The successful utilization of sodium cooled reactors for this service depends upon the development and testing of large plant components including pumps. This report investigates the requirements of pumps for this service and the related development program and test facility necessary to their development.

Sodium pumps may be classified into three general types: mechanical, electromagnetic and a combination of the two, mechanical-electromagnetic. Mechanical liquid sodium pumps have been constructed with fluid horsepower ratings from 2-1000 HP with capacities to $13,000 \mathrm{GPM}$ with efficiencies to $77 \%$. A majority of these pumps use oil lubricated mechanical face type gas seals shaft seals. Almost all the primary and secondary pumps in reactor service today are of this type. A large number of electromagnetic pumps have been constructed predominantly in small sizes, although capacities to 10,000 GPM with fluid HP ratings to $440 \mathrm{HP}$ have been built and tested. Efficiencies as high as $43 \%$ have been obtained on large AC linear induction and DC conduction type pumps. Promising development work is currently underway on mechanical-electromagnetic pumps of the helical rotor type and pumps of this type rated at 1000 fluid horsepower and capacities to 3000 GPM have been tested with efficiencies to $28 \%$.

Contrary to experience with other types of sodium components, pump performance to date has been essentially up to expectations and malfunctions have been comparatively few. Difficulties have been reported primarily in the area of seal and bearing design, which seem to be amenable to correction with further development. The present status of pump technology appear to offer a sound base from which to proceed to the development of larger pumps for future requirements. The current status of sodium pump technology has previously been reported in the topical report WCAP-2255, Survey of Sodium Pump Technology, and is summarized in the body of this report.

Because the pumps are but one component of a complex interrelated system, the functional requirements are dictated by many factors, among which are reactor core design and overall economic consideration. Experience with other reactor systems has indicated that improvement in the economics may be expected by increasing heat extraction per unit of coolant flow and by minimizing the number of coolant flow loops. Opposing this trend are safety limitations imposed by the sensitivity of reactor core design to increased heat removal per unit of coolant and requirement of adequate cooling upon failure of a single heat transfer loop. From a review of current designs and future trends in conjuction with the magnitude of the various functional parameters, it is concluded that it may be reasonably expected that future large reactor plants will require pumps with a capacity of 50,000 to 100,000 GPM developing heads of 200 to 350 feet of fluid. 
An evaluation of the relative reliability, design predictability, cost, both initial and operating, and consideration of reactor systems requirements led to the selection of the mechanical shaft sealed pump as the most desirable type of pump for development for future reactor use. The preferred design selected is based on the "free surface" concept in which a interface is maintained in the pump casing between the liquid sodium and an inert cover gas. A shaft seal is provided to maintain the inert gas atmoshpere within the pump tank. The concept utilizes a sodium lubricated radial shaft bearing. A promising alternate is a totally enclosed motor pump in which the shaft seal is not required and in which the sodium is isolated from the motor cavity by inert gas pressure in the enclosed motor casing.

The successful operation of mechanical "free surface" shaft sealed pumps rated at 1000 fluid HP indicates the general feasibility of the concept. However, further development is indicated to be necessary in the key areas of seal design, bearing design and improved pump hydraulics before the concept can be reliably scaled up to future requirements.

An overall pump development program totaling approximately $\$ 22,000,000$ to be carried out over a period of 12 years is defined. The program includes development of the fundamental design characteristics of critical pump components, the design and fabrication of development pumps of various sizes and types for testing and the cost of testing including the construction and operation of a Sodium Pump Test Facility.

In the area of sodium lubricated bearings the program provides for the determination of basic performance characteristics of both hydrostatic bearings and pivoted pad journal bearings; the selection, application, and fabricability of bearing materials and the accelerated wear testing of materials under stopstart and thermal shock conditions; and the measurement of radial bearing loads model testing of proposed pump designs.

The program requires the construction and model testing of both single and double suction pumps in order to optimize hydraulics and minimize both radial bearings loads and required net positive suction heads.

The program also provides for analysis of ancilliary design problems related to flow control, fluid stability studies, thermal stress and expansion problems, dynamics and vibration analysis and instrumentation and control requirements.

Although the development program provides for testing and development of individual pump components, prudence dictates that full scale demonstration and proof testing of a prototype pump is necessary and desireable prior to installation in a major power reactor. The synergistic effects of individually tested model components on overall pump performance are traditionally unpredictable. Even 


\subsection{SUMMARY (CONT.)}

though firm price and performance guarantees might be obtained from suppliers, consequential damages resulting from pump failure are not recoverable. A full scale flexible sodium pump test facility to provide for full scale testing of development pumps and the demonstration and proof testing of reactor pumps is indicated.

A flexible sodium pump test facility must provide for testing: (1) pump characteristics, (2) speed/flow control response, (3) "thermal shock resistance", (4) performance under extended operational tests both in steady state and cyclic flow conditions.

To provide for the necessary testing of pumps required for future large reactor use such a facility must provide flow capability to 120,000 GPM, operating sodium temperature over the range of $400^{\circ} \mathrm{F}$ to $1200^{\circ} \mathrm{F}$, provision for induced thermal transients of $10^{\circ} \mathrm{F} /$ second, variable net positive suction head up to 50 psig and variable discharge heads of up to 350 feet of sodium.

An initial concept of a sodium pump test facility to meet the genefal requirements of the program provides for two independent parallel 60,000 GPM test loops. Each test loop incorporates a pump test stand, a 5 MWe pump power supply, a sodium flow measurement device, a flow control valve, a sodium to air heat sink, and a sodium head tank. A drain tank common to both loops is provided. Thermal transients will be induced in one loop at a time by a feed and bleed system from the alternate loop operated at a different temperature. The concept includes a third pump test stand that will permit installation of a 120,000 GPM pump which will utilize both loops in parallel to provide the necessary flow capacity.

The overall test facility will include necessary auxiliary systems and equipment to provide for heating, inert gas pressurization, sodium purification, and pump handling and inspection.

The preliminary design and cost estimate of a sodium pump test facility will be investigated in Phase II (See Page 5) of the contract and reported separately. 


\section{$2.0 \quad$ INTRODUCTION}

\subsection{BACKGROUND}

While many nuclear reactor systems have been shown to be technically capable of producing power, long range forecasts of power demands for the 1970's and nuclear system cost trends emphasize strongly the economic importance of larger (1000-1500 MWe) plants for future utility application. The successful utilization of sodium cooled reactors for this service depends to an important degree upon the development and testing of larger and more economic system components. Key components include sodium-sodium heat exchangers, sodium steam generators, sodium circulating pumps and related flow measurement and control devices. An extensive program for the design and development of sodium heat exchangers, and for the construction and operation of a facility in which to test them is being carried out by the AEC as an important step toward the development of large sodium components. This study investigates the requirements and scope of a similar type and complementary program for the development and testing of large sodium pumps and related flow components.

\subsection{OBJECTIVES}

This study was undertaken for the purpose of surveying and presenting the fundamental technical and economic information that is essential for the planning of a long range two phases; the first phase was concerned primarily with the establishment of program objectives and requirements, and the second phase was concerned with a conceptual preliminary design and test program (with costs thereof) for a test facility for large sodium pumps. The specific objectives of the study, by phases, are as follows:

Phase I ---- Sodium Pump Development Program

1. Establishment of tentative engineering requirements for sodium pumps compatible with large plant objectives.

2. Identification of specific pump development problems that must be solved.

3. Specification of a pump development program to implement the solution of identified problems.

4. Establishment of functional requirements of an experimental test facility for testing and demonstration of large sodium pumps. 


\subsection{OBJECTIVES (CONT.)}

Phase II ---- Pump Test Facility Design and Cost

1. Preparation of a conceptual preliminary design of an experimental sodium pump test facility.

2. Preparation of a cost estimate and design schedule for design and construction of the facility.

3. Preparation of a preliminary test program and estimate of facility operating cost.

\subsection{ORGANIZATION OF REPORT}

This report encompasses only the work of Phase I of Contract AT(30-1)-3123 with the USAEC New York Operations Office. The work to be performed under Phase II will be separately reported at the completion of the contract.

This report includes, as Section 3, a summary of the current status of sodium pump technology which was abstracted from a topical report* prepared earlier in the study and issued separately.

\footnotetext{
*WCAP-2255 Survey of Sodium Pump Technology
} 


\subsection{BACKGROUND OF SODIUM PUMP TECHNOLOGY}

\subsection{SODIUM PUMP DEVELOPMENTS}

Over the past 15 years, the pumps for circulating liquid metals for reactor cooling have been designed and built as the needs arose. For early experimental reactor and heat transfer work, pumps of various designs were provided, but only a minimum of emphasis was placed on pump design. As the reactor technology progressed, so did the need for large and more reliable liquid metal pumps. Both mechanical and electromagnetic pumps with capacities up to 10,000 GPM have already been built for the reactors listed in Table 1. The general requirements which have affected the design of sodium pumps for these reactor plants are:

1. High temperature

2. Leakless or near leakless operation

3. Low maintenance

4. Good efficiency

5. Adequate NPSH

6. Size, weight and pump location

7. Reliability

\subsection{SODIUM PUMP TYPES}

From a purely hydraulic standpoint, the mechanics of pumping sodium is similar to pumping water. In addition, the good electrical conductivity and permeability of liquid metals makes it possible to pump sodium by electromagnetic means.

Sodium pumps may be classified by types as shown in Figure 1. The subsequent parts of this section will deal with various types in accordance with the classification.

The mechanical pumps are usually centrifugal units with a suitable rotating drive equipped with either a shaft seal or an enclosed motor to prevent sodium or inert cover gas leakage.

Electromagnetic pumps are units which produce a pumping force by the interaction of a current passed through the liquid metal and a magnetic field. They have no moving parts other than the flowing liquid and are completely hermetic. 
SODIUM COOLED REACTORS

\begin{tabular}{|c|c|c|c|c|c|c|c|c|}
\hline \multirow[b]{2}{*}{ DATE } & \multirow[b]{2}{*}{ REACTOR } & \multirow[b]{2}{*}{ LOCATION } & \multirow[b]{2}{*}{ OPERATOR } & \multirow[b]{2}{*}{ TYPE } & \multirow{2}{*}{ POWER (MW) } & \multirow{2}{*}{$\begin{array}{c}\text { COOLANT } \\
\text { TEMP. } \\
\text { MAX. }\end{array}$} & \multicolumn{2}{|l|}{ PUMPS } \\
\hline & & & & & & & PRIMARY & SECONDARY \\
\hline 1951 & $E B R-1$ & ANL & AEC & TEST & $1.5 /-$ & $\begin{array}{l}\operatorname{Mak} \\
662{ }^{\circ} \mathrm{F}\end{array}$ & $I D C E M$ & -- \\
\hline 1955 & $\$ 1 G$ & MEST MILTOM & KAPL & SUB PROTO & & $\mathrm{Ma}$ & 1 & 1 \\
\hline 1956 & $\$ 2 G$ & SEAWOLF & USN & SUB PROP. & & $\mathrm{Ma}$ & $1 \quad 1$ & $(1)$ \\
\hline 1957 & SRE & $\begin{array}{l}\text { SAMTA SUSAMA, } \\
\text { CALIF. }\end{array}$ & $\triangle E C / A 1$ & POWER & $20 / 62$ & ${ }_{9600 \mathrm{~F}}^{M a}$ & $\begin{array}{l}\text { 2-MECH FREEZE } \\
\text { SEAL }\end{array}$ & $\begin{array}{l}\text { 2-MECH. FREEZE } \\
\text { SEAL }\end{array}$ \\
\hline 1957 & BR -5 & OBNINSK & (RUSSIA) & TEST & $5 /-$ & $\mathrm{Ma}$ & -- & -- \\
\hline 1959 & SNAP 2 & & $A 1$ & & -- & Ma & $\begin{array}{l}\text { PERM. MAGKET } \\
\text { EM-PUMPS }\end{array}$ & \\
\hline 1959 & ORF & $\begin{array}{l}\text { DOUNREAY, } \\
\text { ENGLAND }\end{array}$ & AEA & POWER & $60 / 15$ & $\begin{array}{l}\operatorname{MaK} \\
662^{\circ} \mathrm{F}\end{array}$ & $24-A C$ EM & $24-A C$ EM \\
\hline 1959 & $B N-50$ & ULYANOYSKI & (RUSSIR) & POWER & -150 & Ma & & \\
\hline 1961 & KFAPP & DETROIT & PRDC & POWER & $300 / 100$ & ${ }_{8000}^{\mathrm{Ma}}$ & $\begin{array}{l}\text { 3-MECH FREE SURFACE } \\
\text { GAS SEAL }\end{array}$ & 3-MECH - GAS SEAL \\
\hline 1961 & $E B R-11$ & MRTS & AEC & POWER & $62 / 20$ & $\stackrel{\mathrm{Ma}}{900^{\circ} \mathrm{F}}$ & $\begin{array}{l}\text { 2-CANMED MOTOR. } \\
\text { TOTALLY ENCLOSED }\end{array}$ & $1-1 C E M$ \\
\hline 1961 & LAMPRE I & LASL & AEC & TEST & $1 /-$ & $\begin{array}{l}\mathrm{Ma} \\
9000^{\circ} \mathrm{F}\end{array}$ & $2-E M$ & \\
\hline 1961 & HNPF & HALLAM, MEBR. & CPPD & POWER & $240 / 76$ & $\begin{array}{l}\mathrm{Ma} \\
945^{\circ} \mathrm{F}\end{array}$ & 3-MECH GAS SEAL & 3-MECH GAS SEAL \\
\hline 1953 & RAPSODIE & $\begin{array}{l}\text { CADARACHE, } \\
\text { FRAMCEE }\end{array}$ & CEA & TEST & $10 /-$ & $\begin{array}{l}M a \\
645 \circ\end{array}$ & -- & --- \\
\hline
\end{tabular}

TABLE 1 


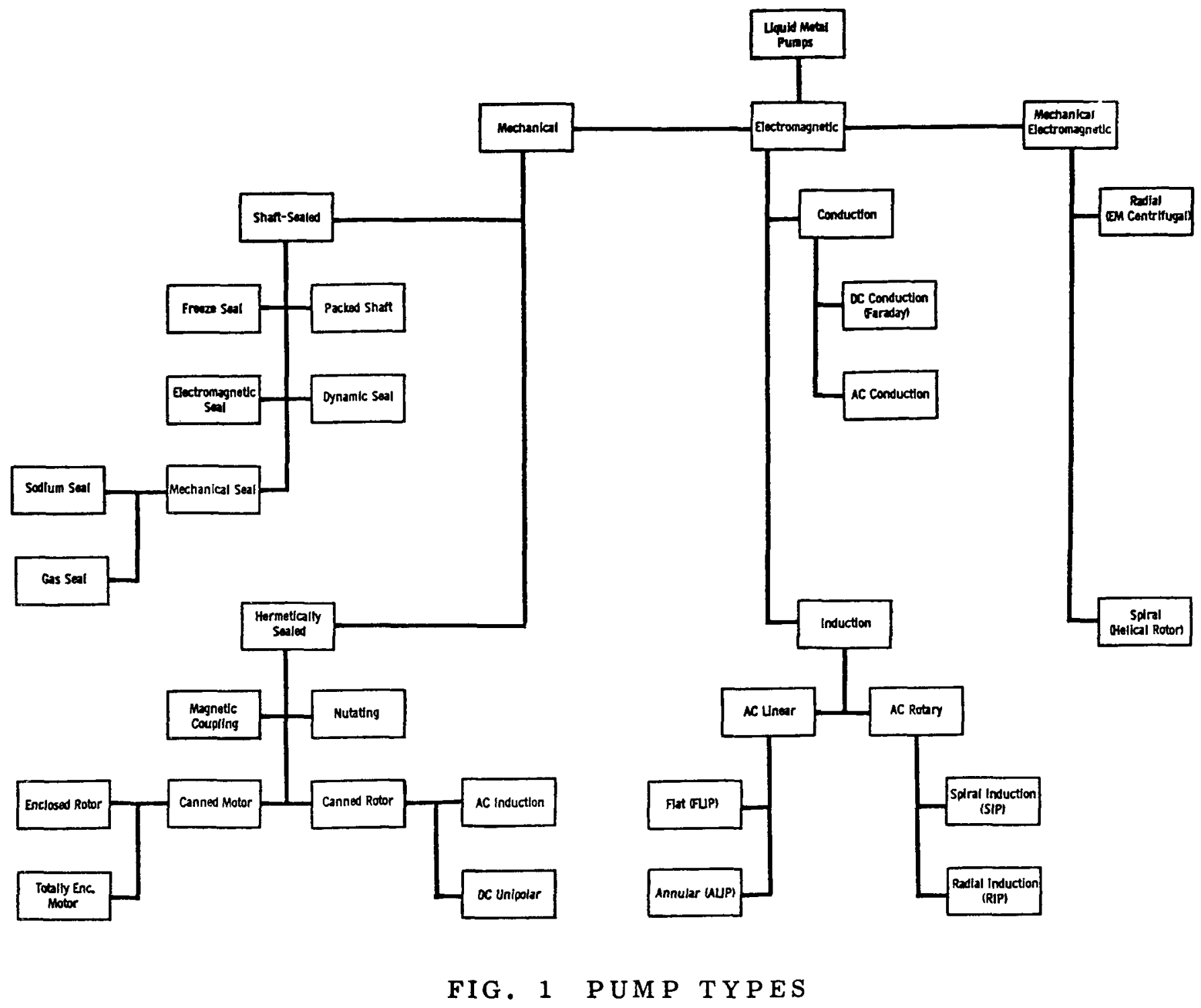




\section{2 SODIUM PUMP TYPES (CONT)}

Mechanical electromagnetic pumps combine both mechanical and electromagnetic principles by mechanically moving a magnetic field in the proximity of a conductive liquid. The field travels through the liquid and induces eddy currents within the liquid which interact with the magnetic field to produce a force on the liquid.

\subsubsection{Mechanical Pumps With Shaft Seals}

Mechanical pumps with some form of shaft seal are a widely used and efficient means of pumping liquid sodium. The most commonly used design consists of a vertical centrifugal pump operating with a "free surface" of sodium in the pump casing. A mechanical shaft seal separates a low pressure inert gas covering the surface of the sodium from the normal atmosphere.

Pumps of this design are characterized by conventional centrifugal pump hydraulic performance and efficiency and utilize conventional drive mechanisms. The disadvantages associated with this type of pump for reactor service are primarily the restriction on location and attitude required to maintain a free surface in the casing and the inherent limitations of flow control by conventional drive systems.

Maintenance requirements are primarily those associated with the shaft seals. For oil lubricated face type seals, six months to a year of service life without maintenance is typical.

\subsubsection{Mechanical Pumps Without Shaft Seals}

Mechanical pumps without shaft seals have been built for liquid metal service in a variety of configurations. In general the pumped fluid and pump rotor is. separated from the driving mechanisms by a membrane or "can". The drive is usually effected by electromagnetic coupling.

The advantages of this class of pumps are the complete absence of moving seals, relative compactness of design, and independence of the attitude limitations of "free surface" designs.

This class of pumps has the disadvantage in general of achieving somewhat lower efficiencies than shaft sealed pumps and requires special motor designs with inherently difficult speed control characteristics ind higher costs.

The lack of moving seals indicate a potentially favorably maintenance condition for this type of pump, providing adequate bearing life is achieved. 


\subsubsection{Electromagnetic Pumps}

The good electrical conductivity and permeability of liquid metals makes it possible to pump these materials by electromagnetic means. A large number of types and configurations of electromagnetic pumps have been designed and constructed for sodium service, including both induction and conduction types utilizing both $\mathrm{AC}$ and DC.

In general, the electromagnetic pumps provide a means of achieving direct flow control, hermetically sealed flow systems, freedom from attitude limitations, and systems without moving parts.

In practice, these advantages have been attained at the expense of relatively low efficiency, heavy bulky units, and frequency very special power supply systems.

Maintenance of electromagnetic systems is quite low but thermal and radiation damage to electrical insulation are significant problems.

\subsubsection{Mechanical Electromagnetic Pumps}

A number of novel designs have been investigated that utilize some aspects of both the mechanical type pumps and the electromagnetic pumping principle. These designs have been attempts to incorporate the better features of each type of pump.

Generally these types incorporate the closed sodium liquid system and an electromagnetic pumping with the use of a mechanically progressed magnetic field driven by a conventional motor drive.

Results to date indicate that moderate efficiencies can be attained in pumps that have no moving parts in contact with sodium and which do not require unusual power supplier.

Disadvantages include the requirement of a thin membrane for containment of the pump sodium and problems of thermal protection of electrical systems.

\subsection{SUMMARY OF CURRENT SODIUM PUMP TECHNOLOGY}

The current status of sodium pump technology as related to use in the nuclear power industry may be summarized as follows:

1. Over 75 mechanical liquid sodium pumps have been constructed as of March, 1963. The fluid horsepower ratings range from 2-1000 HP with capacities up to 13,000 GPM. Over half of these units employ oil lubricated mechanical face type gas shaft seals. Maximum efficiency obtained is $77 \%$. 


\subsection{SUMMARY OF CURRENT SODIUM PUMP TECHNOLOGY (CONT.)}

2. Over 400 electromagnetic sodium pumps have been constructed as of March, 1963. The fluid horsepower ratings range from less than 1-440 HP with capacities up to 10,000 GPM. More than half of these units are small (less than 2 fluid HP) AC conduction pumps. Efficiencies up to $43 \%$ have been obtained on large AC linear induction and DC conduction pumps.

3. A mechanical-electromagnetic (helical rotor) liquid sodium pumps, rated at 1000 fluid $\mathrm{HP}$, has been constructed for circulating $\mathbf{3 0 0 0}$ GPM with an efficiency of $28 \%$. Efficiencies of $44 \%$ have been estimated for larger units of this design.

Future requirements of the nuclear power systems are estimated to include pumps of over 100,000 GPM with fluid horsepower requirements of up to $8200 \mathrm{HP}$. Based on the review of current technology the only pump types that appear to be economically and technically feasible in sizes larger than approximately 50,000 GPM are the following:

a. Free surface mechanically shaft sealed.

b. Canned rotor.

c. Canned motor (totally enclosed). 


\section{0 FUTURE SODIUM PUMP REQUIREMENTS}

\section{1 SYSTEMS DEPENDENCY}

In order to proceed with the development of pumns for use with future large reactors, it is necessary to establish the probable hydraulic requirements. The more important of these requirements are flow, head and pumping fluid temperature.

These requirements for reactor coolant pumps are essentially systems dependent and are usually established on the basis of the overall thermalhydraulic criteria of a specific reactor system in which they are to be used. In the absence of specific system designs for large sodium cooled reactor systems from which to establish pump requirements, it is useful to establish the relationship of the more important system parameters upon the pump requirements. From these relations, probable future pump requirements may be derived for any set of reasonably assigned limits of reactor system parameters.

\subsubsection{Flow}

Pump flow requirements are quite obviously related to the reactor heat load, number of coolant loops and allowable temperature rise of the coolant.

$$
\begin{array}{ll}
F & \frac{P}{C_{p} \Delta T_{r} \quad N} \\
F & \text { coolant flow } \\
P & \text { reactor thermal power } \\
C_{p} & \text { coolant heat capacity } \\
N & \text { number of coolant loops or pumps } \\
\Delta \mathrm{T}_{\mathrm{r}} & \text { coolant temperature rise }
\end{array}
$$

The number of independent coolant loops selected for a reactor system requires consideration of both reactor safety and reliability. Where reactor safety permits and continued operation at part load is not an operation requirement, the single loop plant is feasible. More frequently, continued plant operation at part load is a required operating characteristic and multiple loops are necessary. Quite apart from these requirements, the minimum number of loops for large plants will, to a large extent, be dependent upon availability of large loop components such as heat exchangers and pumps. 


\subsubsection{Flow (Cont.)}

The liquid range of sodium extends from a melting point of $208^{\circ} \mathrm{F}$ to a normal boiling point of $1620^{\circ} \mathrm{F}$. The boiling point, which is well above any temperature necessary to achieve modern steam temperatures, suggests that physical properties of the sodium will not limit the allowable temperature rise.

In practice, the coolant temperature rise selected for use in a liquid metal reactor system is a compromise between a large number of considerations including heat exchanger terminal temperatures and pinch points, allowable thermal gradients in components, core flow limitations and pumping power. The low temperature rise systems tend to minimize heat exchanger surface and thermal gradients in the reactor core and vessel at the cost of increased pumping power, larger flow systems and the difficulties inherent in mechanical design of exchangers with large terminal differences.

\subsubsection{Pump Head}

The head developed by the circulating pumps must be adequate to meet the total system pressure drop requirements consisting of the core pressure drop and the external system drop. Of these, the core pressure drop component is severly circumscribed by nuclear considerations, heat transfer requirements and flow area limits. These restraints are quite dependent upon the type of reactor system, i.e. fast or thermal and upon the fuel geometry selection, plate, rod, pin or packed bed. In general the higher core pressure drops are to be found in fast reactor concepts due to the tighter lattice requirements and higher power densities.

The pressure drop in the external system is established by the usual design considerations for piping and heat exchanger design and are relatively independent of the reactor type.

\subsubsection{Pumping Fluid Temperature}

The temperature at which the coolant enters the pump is dependent upon the reactor core inlet and outlet temperature and upon the location of the pump in the external circuit. The trend is to improve cycle efficiencies for nuclear plants by increasing steam temperatures. Steam temperatures of $10500 \mathrm{~F}$ are representative of modern efficient steam cycles and can be achieved quite readily with sodium temperatures out of the reactor at $1200^{\circ} \mathrm{F}$. For the purposes of this study $1200^{\circ} \mathrm{F}$ is the assumed maximum steady state sodium temperature. The maximum temperature of the sodium pumped then will be $1200^{\circ} \mathrm{F}$ if the pump is located in the reactor outlet (hot leg) or $1200^{\circ} \mathrm{F}$ minus the core temperature rise if located in the reactor inlet (cold leg). 


\subsection{CURRENT DESIGN CRITERIA}

A literature survey was made of previous sodium cooled reactor designs and design studies in order to establish current practice with respect to pump design requirements and related systems design criteria.

Data from the more significant studies are shown in Table 2. It should be noted that all the reactors built or building are small and that even the "advanced" studies are, with rare exceptions, less than $500 \mathrm{MWe}$.

In reviewing these designs it may be seen that the thermal reactors of the sodium graphite (SGR) type are characterized by relatively high coolant temperature rises $\left(350^{\circ}-450^{\circ} \mathrm{F}\right)$ and head requirements of less than 200 feet reflecting the relatively open core structure and low flow requirement. On the other hand, the fast reactors (FBR) are characterized by lower coolant temperature rises $\left(250^{\circ}-350^{\circ} \mathrm{F}\right)$ and higher pump head requirements,

Corraboration of these trends and extrapolation to consideration of larger reactor capabilities was obtained by discussion with major reactor designers and from responses to a questionnaire circulated that the time this study was being made. The response to the questionnaire are summarized in Table 3.

\subsection{ESTIMATE OF FUTURE PUMP HYDRAULIC REQUIREMENTS}

A range of system parameters and the resulting sodium pump requirements for future large 1000-1500 MWe reactors and associated prototypes are summarized in Table 4 . These ranges were selected after consideration of:

1. current systems criteria shown in Table 2.

2. current opinions of future requirements of major reactors design groups.

3. compatibility with size and capacity of other loop components under development.

4. preliminary review of probable pump feasibility.

The relationship between a number of system parameters and pump hydraulic requirements are graphically presented on Figure 2. The selected limiting values for the larger reactors of both fast and thermal types are indicated on the figure by appropriate shadings.

It was assumed that the minimum number of loops for a large power system is two. Past design studies have generally been based on three loops. Currently a number of reactor designers have expressed a preference for loops of 
PRIOR DESIGNS

\begin{tabular}{|c|c|c|c|c|c|c|c|c|c|c|c|c|c|}
\hline \multirow[t]{2}{*}{ PLANT } & \multicolumn{4}{|c|}{ PLANT DATA } & \multicolumn{4}{|c|}{ GOOLANT } & \multicolumn{5}{|c|}{ PUMPS } \\
\hline & $\begin{array}{c}\text { Mute } \\
\text { GROSS }\end{array}$ & MWt & Eff\% & LờPs & $T_{1 n}$ & ToUT & $T_{\text {PUMP }}$ & $\Delta_{T c}$ & LB/HR & GPM & HEAD & HP & TYPE \\
\hline $\begin{array}{l}\text { SODIUH GRAPHITE REACTORS } \\
\text { SRE }\end{array}$ & 6 & 21 & 25 & 1 & 500 & 950 & 500 & 450 & - & 1,180 & - & - & - \\
\hline$=\operatorname{MMPF}_{M A A-S R-5700}$ & 82 & 240 & 34 & 3 & 610 & 945 & 945 & 335 & $2.8 \times 10^{6}$ & 7.200 & 160 & - & c \\
\hline $\begin{array}{l}\text { HOOULAR } \\
\text { GEAP } 3334\end{array}$ & 218 & 560 & 39.0 & 4 & 800 & 1.050 & 800 & 250 & $6.3 \times 10^{6}$ & 15.000 & 203 & - & c \\
\hline $\begin{array}{l}\text { A' ADYANCED }(1960) \\
\text { MAA-SR-3829 }\end{array}$ & 270 & 606 & 44.5 & 9 & 650 & 1.200 & 650 & 550 & $1.4 \times 10^{6}$ & 3,200 & 106 & - & $E-M$ \\
\hline $\begin{array}{l}\text { AI-CURRENT TECH } \\
(1960) \\
\text { MAA-SR-4873 }\end{array}$ & 300 & 835 & 36 & 4 & 625 & 945 & 975 & 320 & $7.3 \times 10^{6}$ & 17.500 & 160 & 1.000 & C \\
\hline $\begin{array}{l}\text { AEC PROTOTYPE } \\
\text { MUCLEAR ENGINEER } \\
\text { OCTOBER 1962 }\end{array}$ & 310 & 717 & 43 & 3 & 700 & 1,100 & 1.100 & 400 & $6.8 \times 10^{6}$ & 16,900 & 160 & $=$ & c \\
\hline $\begin{array}{l}\text { HIGH PERF SGR (AI) } \\
\text { ASME G2 VI } 24\end{array}$ & 525 & 1.207 & 43.5 & 3 & 740 & 1,160 & 1,160 & 420 & $10.8 \times 10^{6}$ & 25,400 & - & - & c \\
\hline $\begin{array}{l}\text { AEC-1000 AOV. } \\
\text { MUCLEAR ENGIIEER } \\
\text { OCTOBER I I G }\end{array}$ & 1.031 & 2.290 & 45 & 4 & 720 & 1,170 & 1.170 & 450 & $13.9 \times 10^{6}$ & 35.700 & 160 & - & c \\
\hline FAST BREEDER REACTORS & & & & & & & & & & & & & \\
\hline EBR-1 & 0.24 & 1.4 & 17 & 1 & 442 & 600 & 442 & 158 & $0.12 \times 10^{6}$ & 292 & 60 & - & $\varepsilon-M$ \\
\hline - EBR-2 & 20 & 62,5 & 32 & 2 & 700 & 900 & 700 & 200 & $1.8 \times 10^{6}$ & 4.250 & 200 & - & $c$ \\
\hline $\begin{array}{l}\text { - FERMI- } \\
\text { APOA-124 }\end{array}$ & 104 & 300 & 34.5 & 3 & 550 & 800 & $\$ 50$ & 250 & $4.4 \times 10^{6}$ & 10,000 & 330 & 1,000 & c \\
\hline $\begin{array}{l}\text { PU FAST BREEDER } \\
\text { APOA-129 }\end{array}$ & 300 & 775 & 38.7 & 3 & 650 & 1,000 & 650 & 350 & $8.3 \times 10^{6}$ & 20,200 & 223 & 1.500 & c \\
\hline $\begin{array}{l}U^{23} T H- \\
\text { NOA-2I }\end{array}$ & 315 & 865 & 36.5 & 3 & 650 & 1,000 & 650 & 350 & - & - & - & - & - \\
\hline $\begin{array}{l}\text { SETTLEO } \\
\text { BELO RAOIAL } \\
\text { BKL T-250 }\end{array}$ & 359 & 881 & 40.7 & 2 & 550 & 1.200 & 550 & 650 & $7.1 \times 10^{6}$ & 16.100 & 272 & - & - \\
\hline $\begin{array}{l}\text { SETTLED } \\
\text { BKL-7IB } \\
\text { B }-250\end{array}$ & 363 & 897 & 40.5 & 1 & 550 & 1.200 & 550 & 650 & $14.4 \times 10^{6}$ & 32.600 & 474 & - & - \\
\hline $\begin{array}{l}\text { FAST CERAMIC REACTOR } \\
\text { GEAP-3271 }\end{array}$ & 517 & 1,395 & 37 & - & 785 & 1.050 & 785 & 265 & $\begin{array}{l}60 \times 10^{6} \\
(T)^{6}\end{array}$ & $(T)$ & 326 & 13.800 & - \\
\hline $\begin{array}{l}\text { FAST OXIDE BREEDER } \\
\text { LA-2733 }\end{array}$ & - & 25,000 & & - & 593 & 850 & & 257 & $7.28 \times 10^{8}$ & - & 160 & - & - \\
\hline
\end{tabular}

- constructeo

TABLE 2 
SURVEY OF FUTURE LIQUID METAL (Na Or NaK) PUMP REQUIREMENTS

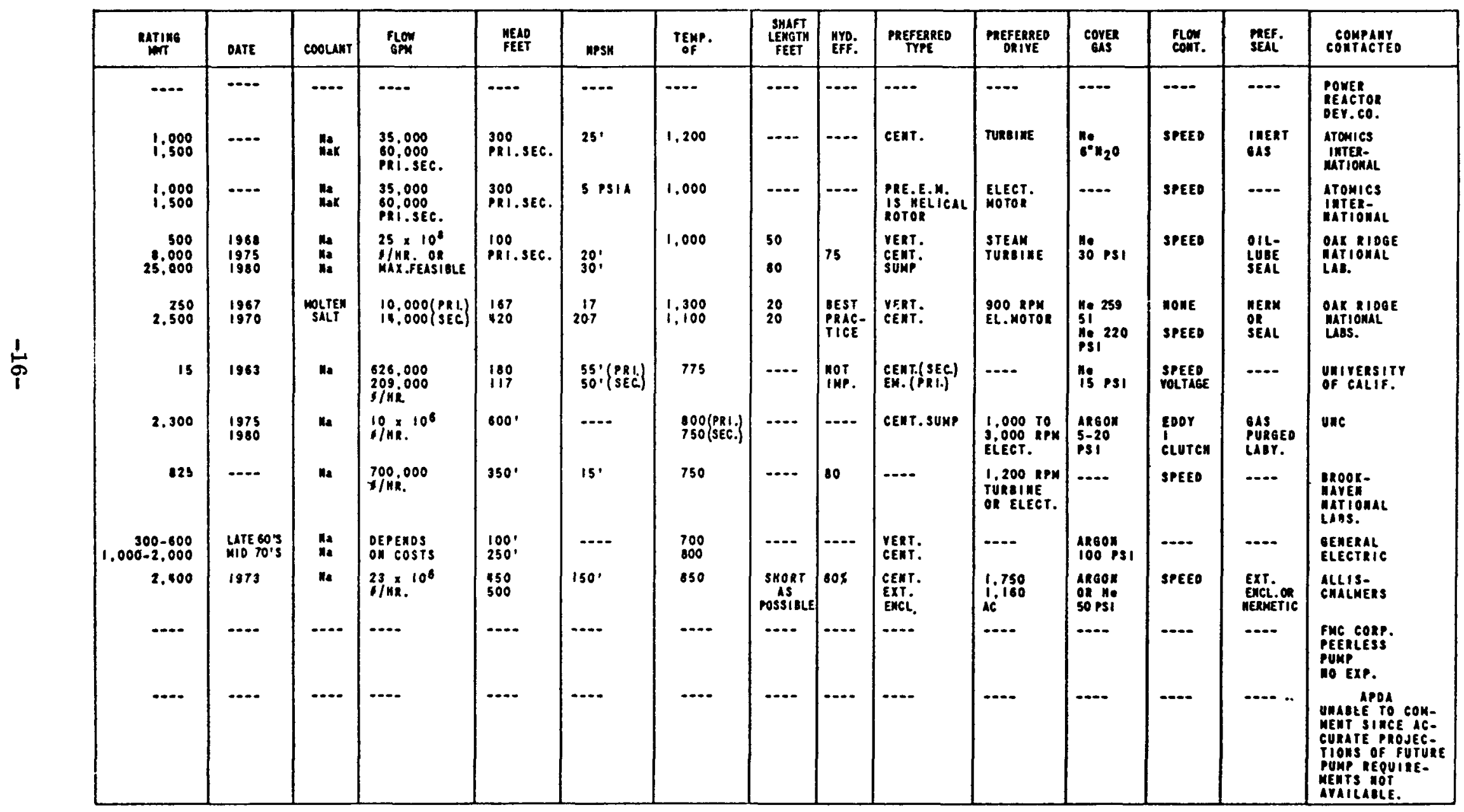

TAB LE 3 
PRIOR REQUIREMENTS

\begin{tabular}{|c|c|c|c|c|}
\hline & \multicolumn{2}{|c|}{ SODIUM GRAPHITE } & \multicolumn{2}{c|}{ FAST BREEDER } \\
\hline \multicolumn{1}{|c|}{ SYSTEM } & PROTOTYPE & FULL SIZE & PROTOTYPE & FULL SIZE \\
\hline REACTOR SIZE-MWe & $200-500$ & $1000-1500$ & $200-500$ & $1000-1500$ \\
MWT/LOOP & $300-600$ & $660-1200$ & $300-600$ & $600-1200$ \\
CORE TEMP. RISE-OF & $400-500$ & $400-500$ & $250-350$ & $250-350$ \\
PRESSURE DROP-FT.Na & $150-200$ & $150-200$ & $250-350$ & $250-350$ \\
\hline \multicolumn{1}{|c|}{ PUMP } & & & & \\
\hline FLOW- GPM X 10-3 & $15-48$ & $35-85$ & $23-63$ & $50-114$ \\
LBS/HR X 10-6 & $6.6-19$ & $15-34$ & $10-25$ & $22-46$ \\
HYDRAULIC H.P. & $500-1000$ & $1200-3600$ & $1200-4400$ & $3200-8200$ \\
\hline
\end{tabular}

TABLE 4 


\subsection{ESTIMATE OF FUTURE PUMP HYDRAULIC REQUIREMENTS (CONT.)}

approximately 250 MWe capacity, i. e. 4 loop - 1000 MWe plants. Recently, design studies have been requested for fast reactors of the $1000 \mathrm{MWe}$ size with a limitation of not more than 3 loops. It would appear that a range of $2-4$ loops is reasonable. This is equivalent to 660 to $1200 \mathrm{MWt}$ per loop for future plants with gross efficiencies in the range of $38-42 \%$. This range of sizes is also compatible with current heat exchanger development programs directed toward $1000 \mathrm{MWt}$ steam generator and sodium-to-sodium exchanger designs.

$$
3=1000 \mathrm{HWe}
$$

The selected range of temperature rise in the coolant reflects past practice. The detailed optimization of these temperatures is beyond the scope of this study and is dependent upon rather more detailed reactor system design information than is currently available for large systems at present. The result of at least one optimization study for large sodium graphite type reactors 1 seems to indicate that for conditions studied, a core temperature rise of $400^{\circ} \mathrm{F}$ is more economical than $500^{\circ} \mathrm{F}$ for a $2400 / 1050^{\circ} \mathrm{F} / 1000^{\circ} \mathrm{F}$ steam cycle.

The lower allowable temperature rise of coolant projected for the fast reactors also reflects past practice. The effect of this more conservative approach on flow requirement and pumping power is apparent in Figure 2. But even with minimum temperature rises and maximum estimated pressure drop it will be noted that the pumping power is less than $1 \%$ of the gross electric output. The economic optimization of the advantages of the more conservative design with respect to thermal gradient vs. the potential decrease size of piping, pumps, and components for greater temperature rises has not yet been developed for these systems.

(1) NAA-SR-7400, Annual Technical Progress Report, Atomics International, 1962, Page 1-B-4 

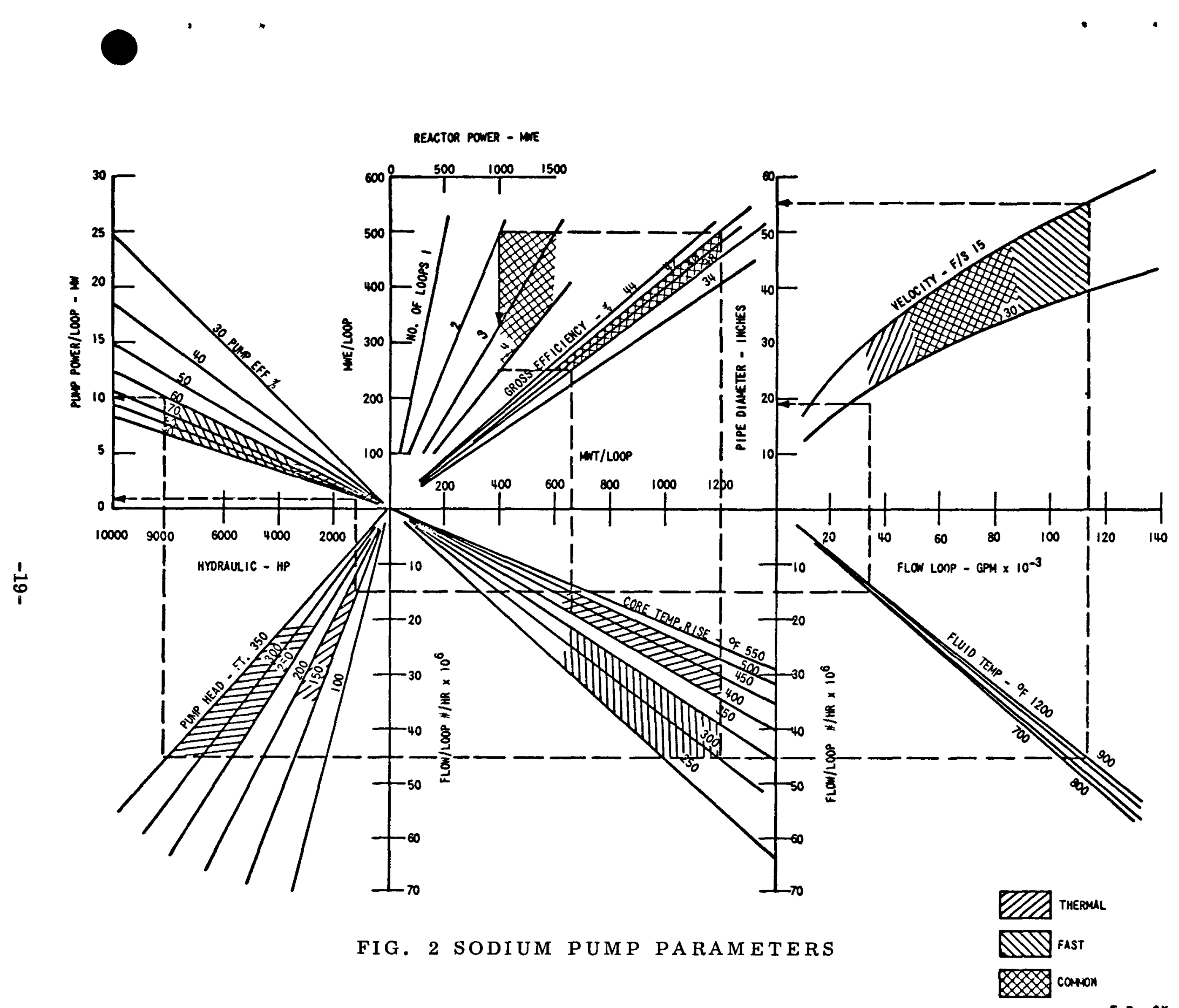

E.D. SK. 301860-B 


\subsection{FUTURE PUMP FUNCTIONAL REQUIREMENTS}

\subsubsection{General}

In addition to the functional requirements which are related to specific reactor systems considerations, there are also a number of mechanical and operational requirements which must be satisfied for practical utilization of the pumps in nuclear reactor systems. These requirements are related to problems of coolant containment, pump maintenance, flow control and layout considerations. The problems are characteristic of liquid metal cooled nuclear plant service. The same problems have been encountered and resolved in the development of the current plants. While it is anticipated that the future increases in plant size will aggravate the problems sufficiently to require substantial additional test and development, fundamentally new problems are not envisioned.

\subsubsection{Containment and Isolation of Sodium}

The extreme reactivity of the coolant and hence the danger from sodium-water reactions and from sodium-air reactions, indicates the use of either hermetically sealed systems or of systems provided with adequate non-reactive seals. The danger from leakage of the primary system sodium is increased due to its extreme radioactivity. The undesireability of contaminants in the coolant stream requires either the elimination or control of in-leakage to the coolant system.

\subsubsection{Maintenance}

Access to primary loop components of nuclear systems is generally severely limited by operations safety practice and in some cases radioactivity levels during reactor operation. Acceptable pump designs should not require routine maintenance more frequently than normal scheduled reactor shut-downs which may vary from 3 months to a year intervals. Inaccessible parts or parts that are extremely difficult to repair or replace must be designed for service life in the order of 20 years.

\subsubsection{Flow Control}

The excellent heat transfer properties of the sodium coolant make it necessary and desireable to control the coolant flow in response to power variations in such a way as to maintain reasonably constant system temperatures and thus minimize the mechanical problems of thermal shock. The required control of coolant flow can be accomplished by either varying the discharge head of the circulating pumps or by use of control valves. Both methods have been used to varying degrees in past reactor system designs. Experience to date has indicated that flow control valves are a source of trouble in sodium system and show every indication of being less desireable as flow streams 


\subsubsection{Flow Control (Cont.)}

(valve sizes) increase. The necessity of flow control by variation in pump output characteristics thus becomes correspondingly more important in the larger sizes.

\subsubsection{Layout Compatibility}

The necessary requirement to locate the primary coolant loop within a radiation shield, the mechanical limitations on pump shaft lengths, the pump attitude requirements for systems containing free surface pumps, and the desireability of access for maintenance of seals and conventional drives are all rather severe and often contradictory design limitations. 


\subsection{SELECTION OF PUMP FOR FURTHER DEVELOPMENT}

\subsection{CRITERIA}

A number of the pump types that have been developed and tested for current applications (Section 3.0) show at least some promise of meeting future requirements after sufficient additional development. Among the more promising types are the following:

\section{Mechanical}

"free surface"

shaft seal

Canned (totally

enclosed) motor

Canned rotor (dry

rotor)

Canned rotor

\section{Electro-magnetic}

AC linear induction

DC conduction

Schematic diagrams depicting the features of these pumps are shown on Figure 3.

In order to establish a practical development program, a selection from among these types is necessary. The criteria used for selection included consideration of (1) reliability, (2) cost, (3) engineering design predictability, (4) system considerations.

\subsection{RELIABILITY CONSIDERATIONS}

Reliability of any of the pump types, or any of the components of a pumping unit, is of prime concern when selecting a liquid metal pump. The components which will be considered are: pumps, drives, bearings, seals, and auxiliaries.

\subsubsection{Pump End}

For the pumps classified as "mechanical" (M), the pump end is a centrifugal pump. For the "electromagnetic" (EM) pumps, the pump end is a channel, duct or annulus. For "mechanical-electro-magnetic" (MEM) units, the pump end is an annulus or duct with a moving (mechanically rotated) rotor which directs the electro-magnetic flux lines.

It has been estimated that future full scale reactors will require up to 8,200 fluid HP/loop (based on a $1500 \mathrm{MWe}$, fast breeder reactor). The maximum fluid HP for the liquid metal pump types under consideration that have been built to date are. 


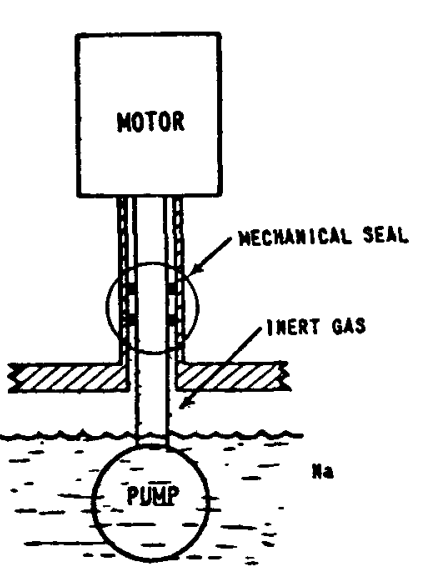

Free Surface-Shaft Seal

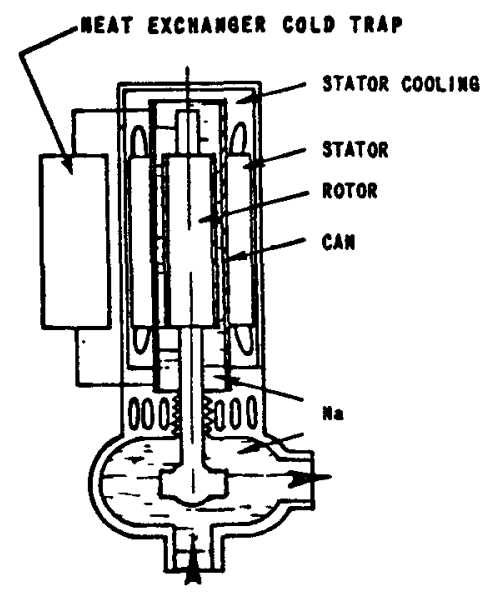

Canned Rotor

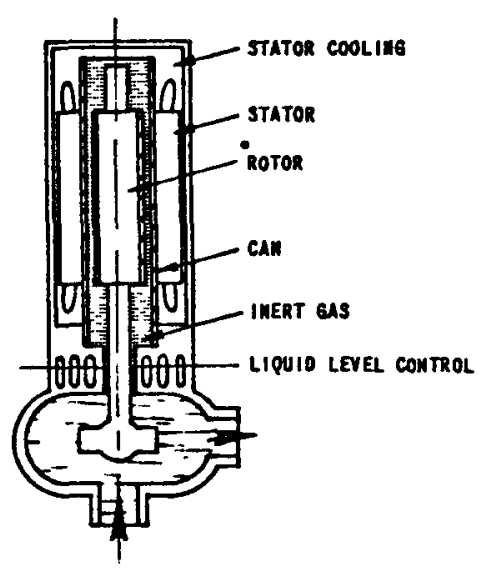

Canned Rotor (Dry Rotor)

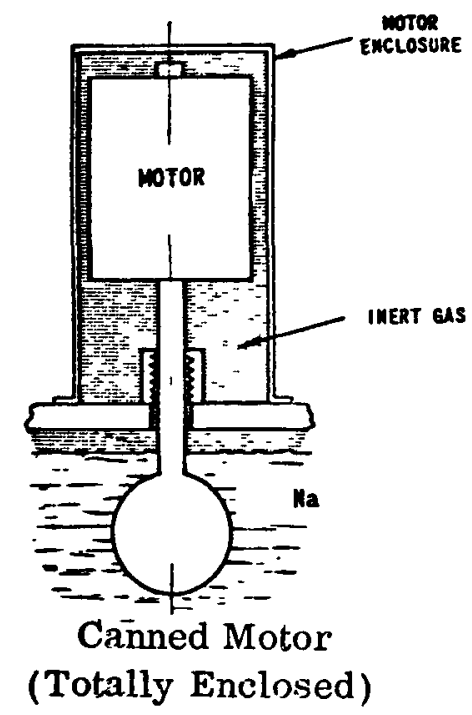

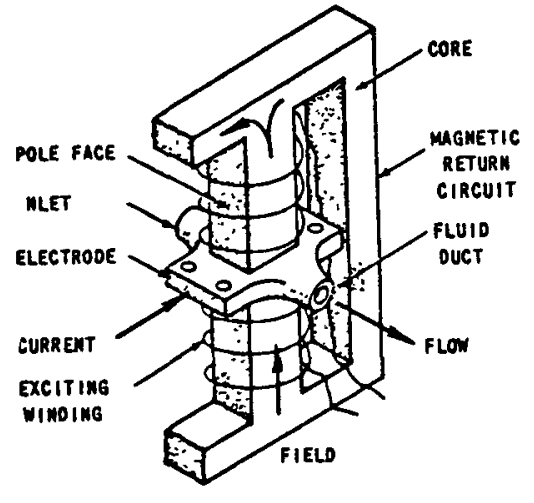

D́c Conduction

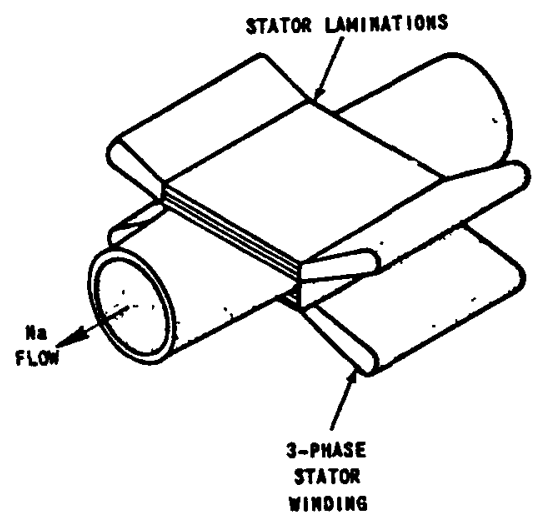

AC Linear Induction

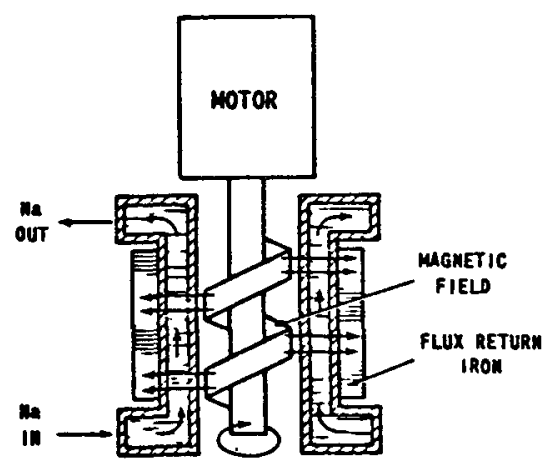

Helical Rotor

FIG. 3 FEATURES OF MECHANICAL, ELECTROMAGNETIC AND MECHANICAL 'ELECTROMAGNETIC PUMPS 


\subsubsection{Pump End (Cont.)}

Fluid HP

Mechanical Centrifugal Shaft Sealed (MCSS)

1000

Canned Rotor (CR)

750

Totally Enclosed Motor (TE)

300

AC Linear Induction (EM)

250

DC Conduction (EM)

$150 *$

Helical Rotor (MEM)

100

Canned Motor (Dry Rotor)

75

* Designed rating was 450 fluid HP, but most efficient operating rating was 150 fluid HP

Before a full scale plant or pump is constructed, a prototype will probably be built and the pump requirements will be $1200-4400$ fluid HP. While the prototype requirements are closer to the capacities of units already built, it must be assumed that the prototype pump type will be same as the eventual full size pump and the type selected must be capable of being scaled up to meet the full scale requirements.

The relatively small EM and MEM pumps now in operation, are for the most part, more reliable than mechanical pumps, but for units 20 to 50 times the capacity of the largest existing units the reliability to be expected is unknown.

The most difficult problem encountered in the development of the EM and MEM pumps has been the manufacture of the pumping ducts and annuli which must be attached to the system piping by transition sections or diffusers. Wall thickness of the ducts in the pumping sections are limited to approximately $1 / 8^{\prime \prime}$ for electrical reasons. The huge ducts or annuli which would be required to handle 50,000 -100,000 GPM would be quite delicate especially at temperatures of $1000-12000 \mathrm{~F}$. For this reason, helical rotor, induction, and conduction pumps do not appear to be very practical or reliable for high capacity applications.

Centrifugal pumps have already been built for handling over 100,000 GPM on water applications. The pump end of a centrifugal pump therefore appears to be much more reliable than the pumping sections of large EM or MEM pumps. Properly designed hydraulics (impeller, casing and diffusers, etc.) should give at least 20 years of reliable maintenance free operation. 


\subsubsection{Drive}

Drives for $M$ or MEM pumps can be anv suitable rotating drive including electric motors, turbines, engines, etc. For EM pumps, the drives consist of conductors (coils) with electrically progressed fields (induction units) or wound cores and current conductors in the case of $\mathrm{AC}$ and DC conduction types.

The drives used for most $M$ and MEM liquid metal pumps have been conventional electric motors. The majority of EM pumps have used the DC conduction or AC induction driving principle. For the size of the units built thus far, these drives have proved to be very reliable. However, for ratings of 5, 000-10,000 HP, only motor or turbine drives have "proven reliability". While theory allows the projection of EM designs to these ratings, they have not been proven and it is doubtful that EM drive reliability will ever surpass that of conventional electric motors or turbines.

Canned rotor motors with ratings of $2000 \mathrm{HP}$ have already been built for water applications, and electrically, their reljability is about equal to that of conventional motors. For liquid metal applications the highest rating for a canned rotor pump motor built thus far has been $750 \mathrm{HP}$. In order to project the canned rotor design above $1000 \mathrm{HP}$, can and bearing development work will be necessary.

Large turbine prime movers would be as reliable as large motors from a drive standpoint but containment problems for gas turbines, and the sodium-water reaction hazard for steam turbines reduces overall system reliability.

Considering all of the above, the most reliable type of drive for a large sodium pump is a conventional electric motor. The only pump types being considered for this application capable of being driven by conventional electric motors are:

1. Mechanical centrifugal shaft sealed

2. Totally enclosed canned motors

3. Helical rotor

The totally enclosed motors can use conventional AC induction motors, but unless some method of preventing sodium vapors from entering the motor are adopted, the life of the motor winding insulation is questionable. EBR II prototype testing did not reveal any noticeable attack by the sodium vapor or oxide dust after several thousand hours of operation, but until a few years of operation are accumulated on the EBR II primary pumps the reliability or life of the motor insulation will be questionable. 
5.2.2 Drive (Cont.)

This leaves the mechanical centrifugal shaft sealed and helical rotor as the only two types that can be driven by conventional motors with proven reliability.

\subsubsection{Bearings}

EM pumps ends do not have bearings because there are no moving parts. MEM pump ends do not have any bearings contacted by the liquid metal but the rotor of the pump end requires conventional radial bearings to support the rotor shaft unless an over-hung shaft design is used.

The pump ends of mechanical units require radial bearings in the liquid metal unless an over-hung shaft design is used.

The EM and MEM pumps do not have bearings operating in the liquid metal, so naturally, the question of sodium bearing reliability does not exist for EM and MEM pump types.

The reliability of liquid metal lubricated bearings for $M$ pumps is still questionable. The EFAPP and HNPF hydrostatic bearings, and the thermoflex hydrodynamic bearings on the CANEL canned rotor pumps have performed satisfactorily thus far, but until more operational hours are accumulated they cannot be labeled as completely reliable. Bearings for 5,000 - 10,000 HP mechanical pumps can be projected from the existing smaller units but until full scale development testing is performed reliability will remain questionable.

Because of the efficiency advantage of $M$ pumps and the areas of doubt of the reliability of large EM and MEM ducts, the direction of development efforts toward reliable liquid metal lubricated bearings is necessary. EM and MEM pumps have a definite advantage in the bearing reliability area but expected successful development of large sodium bearings could erase this advantage.

\section{2 .4 Seals}

The seals for EM and MEM and canned rotor mechanical pumps are the ducts, annuli or cans which contain the liquid metal, and since these barriers are static enclosures more than seals, they are usually called "seal-less" or hermetically sealed pumps. The mechanical centrifugal shaft. sealed (MCSS) pumps require some type of shaft seal to contain the liquid metal, or in the case of free surface to sump pumps, a seal to contain the cover gas.

The stationary metallic barriers (cans, ducts, annuli) used in conjunction with EM and MEM pumps are the major cause of the low efficiencies of these types. Non-metallic barriers would improve the efficiency but methods for joining and sealing non-metals (ceramics) for high temperature sodium applications have not been developed. 


\subsubsection{Seals (Cont.)}

As mentioned in the section on pump ends, the thin cans and ducts used on EM and MEM pumps present strength and thermal design difficulties which are amplified as the size or capacity of the units is increased. For this reason (and the drive and efficiency advantages of mechanical centrifugal pumps) shaft sealing appears to be the best approach for large units.

The most practical and reliable shaft sealing technique used on stationary sodium systems has been the free surface, gas blanketed approach. With this method, gravity keeps the liquid sodium away from the shaft seal area and the only requirement of the seal is to contain the cover gas.

The HNPF and EFAPP pumps use face type shaft seals to contain the low pressure (1/2 psi) cover gas. The EBR II primary pumps are totally enclosed types, but all three plants use the free surface technique. As mentioned in Section 5.2.2, the reliability of unprotected motor insulation in the TE pumps is questionable. So at the present time, the best seal approach appears to be a rotating shaft seal for the containment of low pressure argon or helium gas.

No shaft seal problems have been reported at the HNPF as yet, but excessive seal leakage (oil leakage) has been a problem at EFAPP. Both plants are using oil lubricated rubbing seals, but the EFAPP primary pumps are using a non-hydrogenous oil (fluorolube) which does not have as good lubricating properties as the mineral oil used at Hallam.

While the various types of shaft seals have been fairly reliable on many gas sealing applications, they have never been fully developed for sodium-nuclear applications. It would appear that a shaft seal especially designed for a large free surface sodium pump could be developed to perform more reliably and with less maintenance than presently used sodium pump seals. The type of seal used on large hydrogen cooled generators has "proven reliability", and this principle seems practical for sodium pump applications. Other types also have possibilities.

Assuming that reliable seals and bearings can be developed, the mechanical centrifugal shaft sealed pump will have better mechanical reliability than any other pump type.

\subsubsection{Auxiliaries}

Auxiliaries for liquid metal pumps include all apparatus necessary for cooling, lubricating, controlling flow, and supplying the power for their operation (not including standard $\mathrm{AC}$ power). The required auxiliaries for the various pump units are listed below. 


\subsubsection{Auxiliaries (Cont.)}

1. Mechanical Centrifugal Oil Lubricated Shaft Sealed

(a) Lube oil system for motor bearings unless anti-friction bearings are used.

(b) Lube oil system for shaft seal

(c) Variable frequency power supply or rheostats if wound rotor motors are used.

2. Mechanical Centrifugal Gas Lubricated Shaft Sealed

(a) Lube oil system for motor bearings unless anti-friction bearings are used.

(b) Inert gas supply to lubricate sea! and to purge seal area of sodium vapors

(c) Variable frequency power supply or rheostats if wound rotor motors are used.

3. Totally Enclosed Canned Motor

(a) Inert gas supply to gas filled motor enclosure

(b) Cooling system to remove motor losses

(c) Lube oill system for motor bearings unless anti-friction bearings are used

(d) Variable frequency power supply for flow (speed) control

4. Canned Rotor (Enclosed Dry Rotor)

(a) Inert gas supply to rotor area

(b) Motor cooling system (water, oil, gas)

(c) Lubricating system for motor bearings unless anti-friction bearings are used

(d) Variable frequency power supply 


\subsubsection{Auxiliaries (Cont.)}

\section{Canned Rotor}

(a) Cooling system to remove motor losses

(b) Cold trap to remove oxides from motor end

(c) Variable frequency power supply

6. AC Linear Induction - AC linear induction pumps requiring no auxiliaries other than voltage control have been successfully operated. However, for large units, some cooling will probably be necessary.

7. DC Conduction - No auxiliaries are required for the pumping section, but the following would be needed for the motor driven homo-polar generator:

(a) /Lube oil system for motor and generator bearings, unless anti friction bearings are used.

(b) Water cooling for generator

8. Helical Rotor

(a) Air or gas cooling for helical rotor windings

(b) Lube oil supply for drive motor bearings, unless antifriction bearings are used

(c) DC power supply for pump end rotor

The mechanical-centrifugal, oil lubricated shaft sealed, pump would not require any non-conventional auxiliary systems. The only auxiliary systems necessary in the reactor compartment would be the oil service systems for the motor bearings and shaft seal. The systems would be located above the floor level and would be easily accessible for inspection and maintenance. For these reasons, this type of pumping unit appears to have the most reliable auxiliary systems.

\section{$5.3 \cos \mathrm{T}$}

In comparing pump costs, it is necessary to consider both the capital and the operating costs. The initial or capital costs for this comparison include the dollars which would be paid for the pumps and pump drives (including auxiliaries) 


\section{$5.3 \operatorname{cosT}$ (CONT.)}

required for a $1500 \mathrm{MWe}$ sodium cooled reactor plant after a prototype had been developed and tested. The operating costs include the maintenance costs, and the cost of energy consumed by the pumps, drives, and auxiliaries.

\subsubsection{Operating Costs}

An estimated approximate twenty year energy cost comparison for the various pump types, based on the maximum estimated efficiencies, is shown in Table 5. The plot of overall pump efficiency vs. 20 year energy cost shown in Figure 4 is based on estimated fluid HP requirements of:

$$
\begin{aligned}
& \text { Primary }-15,000 \text { fluid } \mathrm{HP}=11,200 \mathrm{KW} \\
& \text { Secondary }-7,500 \text { fluid HP }=5,600 \mathrm{KW}
\end{aligned}
$$

From the curves of Figure 4 , a plant with $80 \%$ efficient pumps will cost 15 million dollars less to operate than one with $45 \%$ efficient pumps over a 20 year period. The mechanical centrifugal shaft seal and totally enclosed pumps can be designed with overall efficiencies over $80 \%$, and from a power cost standpoint, they are far superior to any of the other pump types available. In order to obtain speed (flow) control, a variable frequency power supply is required for the totally enclosed design (squirrel cage motor) whereas the shaft seal design could be driven by a wound rotor motor. The wound rotor motor drive would result in a $2-3 \%$ efficiency advantage for the shaft seal approach over the totally enclosed design.

The maintenance cost for units over $1000 \mathrm{HP}$ are unknown, at the present time, but some estimations can be made based on past performance of smaller existing units. At the present time, EM pumps require the least maintenance of any of the pump types. The MEM (Helical Rotor) types do not contain any seals or wear parts other than conventional bearings and the maintenance requirements are quite low. The totally enclosed types do not contain seals and if bearing operation is satisfactory, the only possible maintenance problem may be the replacement of deteriorated motor windings after several years of operation.

Shaft sealed units contain both bearings and seals, and at the present time, bearings have not required any maintenance (EFAPP and HNPF), but the seals do require periodic maintenance.

Canned rotor units appear virtually maintenance free with the exception of cold trap draining requirements, but not enough hours have been accumulated as yet to make any definite statements. 
20 YEAR ENERGY COSTS

\begin{tabular}{|c|c|c|c|c|}
\hline PUMP TYPE & $\underset{\%}{\mathrm{EFF}}$ & $\begin{array}{c}\text { DOLLARS FOR } 20 \\
\text { PRIMARY }\end{array}$ & $\begin{array}{l}\text { RS OPERATION* } \\
\text { SECONDARY }\end{array}$ & TOTAL \\
\hline $\begin{array}{l}\text { MECH-CENT. SHAFT } \\
\text { SEAL }\end{array}$ & 83 & $\$ 11,800,000$ & $\$ 5,900,000$ & $\$ 17,700,000$ \\
\hline TOTALLY ENC.MOTOR & 80 & $12,250,000$ & $6,125,100$ & $18,375,000$ \\
\hline CANNED (DRY) ROTOR & 65 & $15,100,000$ & $7,550,000$ & $22,650,000$ \\
\hline CANMED ROTOR & 60 & $16,300,000$ & $8,150,000$ & $24,450,000$ \\
\hline AC LINEAR & 50 & $19,600,000$ & $9,800,000$ & $29,400,000$ \\
\hline DC CONDUCTION & 50 & $19,600,000$ & $9,800,000$ & $29,400,000$ \\
\hline HELICAL ROTOR & 45 & $21,800,000$ & $11,900,000$ & $33,700,000$ \\
\hline
\end{tabular}

*POWER COST BASED ON .005 $\$ / \mathrm{KW}$. HR.

PRI. 15,000 FLU10 HP

SEC. 7,500 FLUID HP

TABLE 5 


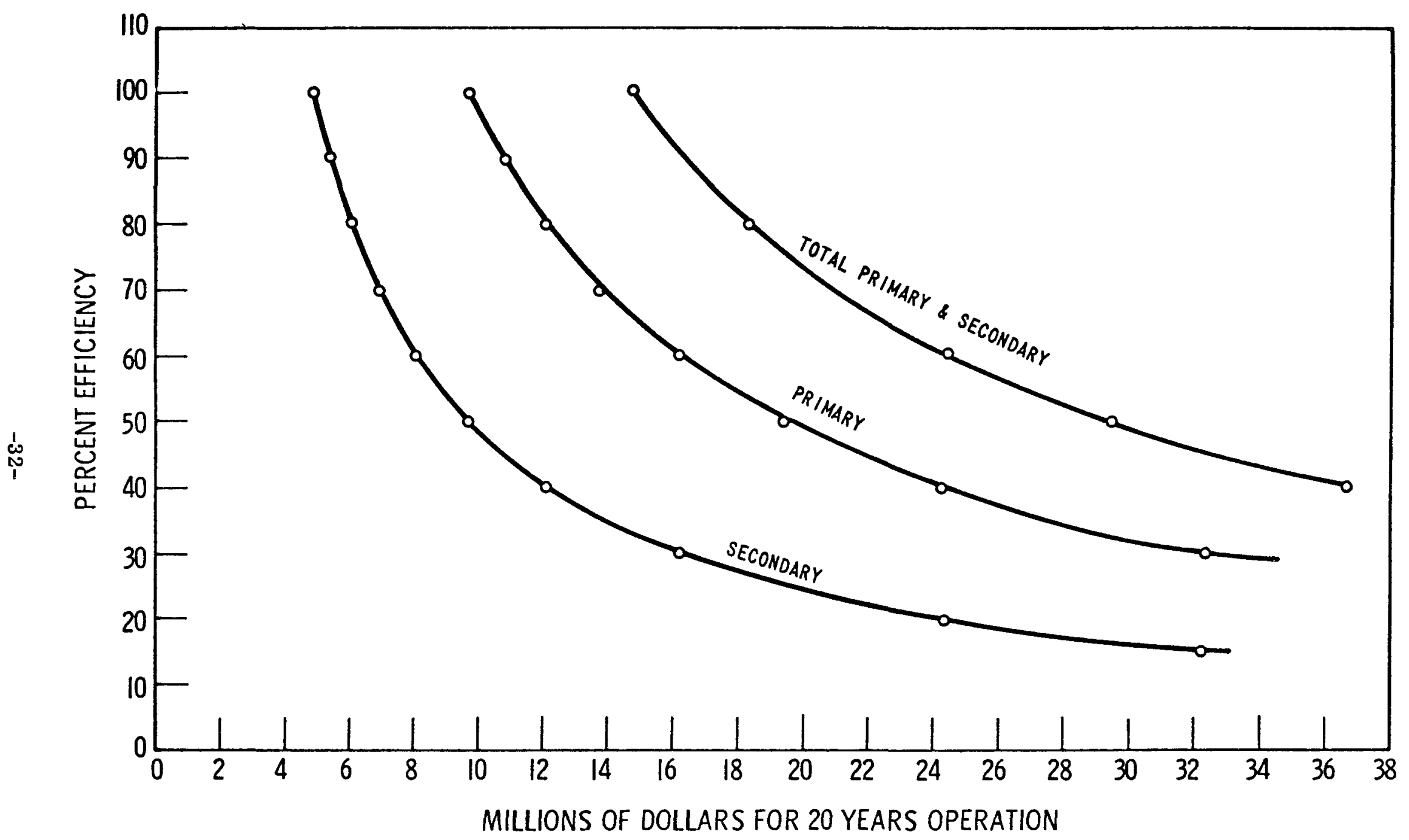

FIG. 4 OVERALL PUMP EFFICIENCY VS. 20 YEAR POWER COST

E. D. SK. 301856-B 


\subsubsection{Operating Costs (Cont.)}

If a unit must be removed from the sodium system for maintenance or repairs to the pump end, EM pumps with removable coils or helical rotor pumps have a definite advantage. Either type can be removed without opening the sodium system. However, the possibility of duct repair due to erosion may be more of a problem with these types than electrical or drive repairs, and this would involve plant shut down and major maintenance work.

The mechanical centrifugal pump ends should not require any maintenance for 10-20 years (depending on the operation of the lower radial bearings usually located near the pump end). The only item requiring periodic maintenance, at the present time on the HNPF and EFAPP pumps, are the shaft seals and speed control apparatus.

Improved seal design should reduce, if not eliminate seal maintenance, and speed control systems (liquid rheostats or Ward-Leonard systems, etc.) can also be improved so as not to be a major maintenance item.

The bearing and seal performance for large sodium pumps will only be answered by developmental testing and final proof testing, but at the present time successful results are expected. Since the MCSS units offer other advantages in both cost and reliability, the development required can be justified. Even if maintenance costs remain higher with shaft sealed pumps, the savings in energy costs will probably outweight the difference, and the MCSS units have to be rated "best" from an operating cost standpoint.

\subsubsection{Initial Costs}

Since liquid metal pumps of this size have never been constructed, manufacturing cost estimates are difficult to make. The EFAPP and HNPF mechanical centrifugal shaft sealed pumps cost approximately $\$ 300$ per fluid HP. Based on this, a 5,000 fluid HP MCSS pump would cost slightly less than $\$ 1,500,000$. The cost of a 5,000 fluid HP totally enclosed pump would be about the same, since both types are about the same physical size, and the only major difference in cost would be the required seals and cooling systems.

The helical rotor design would require a drive motor rated $40 \%$ higher than the motor required for the $80 \%$ efficient MCSS or TE pumps. This would increase the cost of the drive and it is doubtful whether the helical rotor pump end would cost less than a mechanical-centrifugal pump end. Capital costs then, for canned motor, canned rotor, and helical rotor pumps, would be more than MCSS or TE pumps.

The cost of the motor driven homo-polar generator and DC conduction pump would be considerably more than a MCSS or TE pump. 


\subsubsection{Initial Cost (Cont.)}

The weight of a 115 fluid HP AC linear induction pump is approximately 8,000 lbs. A 5,000 fluid HP unit then may weight as much as $300,000-400,000 \mathrm{lbs}$. and the cost of the copper for the windings could cost $\$ 1,000,000$. Therefore, it is doubtful if an AC linear pump could be built any cheaper than any of the other pump types.

In summary, MCSS and TE pumps would be the least expensive, and the DC conduction type would be the most expensive, with a $1 / 2$ million dollar per primary pump spread between the two types (5000 HP units). The initial costs of the other four types would fall somewhere in between.

\subsection{DESIGN PREDICTABILITY}

None of the pump types under consideration have been built in sizes that are comparable to those indicated for future $1500 \mathrm{MWe}$ sodium cooled plants. In order to scale up any of the existing types, some development and testing will be necessary. However, much of the design theory used for motor driven centrifugal water pumps can be applied to liquid metal pump design and electrically and hydraulically these designs would be quite predictable. EM pump designs for small units are predictable, but for high capacity pumps the magnetic design for the large magnetic gaps becomes very unpredictable as pointed out with the DC EM prototype pump tested for the EBR II application. Unexpected flux leakage in this design caused actual output to be 50-75\% less than predicted output.

AC linear and helical rotor EM and MEM pumps have been predictable in small (less than 10,000 GPM) sized units. In extrapolating the design to 50,000-100,000 GPM capacities electrical (magnetic), the large flux gaps make the design unpredictable and cooling and fabricating techniques would have to be tried and tested before any degree of overall design predictability can be assured. While both of these types appear feasible, considerable development would be necessary, and since efficiency is less and reliability is unknown, this development cannot be justified.

Canned rotor pumps would have predictable hydraulics, but "can" and insulation development would be needed and efficiency still would not be as good as uncanned mechanical pumps. This development cannot be justified at this time, unless the free surface system cannot be used.

The centrifugal pump types which can be driven by conventional electric motors with conventional bearings are the totally enclosed motor, and shaft sealed units. Electrical and hydraulical characteristics of both types are very similar from an engineering design standpoint. The mechanical designs are also similar except for bearing and seal design. Therefore, the relative design predictability is dependent on the mechanical consideration of bearing and seal requirements. 


\subsection{DESIGN PREDICTABILITY (CONT.)}

The totally enclosed type does not have a shaft seal, so the question of leakage rate predictability is nearly eliminated. Since the motor insulation life data is not available, the motor life cannot be predicted. Also, motor cooling and purging system design could also create problems.

The fluid seal design for a shaft seal pump could be based on extensive design experience with hydrogen cooled turbo-generator shaft seals. Excellent results would be expected, and while some development work on materials, fluid selection, and mechanical layout is necessary, the design approach would give a maximum degree of engineering design predictability in regard to life, reliability, and leak tightness.

Both of these types require a radial bearing to support the shaft near the pump end. While large diameter liquid metal bearings will require development, bearing operation (hydrostatic) on the EFAPP and HNPF pumps appear good, and this approach is feasible for larger units. Development testing could provide the analytical tools necessary for the design of both hydrostatic and hydrodynamic bearings for high capacity sodium pumping applications.

\subsection{SYSTEM CONSIDERATIONS}

\subsubsection{Layout}

Figure 5 shows the eight basic layout schemes for reactor primary loops. The direction of coolant flow can be either "up" or "down". The coolant circulating pumps can be placed before (hot leg) or after (cold leg) the heat exchanger, with respect to the direction of flow. The pumps can be located either "high" or "low" with respect to the reactor vessel.

Reactor "down-flow" is desirable since it would ease the requirements on hold down mechanisms. But, if a free surface is to be maintained at the top of the reactor vessel (for sealing reasons), the gas head on the free surface must be greater than the pressure drop across the reactor, or the free surface would be lost. The pressure drops across future sodium cooled reactors may be as high as 100-150 psi. (EFAPP reactor $\triangle \mathrm{P}=74 \mathrm{psi}$ ).

With reactor "up flow", the pressure of the gas head on the reactor must approximate the pressure drop across the heat exchanger. Since this pressure drop is usually low (5-10 psi) "up flow" is more frequently used because of the resulting reduced reactor pressure sealing requirements.

From a thermal pump design standpoint, placement of the pump in the cold leg is desirable since design temperatures are only $600^{\circ} \mathrm{F}$ compared to $1200^{\circ} \mathrm{F}$, if placed in the hot leg. 


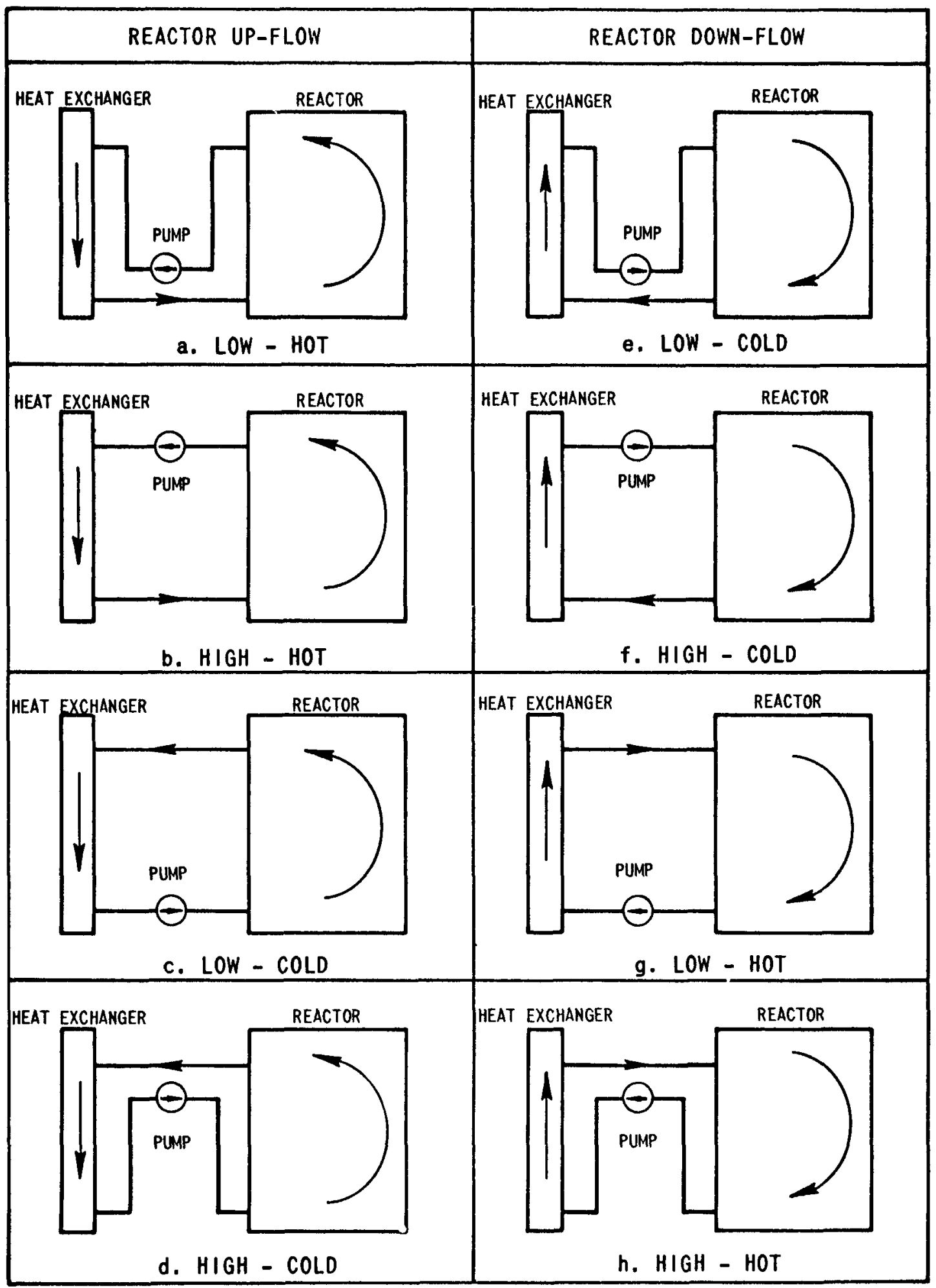

FIG. 5 BASIC PRIMARY LOOP ARRANGEMENTS

E.D. SK. 301857 


\subsubsection{Layout (Cont.)}

The "low" arrangements result in increased available pump suction head due to the added static head of the sodium. The "high" pump location allows the use of a shorter pump shaft (free surface pumps).

From hydraulic standpoint, the best layout scheme would be the "low-coldup flow" arrangement, if the free surface pump approach is taken. However, the long shaft required for the "low design" would impose bearing and shafting problems and the "high-cold-up flow" approach is probably more practical.

If hermetic pumps are used, they can be located low or high with respect to the reactor without imposing long shaft or piping problems. With a completely hermetic system (including hermetic control rods), the system could be filled and pressurized and smaller high speed units could be used for either up or down flow. So, while hermetically sealed pumps and control rods are more expensive (initial and operating) and require more development than free surface designs, they would be more versatile from a layout standpoint.

While available NPSH is increased with the "low" arrangements, high capacity sodium pumps with low NPSH requirements can be developed. The few added feet of static height to be gained by locating the pump end low would not justify the added shaft length and associated dynamic design problems. The fluid sealed or totally enclosed approaches allow some cover gas pressurization (30-50 psi) which is equal to $82-137$ feet of 10000F sodium. A summary of the layout arrangement considerations is shown in Table 6.

The "high-hot-up flow" scheme appears to be the best approach from a reactor and piping layout standpoint. While "high-cold-up flow" would create less pump thermal design problems it is felt that pump operating temperatures of $1200^{\circ} \mathrm{F}$ for the free surface designs are feasible.

\subsubsection{Elow Control}

The degree of flow control required for a sodium cooled reactor power plant depends largerly on the design of the reactor core and the heat exchangers. In order to balance heat input and output into the coolant loops some flow control is necessary. It is anticipated that at least ( $3: 1$ flow control) will be necessary during startup or for operation at reduced power levels for future (1500 MWe) plants.

At the present time, speed control (mechanical-centrifugal pumps) and voltage or current control (EM and MEM pumps) are the most commonly used methods of controlling flow. Throttle valves have not proved to be reliable as yet in sodium systems. 
PUMP - REACTOR ARRANGEMENT SUMMARY

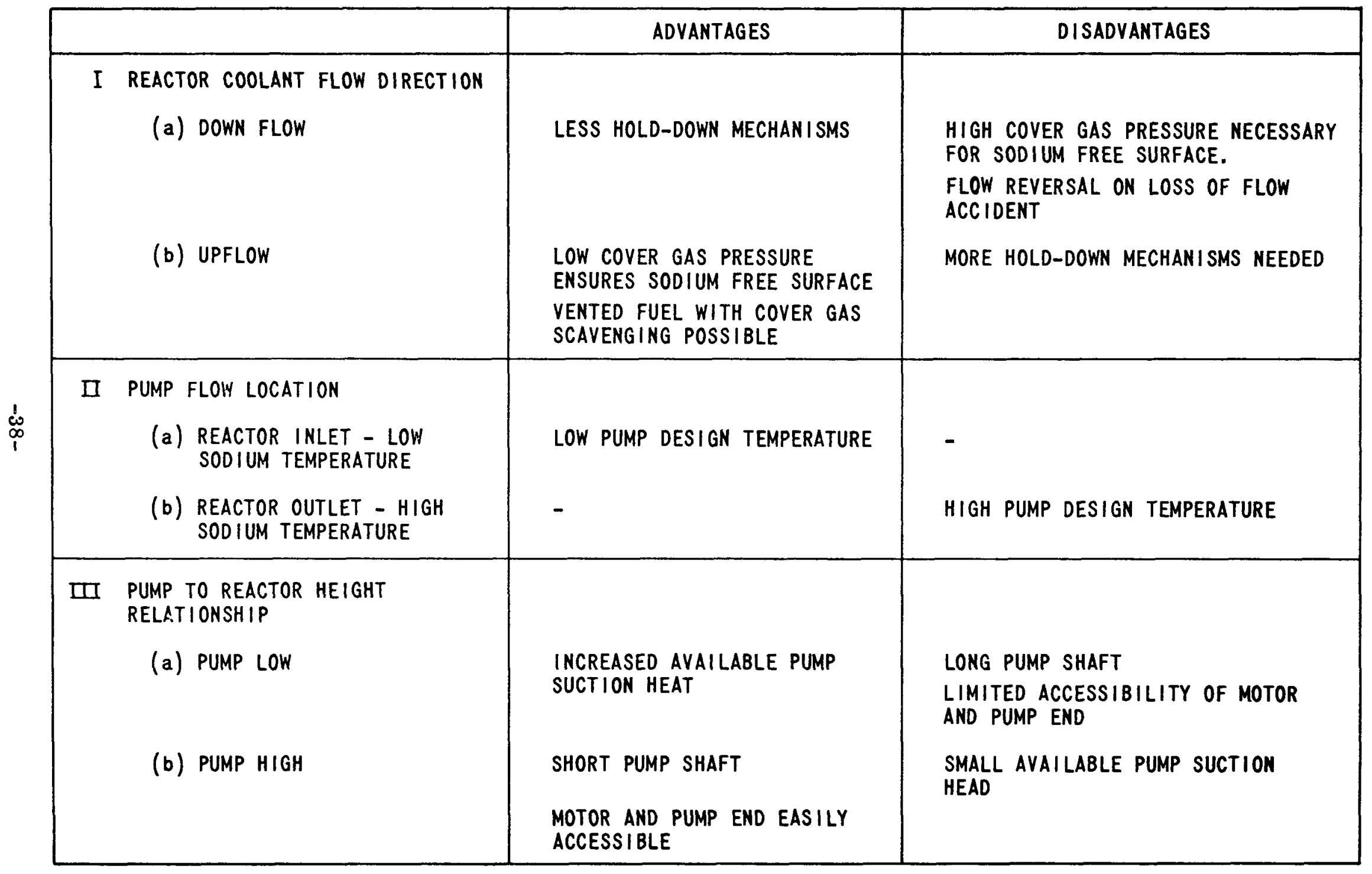

TABLE 6 


\subsubsection{Flow Control (Cont.)}

The required equipment for EM and MEM pump flow control is relatively simple and inexpensive as compared to $\mathrm{MG}$ sets, eddy current couplings or wound rotor motors usually used in conjunction with $\mathrm{AC}$ motor driven mechanical centrifugal pumps. However, the centrifugal pump offers so many other advantages over EM and MEM pumps (size, cost, reliability, etc.) that the disadvantage of requiring auxiliary equipment for speed control is usually outweighed when considering high capacity requirements.

A detailed study would have to be made after more of the operating requirements are determined in order to select the best type of variable speed drive. However, $5000-10,000 \mathrm{HP}$ wound rotor motors (4:1 speed range) with efficiencies of over 95\% are available now and this appears to be the best direction to take for this application at the present time.

Figure 6 shows some of the possible variable speed drive arrangements with associated efficiency estimates. The wound rotor motor drive of $95 \%$ is the highest followed by the squirrel cage motor - eddy current coupling drive with an efficiency of $90 \%$. Both of these drives are suitable for shaft seal approaches only. The best drive efficiency estimated for a totally enclosed variable speed drive is $85 \%$.

\subsubsection{Shielding}

The shaft sealed, totally enclosed, and enclosed rotor pumps are usually vertical designs that allow location of the drive end above a shielded floor. This is in contrast to the totally enclosed and enclosed rotor designs where no means ( other than baffling or purging ) are available to prevent sodium vapor or other containments from entering the motor or rotor cavity.

The helical rotor, AC induction and canned rotor types contain electrical windings in close proximity to the sodium and require special heat and radiation resistant insulation.

The mechanical centrifugal shaft sealed pump is then the only pump type that mechanically divorces the drive end from the pump end and does not utilize electrical windings below the shield or floor level.

\section{5 .4 Safety}

All EM and MEM pumps inherently require the use of thin walled ducts or annuli. On high capacity designs these ducts will be quite delicate, and the danger of sodium leakage will be a critical safety problem.

The casings of centrifugal pumps can be made to any desired wall thickness without sacrificing efficiency and the danger of a sodium leak developing in the pump end is remote. 


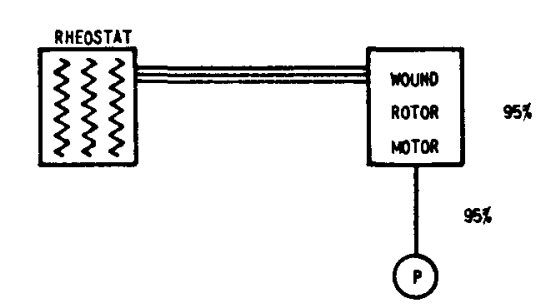

(a) AC WOUND ROTOR MOTOR

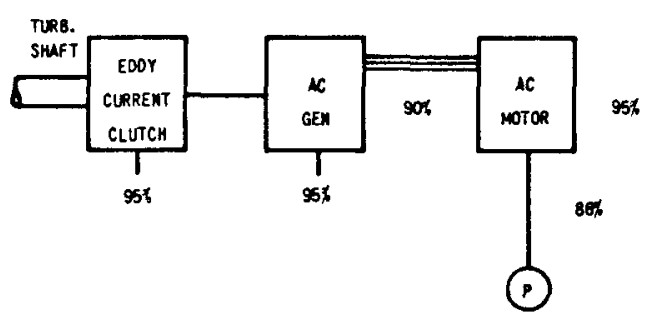

(d) MAIM TURBIME-EDOY CURRENT CLUTCH YARIABLE FREQUENCY.

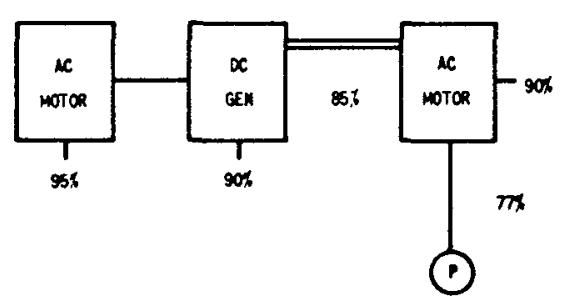

(g) OC MOTOR DRIVE

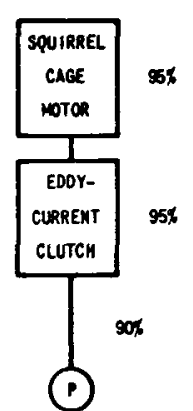

(b) EDOY CURRENT COUPLINGS

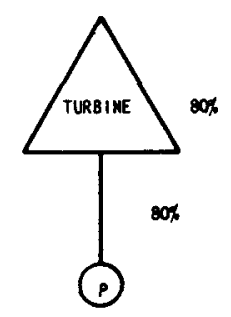

(a) DIRECT TURBINE DRIVE

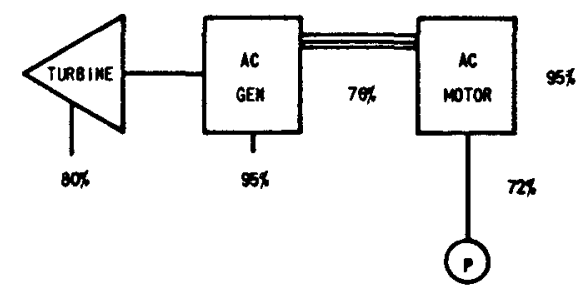

(h) TURBINE - GEMERATOR - VARIABLE FREQUEMCY

FIG. 6 DRIVE EFFICIENCIES

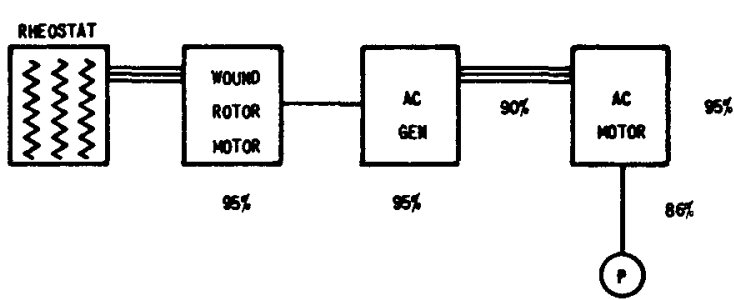

(c) WOUND ROTOR CONTROLLED YARIABLE FREQUENCY

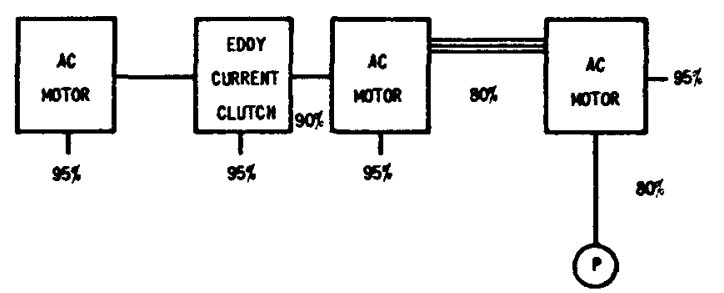

(f) AC MOTOR - EDOY CURRENT CLUTCH - VARIABLE FREQUEMCY

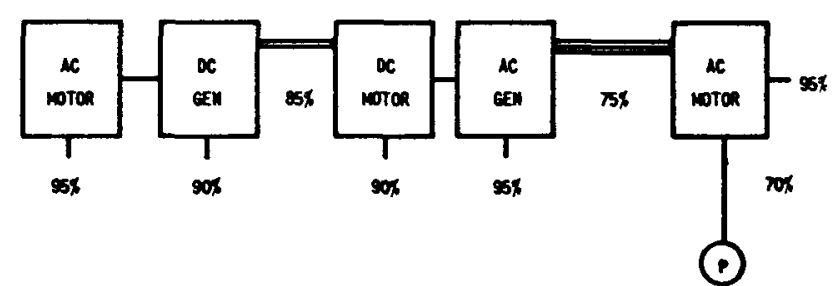

(i) AC-DC IC YARIABLE FREQUENCY

E.D. SK.301859-8 


\subsubsection{Safety (Cont.)}

Canned rotor pumps would have a secondary barrier to contain sodium if a leak developed in the stator can, since these units are usually enclosed in an outer shell. However, a can leak would completely destroy the stator and costly major repairs would be required.

The free surface centrifugal approach appears to be the safest scheme, in that failure of any of the components (bearings, seals or drive) would not create any sodium leakage or radiation hazard.

\section{6 SELECTION OF TYPE FOR FUTURE DEVELOPMENT}

A comparative rating of the various pump types is presented in Table 7 . The ratings are based on present estimations for the construction of these types with capacities in excess of 50,000 GPM and horsepower ratings over $3000 \mathrm{HP}$.

The centrifugal type pump is the only pump that is recommended for development at this time to meet the future large pump requirements. Programs are presented in Sections 7, 8, \& 9 for the development of the two most promising types, (1) mechanical centrifugal with shaft seal and (2) mechanical centrifugal with totally enclosed motor.

In preparing Table 7 it was recognized that the "reliability" and "predictability" ratings for electromagnetic and mechanical-electromagnetic pumps are considerably better for small pumps, i. e. under 10,000 GPM or 1000 fluid HP than is indicated in Table 7. This is due to simpler duct fabrication and magnetic design problems in small sizes. On this basis electromagnetic and mechanical-electromagnetic pumps might show promise for use for prototype reactor use. The choice of mechanical centrifugal pumps for further development is based on the future requirements of large pumps in the order of 100,000 GPM capacities. 
COMPARATIVE PUMP RATINGS

\begin{tabular}{|c|c|c|c|c|c|}
\hline PUMP TYPE & RELIAB ILITY & $\operatorname{cost}$ & $\begin{array}{c}\text { DESIGN } \\
\text { PREDICTABILITY }\end{array}$ & $\begin{array}{c}\text { SYSTEM } \\
\text { CONSIDERATIONS } \\
\end{array}$ & $\begin{array}{l}\text { AVERAGE } \\
\text { RATING }\end{array}$ \\
\hline $\begin{array}{l}\text { MECHANICAL CENTRIFUGAL } \\
\text { SHAFT SEALED (M) }\end{array}$ & EXCELLENT & EXCELLENT & EXCELLENT & GOOD & 3.75 \\
\hline $\begin{array}{l}\text { TOTALLY ENCLOSED CANNED } \\
\text { MOTOR } \\
\text { M) }\end{array}$ & GOOD & GOOD & EXCELLENT & FAIR & 3.0 \\
\hline CANKED ROTOR (M) & FAIR & $F A \mid R$ & GOOD & GOOD & 2.5 \\
\hline HELICAL ROTOR (MEM) & GOOD & POOR & $F A \mid R$ & GOOD & 2.25 \\
\hline $\begin{array}{l}\text { ENCLOSED ROTOR } \\
\text { CANNED MOTOR (M) }\end{array}$ & FAIR & FAIR & GOOD & FAIR & 2.25 \\
\hline AC INDUCTION (EM) & GOOD & POOR & $F A \mid R$ & $F A \mid R$ & 2.0 \\
\hline DC CONDUCTION (EM) & GOOD & POOR & POOR & FAIR & 1.75 \\
\hline
\end{tabular}




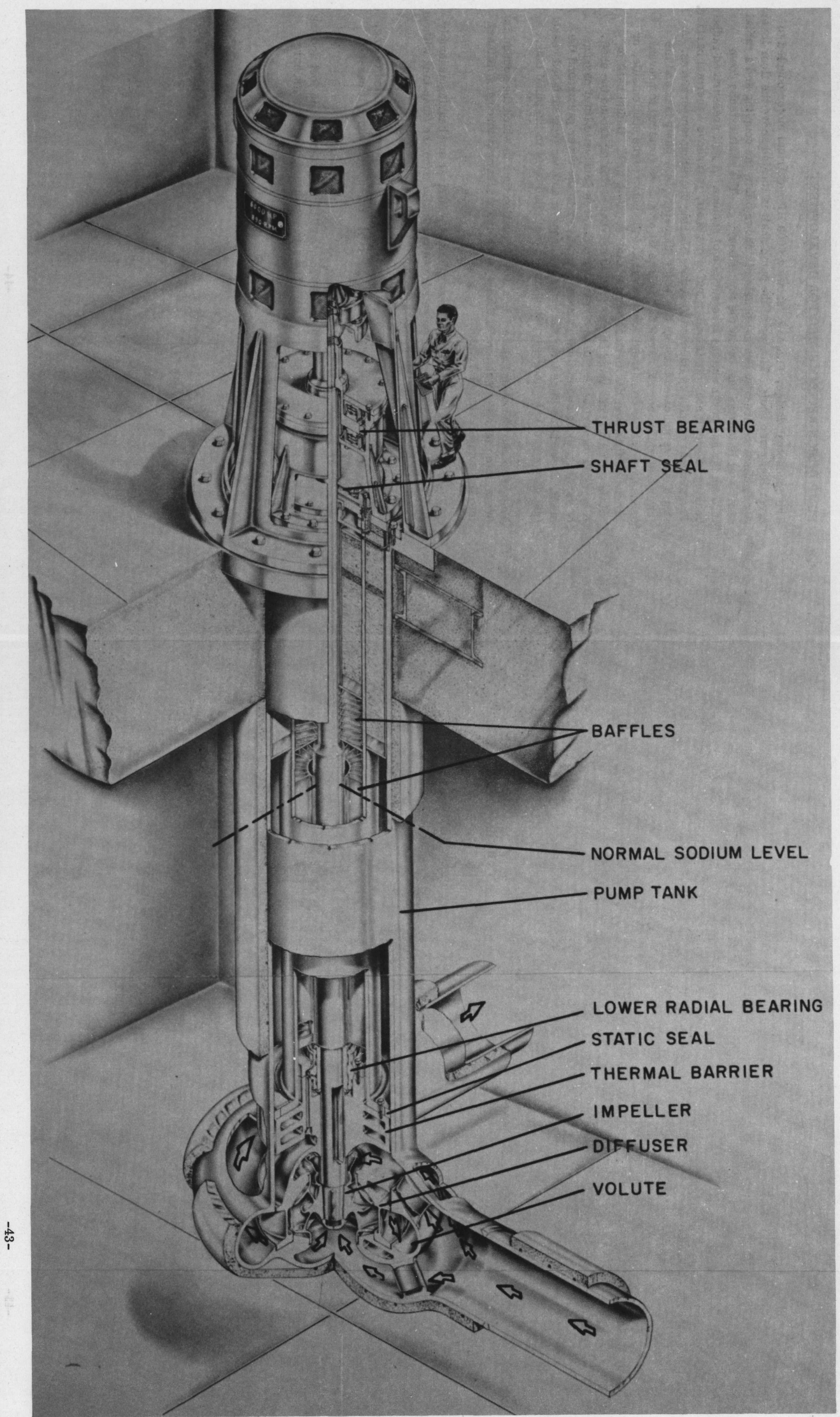

CONCEPTUAL DRAWING

OF A 6000 HP FREE SURFACE

SHAFT SEALED SODIUM PUMP

Figure 7 


\section{0 PUMP DESIGN RECOMMENDED FOR DEVELOPMENT}

The conceptual design, Figure 7, shows a 6000 HP electrical motor coupled to a vertical pump shaft. The top of the pump shaft is located above the floor level. A combination thrust and radial bearing is shown below the coupling and a radial shaft seal is located just below the thrust bearing. The shaft extends down through the gas cover and the free surface of sodium. A self pressurized radial bearing is shown near the impeller end of the shaft, just above a thermal barrier. The thermal barrier is intended to reduce bearing operating temperatures. $\mathbf{A}$ static seal is indicated between the inside diameter of the pump tank and the lower end of the thermal barrier. A close fit or seal is required to prevent excessive leakage from the discharge size of the pump to the suction side, or excessive flow past the thermal barrier into the pump tank. Radial baffling within the pump tank would be required to prevent vortexing around the shaft. A layrinth or vertical baffle is shown above the free surface to limit passage of sodium vapors and to limit convective transfer in the cover gas around the shaft. Equalizing lines from the reactor vessel or another free surfaced vessel (not shown) would be necessary to maintain the liquid level in the pump tank.

The pump end shown in Figure 7 is a double suction type in which the sodium is drawn into the top and bottom of the impeller and discharged through a diffuser into the pump casing or volate.

The pump internals (impeller, diffuser, thermal barrier and bearing assembly, and baffling), as shown, can be withdrawn vertically from the pump tank without requiring pereonnel to work below floor level.

Thermal insulation and heaters are indicated around the pump tank and casing, and radiation shielding is shown at the top of the pump tank where it passes through the floor.

The overall length of this $6000 \mathrm{HP} 890 \mathrm{rpm}$ unit is approximately 45 feet and the diameter of the pump tank is 5 feet. The shown suction piping and discharge nozzle inside diameter are 41 and 36 inches respectively. 


\subsection{PUMP DEVELOPMENT PROGRAM}

\subsection{SCOPE}

It has been shown that liquid sodium pumps will be required in future plants which are many times larger than any sodium pumps which have been built to date. To some extent, these large pumps can be scaled up from present technology, but there are certain critical areas of pump technology which cannot be scaled up dependably, particularily where the large pump has specific problems and limitations not comparable to the 10,000 GPM pumps of today's technology. Three such critical areas are the shaft seals, bearings and hydraulic performance. The nature of these three critical problems and the details of the development required to resolve them are discussed in Section 8.0 of this report.

Various types of sodium pumps have been discussed, and selections have been made from a comparison of the predicted performance characteristics. This development program specifically recommends that the development of sodium pumps proceed along three relatively independent courses:

1. The mechanical centrifugal shaft sealed pump

2. The totally enclosed canned motor pump

3. An advanced sodium pump

The initial effort would begin with parallel development projects on the first two pumps followed, after 18 to 20 months, with the initiation of the advanced sodium pump program.

The advanced pump design is not specified at this time but is left open to include new ideas and possible new developments occurring during the initial stages of the first two pump development efforts.

The program, in an overall sense, provides for the design, development and operational testing and subsequent modifications of at least three types of sodium pumps. Operational testing and evaluation is conducted in a Sodium Pump Test Facility described briefly in Sections 10 and 11 of this report and in detail in the final report on Phase $I I$ for this contract. The testing program will provide comparative operational experience on steady state, and transient performance and general reliability characteristics.

\subsection{DEVELOPMENT PROGRAM AND SCHEDULE}

The sodium pump development program is divided into a logical four phase program and integrated with the projected requirements of large sodium cooled raactor projects as shown in Figure 8. 


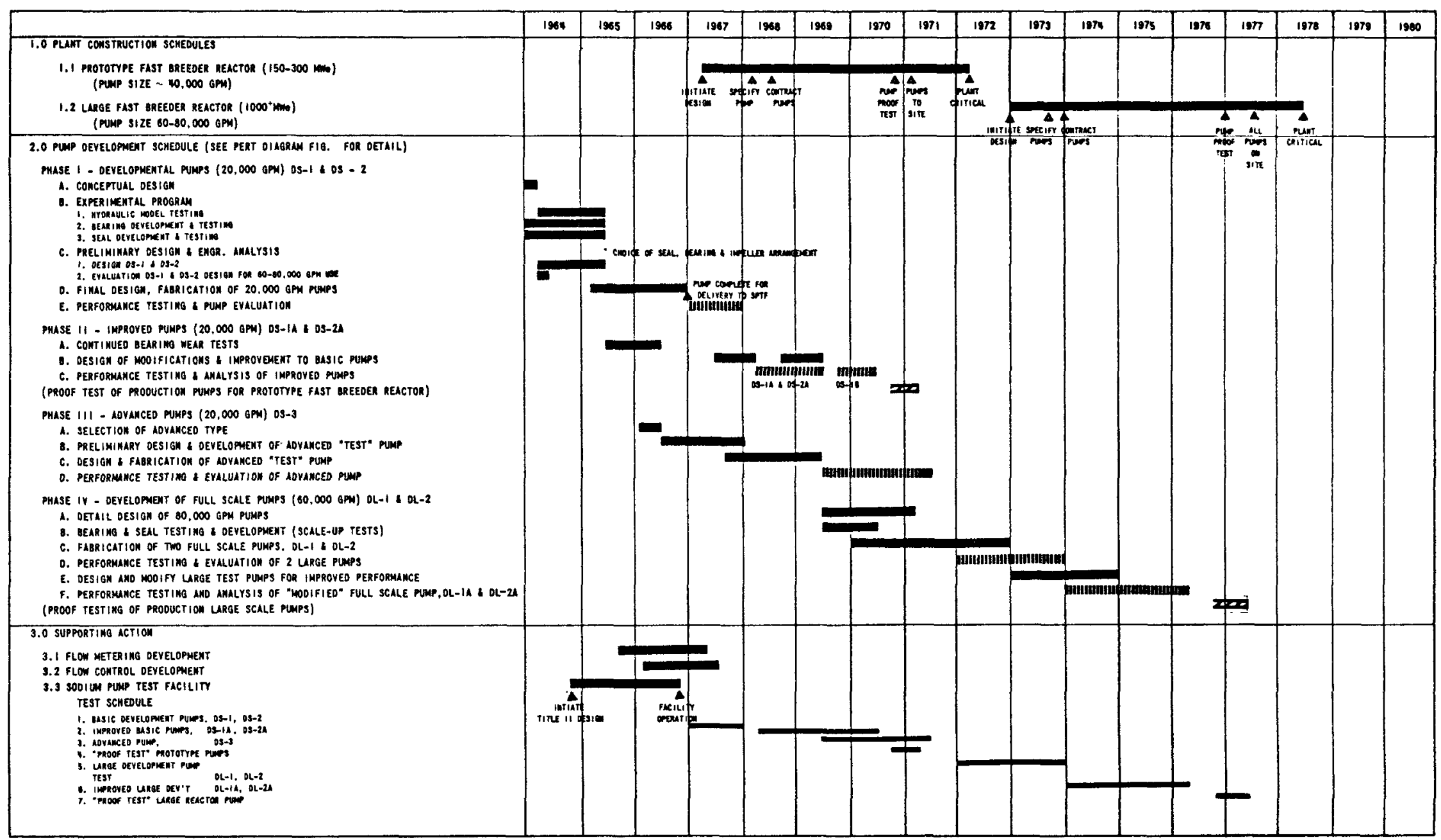

FIG. 8 OVERALL SODIUM PUMP DEVELOPMENT PROGRAM 


\subsubsection{Plant Construction Schedule}

The schedule of pump requirements is based on a consideration of a 200-300 MWe Prototype Fast Breeder Reactor for operation in 1972 and a 1000 MWe Plant by 1978. Significant calendar year dates projected for these plants are as follows:

\section{$\underline{200-300 ~ M W e ~ P r o t o t y p e ~ P l a n t ~} \quad 1000$ MWe Plant}

Initiate Design

Specify Sodium Pumps

Contract for Pumps

Pumps for Proof Test in SPTF

Pumps Delivered to Site

Plant Operational

$$
\begin{aligned}
& \text { lst Quarter - } 1967 \\
& \text { 1st Quarter - } 1968 \\
& \text { 2nd Quarter - } 1968 \\
& \text { 4th Quarter - } 1970 \\
& \text { 2nd Quarter - } 1971 \\
& \text { 2nd Quarter - } 1972
\end{aligned}
$$

$$
\begin{aligned}
& \text { 1st Quarter - } 1973 \\
& \text { 3rd Quarter - } 1973 \\
& \text { 1st Quarter - } 1974 \\
& \text { 4th Quarter - } 1976 \\
& \text { 2nd Quarter - } 1977 \\
& \text { 2nd Quarter - } 1978
\end{aligned}
$$

\subsubsection{Development Program Schedule}

In order to supply important information to the plant designers, it is mandatory to have a history of operating and performance analysis of development pumps prior to the critical dates for specifying sodium pumps. Two years of operation would be highly desirable but may not be possible on the already apparent tight schedule.

The schedule for this four phase program is shown in Figure 8 and the costs are presented in Table 8.

Time dependency PERT networks for the Four Phase Program and the Phase I Program are given in Figure 9 and 10 respectively.

Phase I Development Pumps

In this phase, two parallel contracts for the design, development and fabrication of 20,000 GPM pumps, DS-1 and DS-2, would be initiated. The programs would consist of the following tasks:

A. Conceptual Design of 20,000 and 80,000 GPM pumps (mechanicalcentrifugal pump with shaft seal and totally enclosed motor types). This task would provide the important technical parameters for a full scale sodium pump which would provide the basis for the design criteria for the 20,000 GPM development pumps, DS-1 and DS-2. These parameters would, in turn, supply the technical requirements to the experimental program which follows. 
COST ESTIMATE

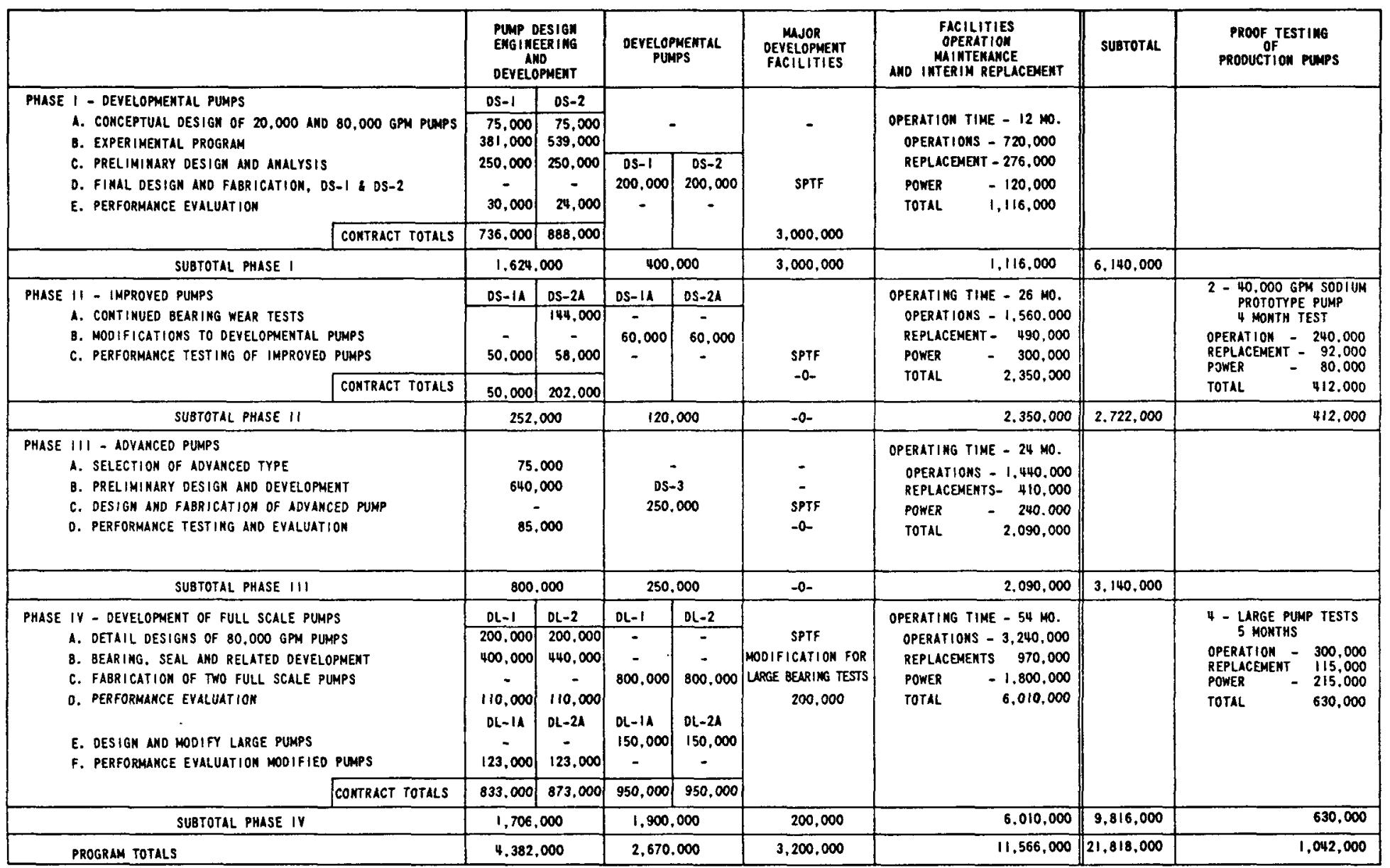

TABLE 8 


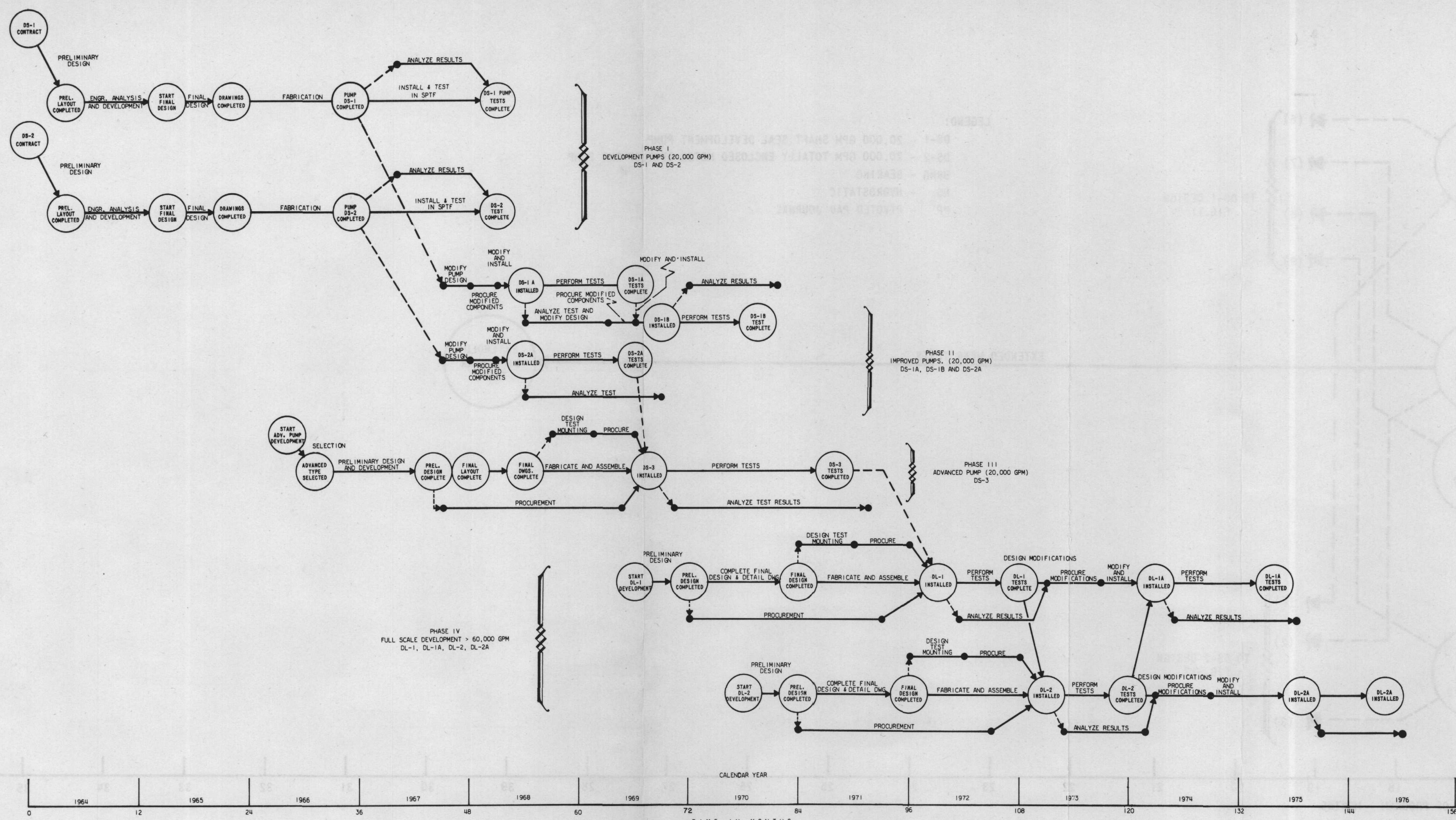


(a)

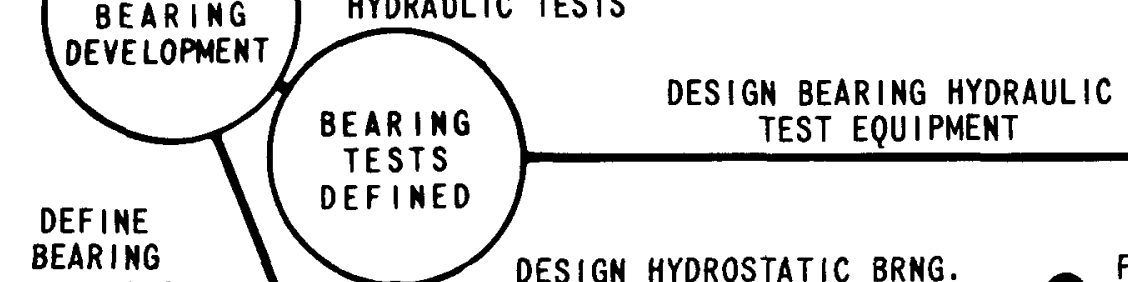

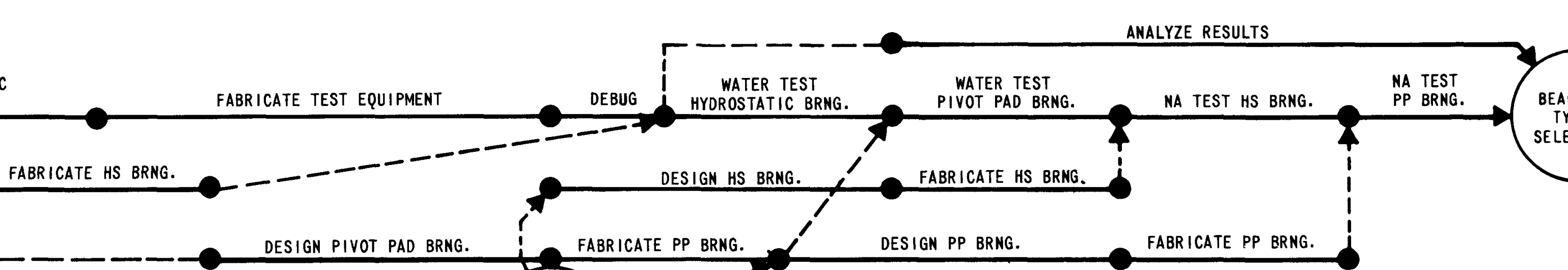

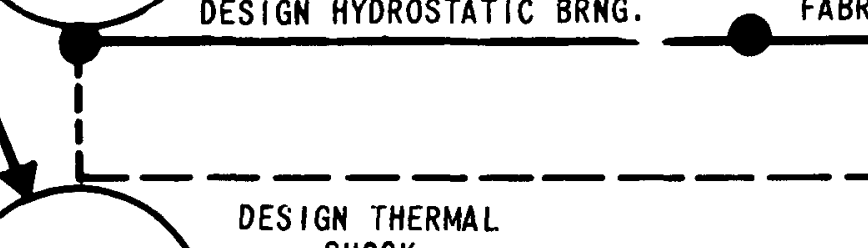

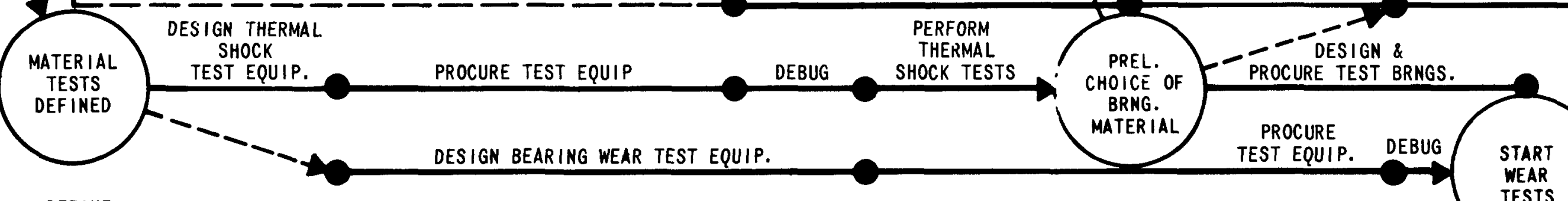

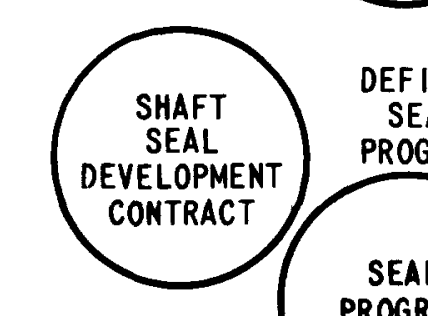

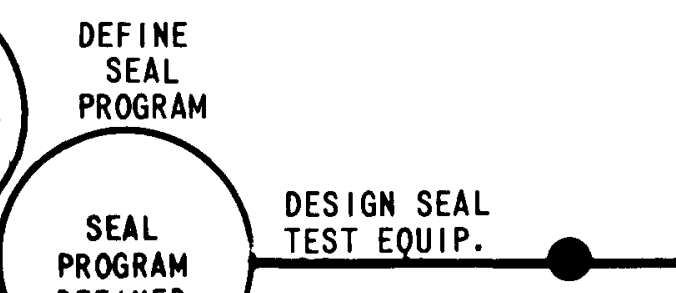

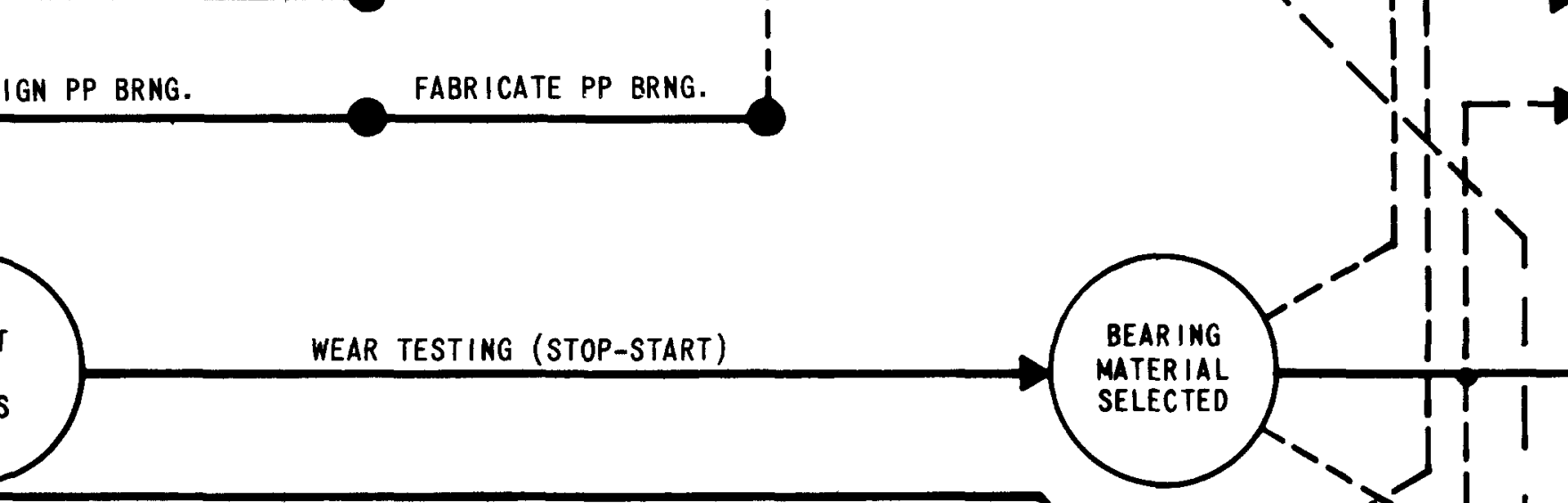

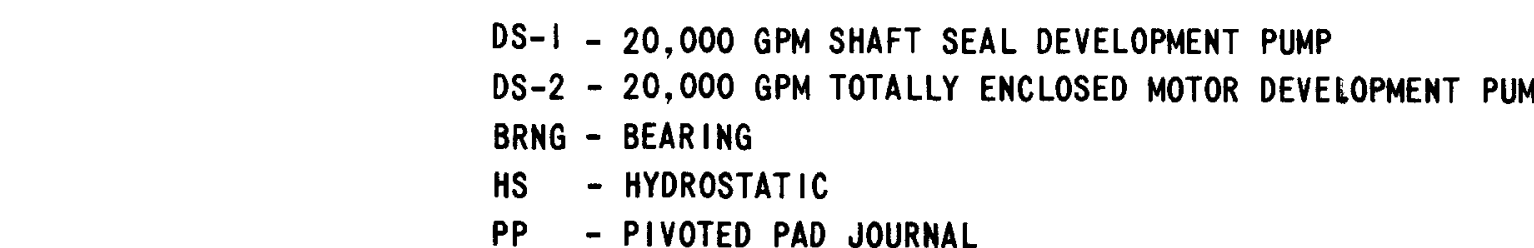

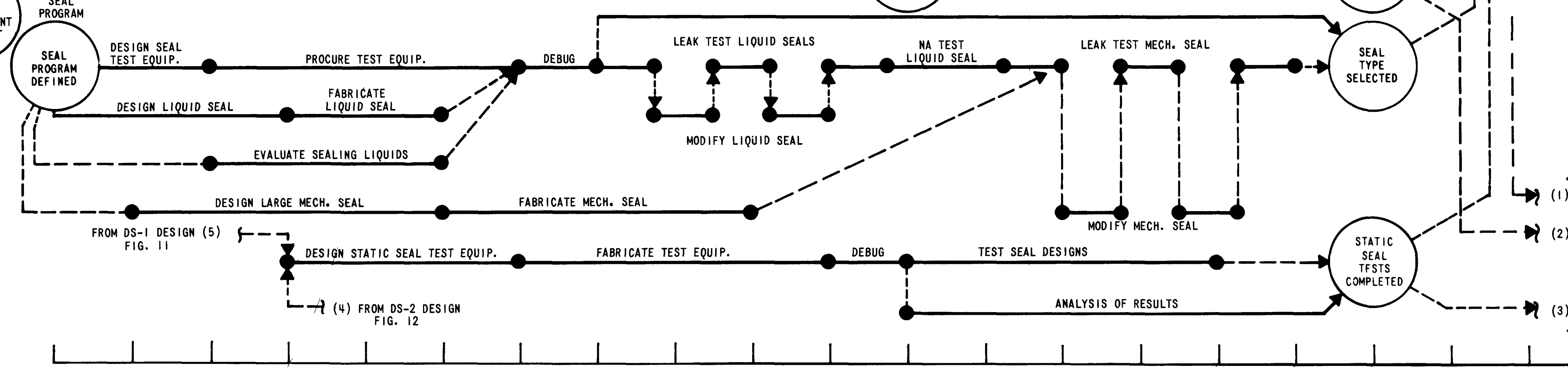

$=$ 


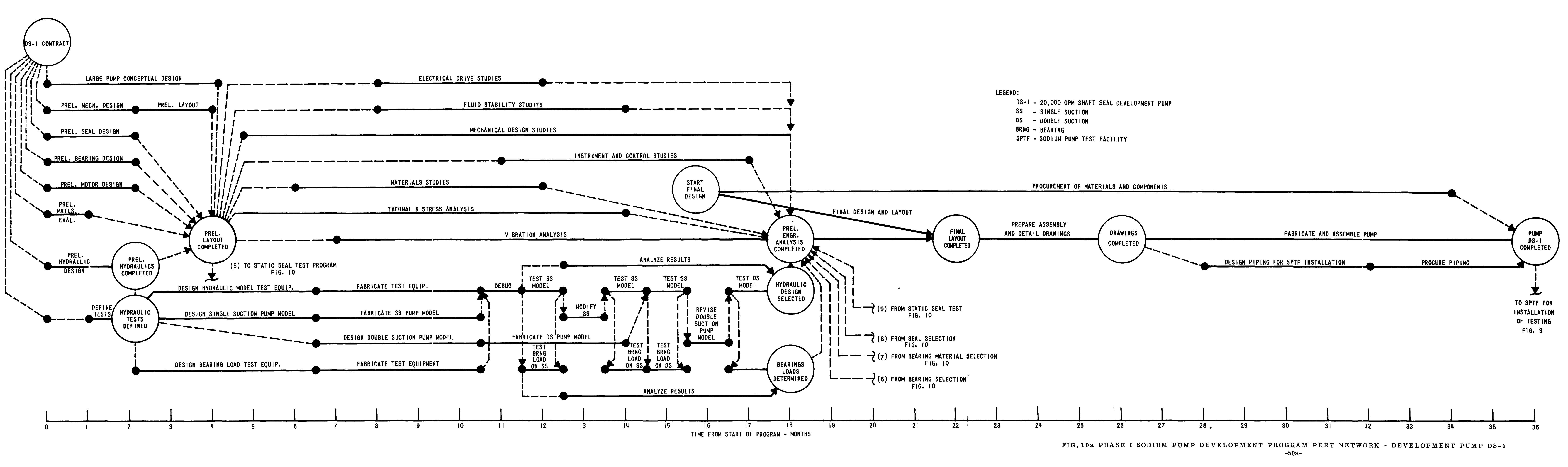




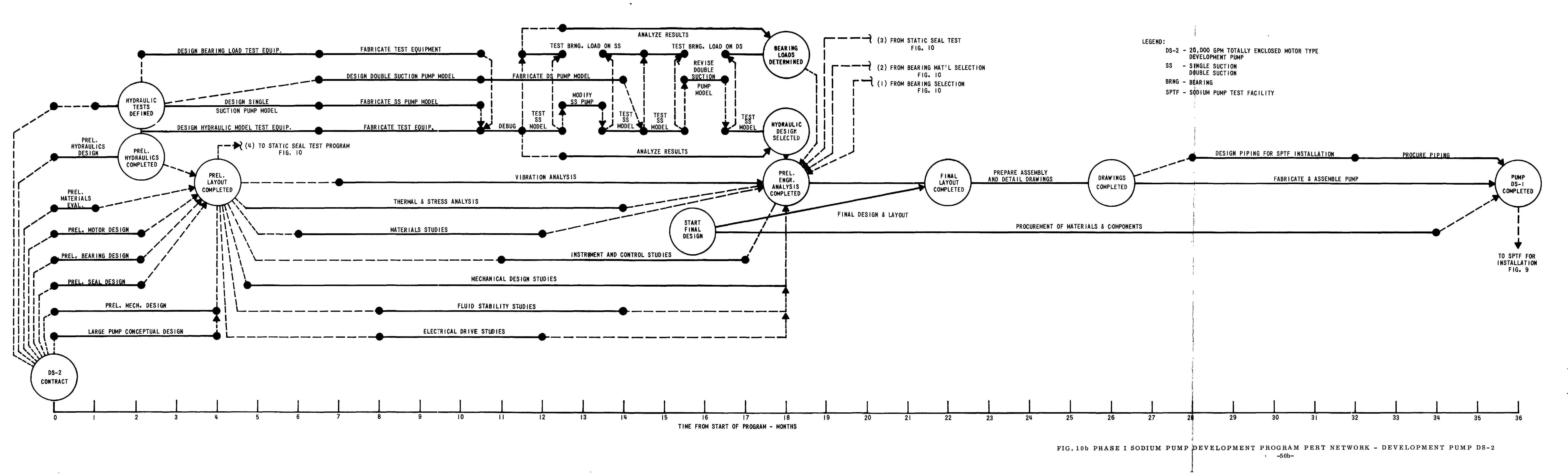




\subsubsection{Development Program Schedule (Cont.)}

B. Experimental Program - including model hydraulic and bearing load tests and full size seal and bearing tests. It is recommended that the bearing and seal test be conducted in a common facility but the other experimental programs would be most advantageously conducted on models more specific to the individual designers, thus requiring parallel facilities and effort. The details of these programs are discussed in Section 9.0.

C. Preliminary Design and Engineering Analysis - the design of the development pumps, DS-1 and DS-2, are undertaken on the schedule indicated and are consistent in concept to the 80,000 GPM design developed in $\mathrm{A}$.

D. Final Design and Fabrication of 20,000 GPM Pumps - from the experimental and analytical studies made in the first 18 months, the final design and fabricating of DS-1 and DS-2 is undertaken. The design and production time required for these two pumps is about 21 months.

E. Performance Testing and Pump Evaluation - the two developmental pumps are then subjected to extensive testing in the Sodium Pump Test Facility and the results evaluated.

\section{Phase II Improved Pumps}

In this phase of the program, some additional bearing wear tests are conducted in the model testing facilities, and the first two development pumps, DS-1 and DS-2, are modified according to the results obtained in Phase I and submitted for new tests. These tasks are included:
A. Continued Bearing Wear Tests
B. Design and Modification of Development Pumps, DS-1A, DS-2A
C. Performance Testing and Analysis of Improved Pumps

No additional facilities are needed in this phase.

Phase III Advanced Pumps, DS-3

Approximately two years after the initiation of Phase I, an advanced pump development program should be considered. At the present time the pumps recommended in Phase I represent the most promising route to the program objectives. However, the possibility of advancing technology producing a more favorable advanced concept must not be overlooked. The Phase III program provides for the further development and testing of such a concept. While the advanced concept cannot be specified at this time, it would appear that continuing improvement of either the more favorable electromagnetic or mechanicalelectromagnetic concepts could lead to the desireability of further development of one of these concepts in Phase III. This phase includes the following tasks: 


\subsubsection{Development Program Schedule (Cont.)}

A. Selection of Advanced Type - from the operating experience of the Enrico Fermi Fast Breeder, the Experimental Breeder-2 and the Hallam Nuclear Power Facility and the experimental development conducted in Phase I, pump technology would have sufficiently progressed to allow the selection, on an evaluated basis, of an advanced sodium pump concept, DS-3.

B. Preliminary Design and Development of the Advanced Pump - following the selection of the advanced pump concept, a combined analytical and experimental program should be undertaken on the critical areas of technology similar to the program conducted in Phase I.

C. Design and Fabrication of Advanced Test Pump - the estimated time for the design and fabrication of this advanced pump is approximately 20 months.

D. Performance Testing and Evaluation of Advanced Pump - the results of the performance tests of the advanced pump would be thoroughly analyzed and compared in detail with the results obtained from DS-1, $2,1 \mathrm{~A}$ and $2 \mathrm{~A}$. At this time, it would be possible to select a preferred and an alternate pump concept for development and demonstration in the full scale sizes.

Phase IV-Development of Full Scale Pumps (60-80,000 GPM) DL-1, DL-2

At this point in the program, there would be a significant amount of pump development information from hydraulic and seal and bearing model tests and from the performance analysis of the basic and improved development pumps. A preferred and alternate pump design for 80,000 GPM application could be selected from this information. It is important that the full scale development and testing begin at this time in order to provide at least one year of operating and performance analysis before a design selection for a pump for the $1000 \mathrm{MWe}$ reactor plant must be made (Third Quarter 1973).

A. Detail Design of 80,000 GPM Pumps - in the Phase I program, conceptual designs of two 80,000 GPM pumps were conducted, these providing a development guide to the early part of the pump program. In this task the detailed design of two large pumps would be undertaken on a slightly staggered schedule.

B. Bearing and Seal Testing and Development - the experimental program would include full size seal tests and near full size bearing and hydraulic tests. New, larger facilities would be required for these tests and could be readily added to the Sodium Pump Test Facility as a service facility, thereby eliminating the need for duplicating large, costly facilities. 
7.2.2 Development Program Schedule (Cont.)

C. Fabrication of Two Full Scale Pumps, DL-1 and DL-2 - the estimated fabrication time for the large pump is 32 months.

D. Performance Testing and Evaluation of Two Large Pumps - testing of the first large pump should begin late in 1971 in order to provide data for the large plant designers in the third quarter of 1973. These pumps would receive extensive testing as noted in Section 11.

E. Design and Modify Large Test Pumps - this rigorous test program would doubtlessly reveal design flaws, weaknesses in performance and factors contributing to reduced reliability. A thorough evaluation of the operating performance of these pumps will clearly indicate areas for improvement. The existing pumps will be modified in conformance with the potential areas for improvement.

F. Performance Testing and Analysis of Modified Full Scale Pumps, DL-1A and DL-2A. - extensive testing of the design modifications would then be undertaken.

\section{3 SUPPORTING ACTION}

The program outline in 7.2 is basically for the large sodium pumps. Additional work must be conducted in other areas of sodium technology relating to high sodium flow rates.

A. Flow Measurement Development - although sodium flow can be measured reasonably accurately with some difficulty in smaller pipe size and flow rate, the accurate measuring of sodium flow at high rates in large size pipes represents a formidable problem. Development should be conducted in parallel with the Phase I Pump Development Program on improved techniques for accuracy and reliability in sodium flow control. These techniques can be proof tested in the SPTF.

B. Flow Control Development - related development on optimum methods and practice of flow control in large systems is needed. The pump tests will require suitable means for varying flow and pressure drop in a convenient and reliable manner.

C. Sodium Pump Test Facility - this facility is the cornerstone for any significant pump development and test program. Its general description and capabilities are given in Sections 10 and 11 of this report and the estimated cost is noted in Table 8. A more detailed design and cost estimate will be issued shortly as the Phase II report of this contract. 


\section{Sodium Pump Test Facility (Cont.)}

The construction schedule of this facility is estimated to be 24 months and constitute a critical schedule item on pump development as noted by the pump test schedule in Figure 8. From the 4th Quarter of 1966 there seems to be a continuing requirement for testing of one or more pumps of sizes from 20,000 GPM to 80,000 GPM. This facility would also be used to proof test production pump models as shown by the solid black line in Figure 8.

The facility will also have considerable value in testing many flow related problems in sodium systems and cores:

1. Control and stop valve testing.

2. Flow induced vibrations in reactor core models.

3. Full scale fluid dynamic tests in steam generators and intermediate heat exchangers.

4. Flow measurement.

5. Isothermal materials testing for corrosion, erosion etc.

6. Sodium purification system.

\subsection{SODIUM PUMP DEVELOPMENT COSTS}

The cost estimates for the four phase program are presented in Table 8 where it can be noted that the cost for each phase is segregated into four main categories:

1. Engineering and Development

2. Cost of Developmental Pumps

3. Major Facilities Costs

4. Facilities Operation and Maintenance

The overall program requires approximately $\$ 4,400,000$ for the development of liquid metal pump technology. This includes all conceptual design effort, critical problem solving through experimental model, preliminary design and performance evaluation.

Five developmental pumps ranging in size from 20,000 GPM to 80,000 GPM are required which will test competing total pump assemblies. The estimate of 2,600,000 includes the cost of all developmental pumps and their modifications and improvements. 


\subsection{SODIUM PUMP DEVELOPMENT COSTS (CONT.)}

The cost of a Sodium Pump Test Facility has been estimated in only a highly preliminary way to be $\$ 3,000,000$ with projected modifications for large bearing tests costing an additional $\$ 200,000$. The SPTF is vital to the development and testing of large, reliable sodium pumps and is required early in the program (by 1966) but the large bearing test modification can be added in 1969 .

The cost of operation, maintenance and interim replacement and electrical power consumption is the largest single cost component of the entire program at $\$ 11,566,000$. This estimate is based on almost full time operation of the development pumps. If endurance testing were eliminated, this cost could conceivably drop to only $\$ 3-4,000,000$ but its inclusion is recommended, at least at this phase of the program planning.

The separation of the Pump Development Program into four phases is a logical orientation of development effort and progress. The effort from Phase $I$ is completed and Phase II and III well along before final specifications have to be established for the Prototype Plant Pump. The development effort in Phase I is rather high in relationship to the pump costs because it is imperative that the early phases of this program give careful consideration to the optimum solution to the sodium pump problems.

Upon detailed inspection of the schedule in Figures 8,9 and 10 and the cost in Table 8 , it is apparent that this program could be conveniently implemented through several related but essentially independent development programs:

Scope

Approximate Value

1. Design, development, fabrication and performance analysis of a 20,000 GPM Mechanical shaft sealed pump, DS-1

$\$ 936,000$

2. Design, development, fabrication and performance analysis of a 20,000 GPM totally enclosed pump, DS-2

3. Incremental extension of (1) and (2) modification for improvements and subsequent testing

4. Design, development, fabrication and performance analysis of a 20,000 GPM liquid sodium pump of advanced design, DS-3

5. Design, development, fabrication and performance analysis of an 80,000 GPM pump of a preferred design

$1,783,000$ 


\subsection{SODIUM PUMP DEVELOPMENT COSTS (CONT .)}

$\underline{\text { Scope }}$

Approximate

Value

6. Design, development, fabrication and performance analysis of an 80,000 GPM pump of an alternate design

$\$ 1,823,000$

7. Design and construction of a sodium pump test facility

$3,000,000$ plus 200,000 modification

8. Operations, maintenance, and power supply for the SPTF

$11,566,000$

The total program cost of $\$ 22,000,000$ does not include the cost of proof testing of production pumps. This total cost seems entirely commensurate with the objective of developing large sodium pumps having excellent performance characteristics and proven reliability. 


\subsection{DEVELOPMENT PROGRAM FOR MAJOR PROBLEM AREAS}

\subsection{SCOPE}

The design of reliable large sodium pumps of the types selected for development, i. e. mechanical pumps with shaft seals and mechanical pumps with totally enclosed motors, require the solution to three major problems, namely

1. Development of seals for large diameter shafts

2. Development of large diameter sodium lubricated bearings

3. Development of high capacity pumps with low net positive suction head requirements.

The problems of bearing and pump hydraulic design are of equal importance for both of the pump concepts under consideration. The problem of seal design is primarily of importance to the shaft sealed design as the sealing requirements of a totally enclosed motor considerably less stringent.

The experimental program is to investigate those basic concepts which appear feasible for use in future large designs and to determine their basic characteristics and fundamental design information necessary to permit design and incorporation of such components in development pumps. The developmental pumps will be subsequently extensively tested in loops under simulated operating conditions.

\subsection{SHAFT SEAL DEVELOPMENT}

\subsubsection{Discussion of Problems}

The criteria which follow represents the factors which are relevant to shaft seal selection and development for large, free surface sodium pumps.

A. Proven reliability - i. e, the seal should have a good record under actual services. Some of the qualities which are embodied in the term "reliability" are:

(a) Conservative design - no areas of marginal operation.

(b) Ruggedness - ability to stand abuse during operation and installation.

(c) Simplicity - low chance of misuse.

(d) Low susceptibility to dirt, foreign matter and the usual hazards of operation.

(e) Minimum damage in the event of malfunction. 


\subsubsection{Discussion of Problems (Cont.)}

B. No contamination of liquid sodium - A seal fluid, if required, should be either compatible with molten sodium, or capable of being prevented from coming in contact with it.

C. Low leakage - Zero cover gas leakage is ideal. If this is not possible, then leakage rates must be kept low to minimize cost. Possibility of the release of contaminated products should also be considered.

D. Long life - indefinite life is ideal.

E. Easy maintenance - the replacement and/or inspection of the seal should be mechanically simple to perform. Day-to-day servicing should be a minimum. The presence of the seal should not complicate service and maintenance to other parts of the machine.

F. Low power consumption - The seal should not lower appreciably the efficiency of the machine, or be the source of undue heating and allied effects.

G. Ease of manufacture - Tolerances should not be unduly restrictive. Parts should be capable of manufacture by conventional equipment. Materials should be readily available with regard to composition, properties, size, etc.

H. Low cost.

After reviewing the many types of seal configurations, those seals which appear promising in meeting the design objectives for this application may be categoruzed as (a) rubbing seals, (b) liquid seals, (c) gas seals, and (d) mercury seals.

\section{A. Rubbing Seals}

Figure lla illust rates schematically the rubbing seal, or mechanical contact seal, as it is often called. Although "circumferential" sealing could be affected by rubbing on the journal surface, low leakage rates dictate "axial." sealing against a runner as shown in Figure lla. As the diameter of the shaft would be about 12 inches, the sealing interface of the runner would necessarily be of the order of 18 inches in diameter. At $900 \mathrm{rpm}$, this would entail a rubbing speed of about 4200 feet per minute. Under these conditions, lubrication with oil would be necessary to prevent rapid wear and excessive heat generation. Many types of these seals using various mating materials are commercially available, and although a seal of this type of the required size is hardly an "off-the-shelf" item, it can be considered within the state. of -the art. Moreover, it should be mentioned that operation speeds above $4200 \mathrm{fpm}$ will take it out of this category and require extensive development work. 


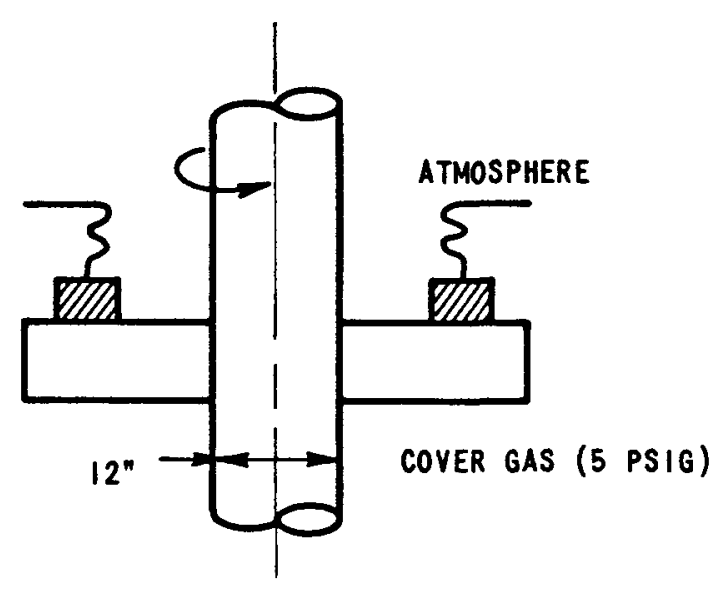

a. MECHANICAL RUBBING SEAL

1
0
0

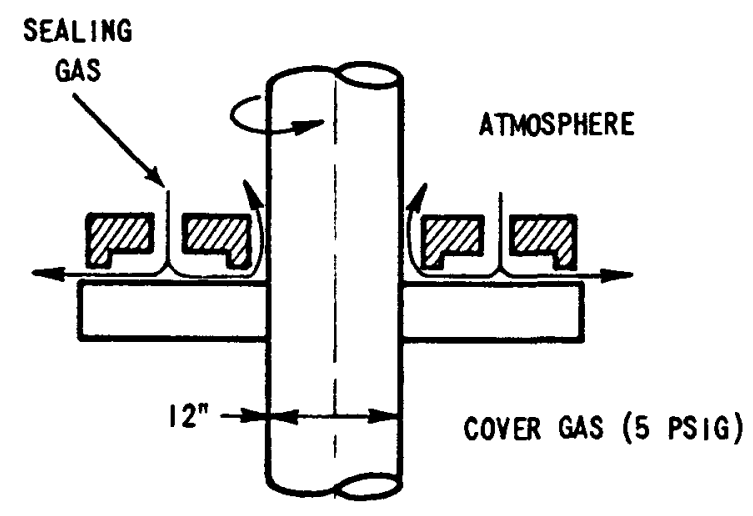

c. GAS SEAL

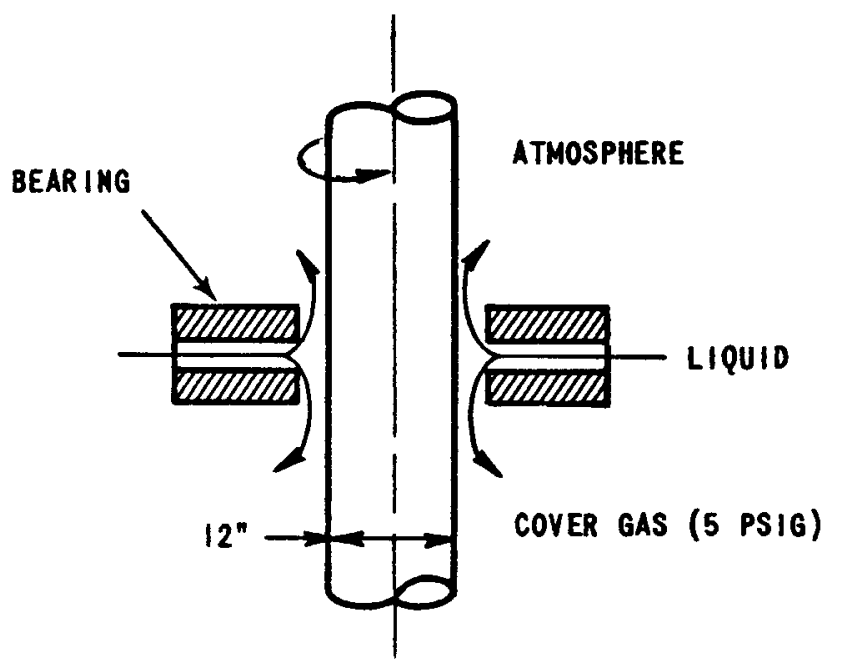

b. LIQUID SEAL

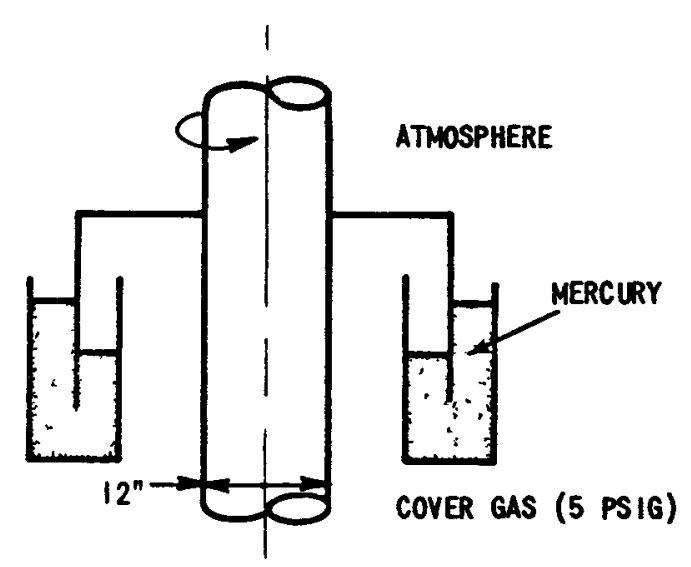

d. MERCURY SEAL 


\section{A. Rubbing Seals (Cont.)}

Hence the design experience gained with this seal, should it be employed, will be of little value in designing larger pumps with the same or higher operating speeds. The designer would again be confronted with a sealing problem which has to be solved anew.

This type of seal has the following advantages: it is simple, cheap, readily available and would require a relatively short development time to adapt it to this application. In smaller size it has shown reasonably good reliability. Cover gas leakage at 5 psia would be insignificant and because of the small amount of oil required for lubrication, oil leakage into the system could be easily prevented by proper design.

In addition to the limitations pointed out above, rubbing seals would also have the following disadvantages:

(a) Limited life - which could be very short.

(b) Must be made and assembled with a high degree of precision. The mating surfaces must be lapped flat within a few light bands and the precision must be maintained in the equipment.

(c) The seal cannot be split readily. This complicates installation and replacement and tends to compromise the design of the machine itself.

(d) The seal has a PV (contact pressure $x$ rubbing speed) limit. Larger sealing diameters or higher speeds may render this type of seal impractical.

(e) Sealing against higher cover gas pressures, if it should be necessary, may not be possible.

\section{B. Liquid Seals}

Figure 1lb shows a schematic diagram of a liquid seal. Liquid is injected under slight pressure so as to form a sealing film between the shaft and the seal ring. In contrast to the rubbing seal, there is no contact between the parts and hence no wear. The seal, therefore, has indefinite life. There is no cover gas leakage past the interface. This type of seal has been used for many years in central station service to contain hydrogen gas in large electric generators where surface speeds and cover gas pressures far exceed those being considered in this study. They have proven to be extremely reliable.

While the design shown in Figure llb seals radially, it could also be made to seal axially against a runner. The radial configuration facilitates a split construction thereby simplifying the design. 


\section{B. Liquid Seals (Cons - )}

This type of seal possesses the following advantages:

(a) Proven reliability, especially in large sizes.

(b) Indefinite life.

(c) The seal is easily constructed and is separable radially. This permits easy replacement if necessary or removal, easy inspection, and simplified the construction of the machine.

(d) The seal does not require high precision in manufacture and installation.

(e) The sealing capability is independent of operating speed, i.e., there is is no PV limitation.

(f) The seal is capable of sealing against a high cover gas pressure ( 30 psig is standard practice on hydrogen cooled generators).

It has the following disadvantages:

(a) Back-up equipment and auxiliaries such as pumps, valves, etc。are required.

(b) Relatively large quantities of liquid (as compared to the mechanical seal) are required. However, contamination of sodium can be prevented by proper design.

(c) Some cover gas replenishment may be necessary because of absorption by the liquid sealant, However, this can be minimized by proper design.

\section{Gas Seals}

Figure 1ln illustrates sohematically one type of gas seal. This seal can be designed to permit the sealing gas (which would be argon in this case) to (a) leak into the system as shown, or (b) to permit the cover gas to leak out of the system. In either case, leakage will occur and must be provided for the machine. The seal is actually a gas bearing. The thickness of the operating film and the pressure within it dictates the amount of leakage which will occur. The operating film can be generated either hydrodynamically or hydrostatically: For this application, hydrostatic techniques would be used. Since the rubbing surfaces would be separated by a gas film, there would be no wear and liffe would be indefinite provided rubbing due to dirt, etc. did not occur.

Although the seal could be made so as to seal radially in a manner similar to 


\section{Gas Seals (Cont.)}

the liquid seal, the very high leakage rates which would thus be encountered would be impractical because of the higher radial clearance which would be required (say .005 -.006 in. on a diameter of 12 inches) to prevent seizure. As the leakage varies with the cube of the film thickness (clearance) this would result in intolerably large flow rates. Much smaller film thicknesses can be maintained with comparative safety when sealing axially against a runner as shown. However, in order to keep leakage rates low, the film thicknesses must be kept very small, in the range of 0.005 to 0.001 inch. While this can be done, from a practical viewpoint it is no small feat when one considers the size of the bearing and the size of the attendant machine components. Scrupulous attention to all design details of the machine will be necessary to avoid mechanical and thermal distortions which could nullify completely the small film thickness, thus causing contact, and rapdr failure of the seal.

The most cutstanding advantage of this seal is that it uses gas as a sealant. This completely eliminates any contamination problem. (There could passibly still be some ingress of air into the system through the phenomena of backdiffusion, but this would be so small as to be probably negligible).

The gas seal has the following disadvantages:

(a) Unprover reliability - gas bearing seals of this type have been used successfully on small shafts (up to $2 \mathrm{in}$. diameter) running at very high speeds $(15,0.00 \mathrm{rpm})$. They must be regarded, however, as extremely delicate high precision components requiring scrupulous care in manufacture, installation, and operation. The large size seal requized for this application may be incompatible mechanically with the small tolerances and operating film thickness required, Small orifices which moy become clogged are required on the seal design. As no lubricant is present, the seal would have relatively low tolerance to momentary rubs that may occur.

(b) Leakage - the system must be designed to allow for a controlled amount of leakage. This is not a zero leakge seal.

(c) Back up equipment such as valves, sesilant source (compressor). etc. is required.

(d) The seal cannot be split without some loss in precision of the mating surfaces thereby complicating the inspection, replacement, or removal of the seal and also the design of the machine in general.

D. Mercury Seals

Figure lld indicates schematically one type of mercury seal. Mercury seals 


\section{Mercury Sea] (Conit)}

can be both static and dynamic. The design shown does not effectively use the dynamic pressure which can be generated by centrifugal force to enhance sealing, but this can be done by proper design. Dymamic sealing againat very high cover gas pressures can be accomplished utilizing this effect.

Many patems have been issued on this type of seal. It has been successfully on small equipment, particularly to seal against sacuum. However, its application in the size being considered here is unknown.

The mercury seal possesses the following advantages: It is very simple, requiring no fine clearances, and hence would be easy to make, install and service. It is a truly zero leakage seal, and as there are no rubbing parts would also have indefinite life. It also has the capability of sealing against high cover gas pressure. It is conoeivable that little back-up equipment would be necereary.

However, the mercury seal has unproven reliability in the size being considered for this application. Extensive development may be required. The choice of materials which will allow $\mathrm{Hg}$ to wet the surfaces and yet withstand its corrosive effects may be difficult. The fact that $\mathrm{Hg}$ vapor is toxic does not appear to be a serious problem, but should be taken in account.

One of the most serious disadvantages is that $\mathrm{Hg}$ reacts with molten sodium to form amalgams and compounds which could be harmful in the reactor.

\subsubsection{Experimental Promam}

Objective - The objective of this program is to determLne experimentally the performance cha racteriatice and fundamental information necessary to design a shaft soal suitable tos on large sodium pumps.

In view cf the demonstrated successful application of liquid type seals for use on large shafts (sealing large turbo-generatora) and on the general advantages of the liquid seil previously cited, the primary emphasis of the program is directed to the investigation of liauid type seals. In the event that unforseen difficulties render the linguid seal inadequate, the rubbing seal would be a logical back-up choice. Rubbing seals probably provided the quickest method of getting a pump into operution, but the poor reliability and higher maintenance requirements make such seals less desirable. A parallel investigation of larte rubbing seals will be performed as a backup to the liquid seal design.

Testing - The testing program will involve operating both fluid and mechanical seals under simulated operating conditions in order to determine:

1. Materials performance 


\section{Testing (Cont.)}

2. Leakage rates (effects of temperature and pressure)

3. Fluid suitability and safety of operation

4. Effects and type of failures

5. Wear and expected life data

The liquid seal program will include the selection of potentially suitable seal fluids and their evaluation on the basis of test performance and compatibility with sodium. Experimental models of liquid seals will be designed, fabricated and tested for general performance and leakage under varying gas pressures and subsequently under environmental conditions over a free sodium surface. The program will be carried out with a shaft diameter of approximately 6 inches which it is believed will provide adequate design information to permit engineering scale up of a successful liquid seal to full future pump size with a minimum of additional testing.

The program will also include the fabrication and testing of a number of mechanical seal configurations and materials for use on nominal 6 inch shafts. Wear rates and leakage will be determined. If liquid seals prove infeasible, a continuing program of test and development is anticipated as being required for mechanical seals. This results from the relatively poor sealability of this type of seal and the necessity to essentially test full scale seals.

Test Apparatus - The test apparatus would consist of a shaft (approximately 6 inches in dia.) and shaft seal driven by a variable speed motor. A vessel containing argon blanketed sodium would be used to simulate the free surface in the actual pump tank. Means would be provided to vary gas pressure, temperature and sodium level.

\subsubsection{Schedule and Cost}

A total time of 18 months has been estimated as necessary to complete the Phase I experimental program for seal development. The cost of the program is estimated to be $\$ 150,000$. The schedule and cost are shown on Figure 12 .

On the basis of an overall program keyed to the requirements of a prototype fast breeder reactor scheduled for initial operation in 1972, it is estimated that this seal program should be initiated by January 1964. The relationship of this esperimental program to the overall program is shown on Figures 8,9 \& 10 .

\section{3 BEARINGS DEVELOPMENT}

\subsubsection{Discussion of Problems}




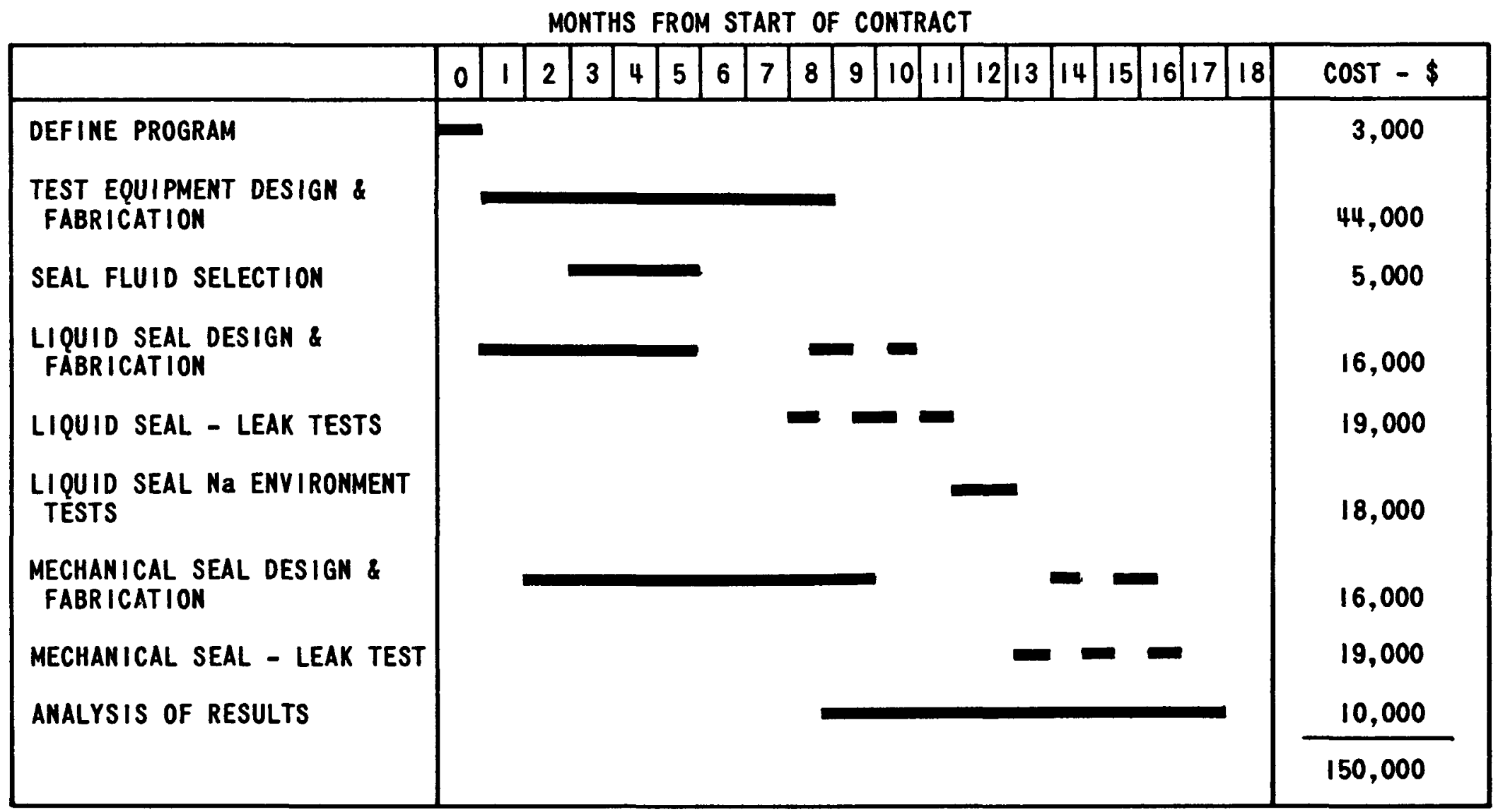

FIG. 12 SEAL EXPERIMENTAL PROGRAM SCHEDULE AND COST 


\subsubsection{Discussion of Problems (Cont.)}

The criteria for the selection of bearings include:

A. Proven reliability - the discussion of this topic under Section 8.2.1 "seals" applies here as woll.

B. Long life - the relative inaccessibility of the beating indieates that $1 \mathrm{mg}$ life is especially desirable. Some of the factors which enter into this aspect are:

1. Ability to withstand frequent start-stops.

2. Ability to operate at reduced speeds when a full fluid film is not present.

3. Ability to withetand thermal shocks, dynamic loads abd vibration.

4. Good material compatibility with molten sodium at $1200^{\circ} \mathrm{F}$.

5. Ability to withstand prolonged operation in reverse direction of rotation.

C. Easy maintenance - the bearing should be capable of being easily inspected and/or replaced. Its presence should not unduly complicate the service on the otner parts of the machine.

D. Low pewer consumption - the bearing should not lower unduly the overall efficiency of the machine, nor be a troublesome source of heat generation.

E. Ease of manufacture - tolerances should not be unduly restrictive. Parts shoild be capable of manufacture by conventional equipment. Materials should be readily available with regard to composition, properties, size, etc.

F. Low costs

It is realized that no bearing will fulfill all the above criteria. Moreover, each of the above yardsticks is subject to personal interpretation as to relative importance and desirability. Nevertheless, all the factors should be considered and evaluated.

Two types of bearings appear promising for this application: (a) the hydrostatio journal hearing, (b) and the pivoted-pad journal bearing (Figure 13). The former utilizes a source of pressure outside the bearing while the latter depends upon the relative motion of the parts to generate pressure with the film. The performance of the pivoted pad bearing, therefore, depends upon the speed of operation. Normally, the hydrostatic bearing is not dependent on speed, but if the external pressure source for the bearing is the pump itself (which is presently being considered because of the great design simplification which results), the hydrostatic bearing also becomes speed dependent. 


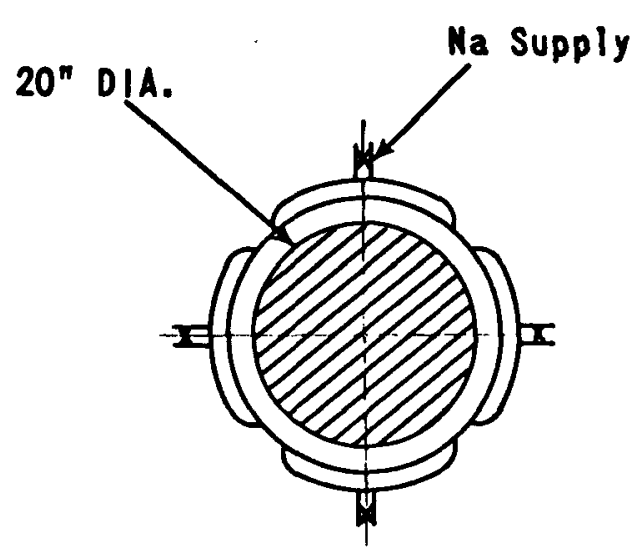

HYDROSTATIC JOURNAL BEARING

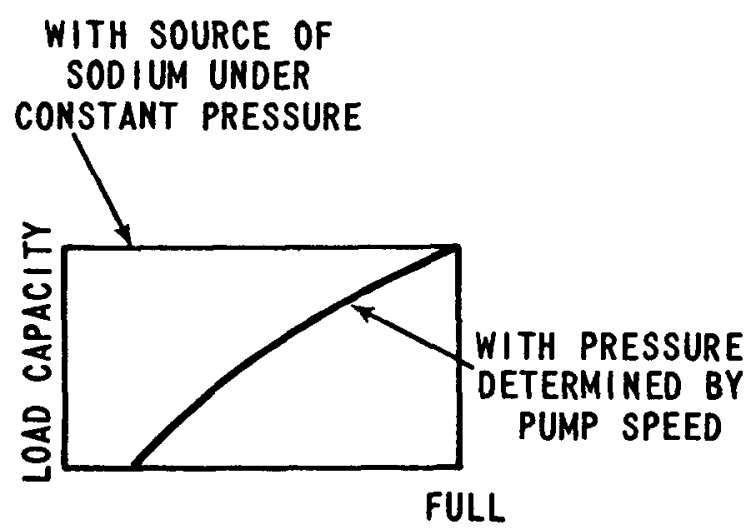

SPEED HYDROSTATIC BRG.

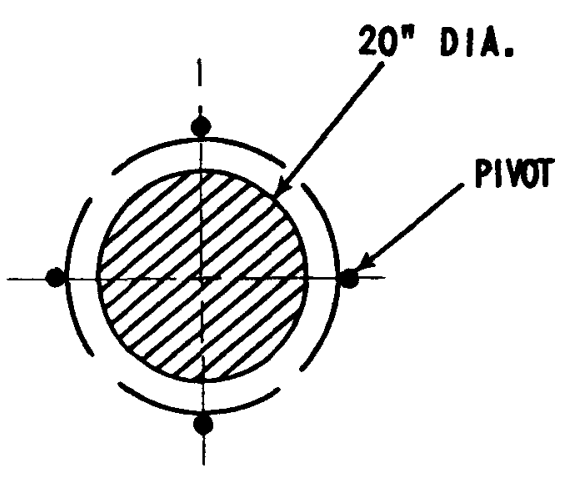

PIVOTED PAD JOURNAL BEARING

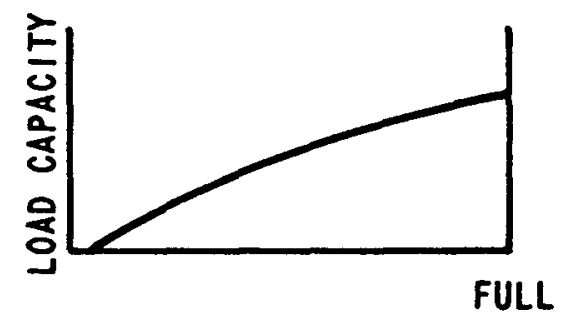

SPEED PIVOTED PAD BRG.

FIG. 13 COMPARISON OF HYDROSTATIC \& PIVOTED-PAD

E. D. SK. 301876-B

JOURNAL BEARINGS 


\subsubsection{Discussion of Problems (Cont.)}

A. Hydrostatic Journal Bearing (Figure 13a)

The main advantage of this bearing for the particular conditions found in this pump is its high load capacity at the maximum speed of $900 \mathrm{rpm}$. The load capacity falls off quickly with speed, but is probably still superior to the pivoted-pad bearing up to the point where initial contact occurs. It operates equally well in either direction of rotation and is probably stable with respect to whipping (however, this should be determined). It is easy to split, thus making for easy assembly and disassembly on the shaft. It has shown reasonable reliability in similar applications in smaller sizes.

On the other hand, the bearing offers little supporting area for operation in the low speed range where rubbing may occur; consequently, the wear rate may be high. It may require as much as $7 \%$ of the total flow capacity of the pump. Moreover, if the design is chosen so as to prevent contact at the lowest operating speed, the size of the bearing could become very large; thereby not only complicating the overall machine design, but also absorbing much of the capacity of the pump when operating at maximum speed. It requires provisions for self-alignment in the housing and orifices for flow regulation which may become plugged. The bearing may be difficult to manufacture in the size required out of materials such as carbides, etc.

It is obvious that some of the above objections can be completely eliminated if a separate pressure source were used and this factor should not be overlooked. The advantages of such a system must be weighed against the complication that results in the overall design of the machine. This decision must await actual design calculations of the bearing sizes, loads, speeds, etc.

B. Pivoted-Pad Journal Bearing (Figure $13 \mathrm{~b}$ )

The pivoted-pad journal bearing has a number of advantages which are of a practical nature. It is inherently self-aligning and consists of a number of elements, loosely held, so that fouling by dirt is unlikely. It may be easier to make, in that the elements, being comparatively small, are readily available materials in the sizes required. It provides considerable support area for operation in the low speed range. Moreover, it is inherently stable with regard to whip and operates equally well in either direction of rotation. Finally, it does not require large additional pump tlow to satisfy its own bearing requirements.

The serious disadvantage of the pivoted-pad bearing is its poor load capacity in relation to the hydrostatic bearing in this relatively low speed application. At maximum speed the bearing could be designed to operate on a film of reasonable thickness, but still small in comparison to a 


\section{B. Pivoted-Pad Journal Bearing (Cont.)}

hydrostatic bearing of the same size. It is felt, therefore, that contact would occur at a considerably higher speed than would be the case with its hydrostatic bearing. However, this objection to the pivoted-pad bearing would not be critical if (a) the contact speed were below $1 / 3$ the maximum speed, or (b) the mating journal bearing materials were highly compatible and gave negligible, or minor wear. These questions can only be resolved by test.

\subsubsection{Bearing Development Program}

Object - The object of this program is to develop a radial bearing capable of meeting the requirements of future large sodium cooled reactor coolant pumps.

Aside from selecting the bearing type and obtaining the analytical tools required for the design of bearings, a program for the measurement of actual pump bearing loads is necessary. While test data will be obtained that will enable a bearing to be designed for any given conditions, some estimate of the actual radial bearing load is required to size a bearing for a given application. Also, the materials and method of fabrication must be evaluated for large diameter bearings.

The program may then be divided into three separate tasks:

1. Selection of bearing type and gathering of design data.

2. Determination of pump bearing loads.

3. Development of fabrication techniques and materials evaluation for large diameter sodium bearings.

Task 1 - Selection and Hydraulic Testing

If a source of sodium under constant pressure were available, a hydrostatic bearing would offer a clear advantage over a pivoted-pad bearing in this application because it can be designed to give a greater load capacity at full speed, see Figure 13. If the hydrostatic bearing is pressurized from the pump, however, the more important consideration may be, which of the two bearings can operate best under the low speed conditions. This must be resolved by a test program.

Testing - Water testing on models of hydrostatic and hydrodynamic (pivoted-pad) bearings to obtain design data so that both types can be evaluated. A comparison of load capacity throughout the entire speed range and general performance during starting and operating conditions will be investigated. This data, and actual pump radial thrust data, will be needed before selection of bearing type or bearing sizing can be made. After water testing either or both types would be 


\subsubsection{Bearing Development Program (Cont.)}

Testing (Cont.)

operated in sodium in order to get load, stability, and film data under aotual conditions.

Test Apparatus - A bearing test stand consisting of a variable speed drive, shaft and bearing, suitable water/sodium container, means of varying fluid pressure and temperature, and instruments to collect bearing test data (pressures, stability, film thickness, etc.) will be required. It is anticipated that 6 inch diameter bearing models will be adequate for these tests.

Back-uy or Alternate - If neither the hydrostatic or the pivoted-pad bearing operates sutisfactorily under the variable speed conditions (low speed ranges), increasing the supply pressure by a supplementary pump can correct the diffioulty،

\section{Task 2 - Bearing Load Determination}

Testing - As mentioned in Task 1 , testing actual pump radial thrust data is necessary in order to select and size the bearings. It is anticipated that this data would be obtained during pump model testing; however, separate journal or bearing mounts and load pickup apparatus will have to be installed in the model test apparatus during load determination testing.

Apparatus - Load determination apparatus to measure shaft load or bearing reaction loads will either be buill into the pump model test stand, or be designed for installation into the test stand during pump model hydraulic testing.

\section{Task 3 - Bearing Materials Selection and Fabrication}

Discussion - Some of the bearing materials known * to be competible with high temperature sodium are listed in Table 9 along with some of the possible methods of spplieation.

\section{MATERIALS AND METHODS OF APPLICATION}

\section{Material}

Cobalt base alloys

Ceramics (Alumina)

Tungsten Carbide

Titanium Carbide

Nickel Base Alloys

" "Liquid Metals Handbook"
Method of Application

Hardfacing by oxyacetylene or heliarc

Plasma spray (non-transfer arc)

Plasma spray or flame plate

Plasma spray

Spray welded

TABLE 9 


\subsubsection{Bearing Development Program (Cont.)}

\section{Discussion (Cont.)}

The cobalt base alloys for bearing applications can be supplied in three forms: castings, wrought or welding rod for hardfacing. Castings of alloys such as Haynes Stellite \#3 or Star "J" material are feasible; however, due to their low ductility, they are not considered practical for shrinking onto a shaft unless some special means of holding them is designed. Hardfacing or weld cladding, therefore, is considered more practical to investigate. In this group, the harder alloys, such as Haynes Stellite \#1 or \#1.2 would be tried first. The base metal for this application would probably be an austenitic stainless steel $(304,316)$ or a nickel base alloy (Inconel).

Alumina Ceramics applied to 304 stainless steel by plasma spray offer good bearing qualities. The plasma spray produces a dense coating which can later be ground to size. No difficulty is contemplated in coating $20^{\prime \prime} \mathrm{O}$. D. or I. D. surfaces.

Tungsten or titanium carbide have already been used with success as liquid metal bearings. The size in these applications were considerably smaller (3-5" diameter) than those required for future 20 " diameter designs. These bearings were solid pieces which, in a $20^{\prime \prime}$ diameter, is not practical. Therefore, plasma spray application appears to be a better method of obtaining carbide bearing surfaces. The base metal in this case would be austenitic stainless steel or nickel base alloys.

Nickel chrome boron alloys, such as the Colmonoy alloys or Haynes 40 alloys have been used in bearing applications and would be considered for this application. Spraywelded coatings also appear to have some chance of success. The base metal in this case could be austenitic stainless steel or a nickel base alloy.

Testing - Separate materials tests will be necessary to select the best combination or bearing materials to assure long life (minimum wear during start-up or low speed operation), dimensional stability, proper surface finish and hardness, and resistance to erosion and corrosion.

Test Apparatus - In order to check various materials and methods of applying or mounting these materials, a thermal shock and cycling test fixture is required. A sodium container capable of receiving $20^{\prime \prime}$ diameter cylinders and a hot sodium supply tank (with suitable gas piping so that sodium could be pushed from tank to tank) would be adequate for thermal shock and cycling testing.

Candidate bearings that have passed thermal tests would then be "load" and "stop-start" tested in the same or a similar bearing test stand as used for Task 1 testing. 


\subsubsection{Schedule and Cost}

It is estimated that this experimental program can be accomplished within an 18 month period at a cost of $\$ 308,000$ exclusive of the cost of bearing load determinations. The cost and schedule are shown on Figure 14. The cost of the bearing load determination is shown on the succeeding section on Pump Hydraulics Experiments in as much as it is planned to use the Pump Hydraulic models and test stand to determine the loads.

\subsection{PUMP HYDRAULIC DEVELOPMENT}

\subsubsection{Discussion of Problems}

A preliminary pump design study* based on the estimated hydraulic requirements for the pumps of a 1500 MWe sodium cooled nuclear power plant has pointed out the following major hydraulic problem areas.

1. The liquid metal plant system demands a pump which requires a low net positive suction head, and this is a primary consideration in the design of pump hydraulics. The low NPSH requirement will involve close attention to the design of the hydraulics, approach channels, impeller inlet areas, and the impeller blade inlet design. The fluid analytical techniques used to solve this problem in a water pump design will be applicable to liquid metals, and the resulting low NPSH design should be very similar to that for a water pump. To determine the degree of conservatism to be used in designing liquid metal hydraulics will require an understanding of the erosion and cavitation effects of liquid metals as compared to water. As a part of the design, it will be mandatory to search out any available information on this.

One design area which could be a problem of a special nature, is the extra large diameter shaft associated with the liquid metal pump. Because of the very high temperature liquid metal, extra shaft diameter is required to transmit power to the impeller. In the case of the double suction primary pump design, this extra shaft diameter could interfere with the impeller inlet design. A more detailed study of this situation could possible show that the effectiveness of a double suction impeller for low NPSH might be seriously limited for liquid metal pumps.

2. One of the critical areas in the design of liquid metal pumps is involved in the design and development of the hydrostatic radial bearing. Therefore, an important consideration in the design of the pump hydraulics will be the hydraulic radial thrust imposed by the pump. This will involve special design

*" Large Liquid Metal Pump Study - Hydraulic Design and Development," by H. G. Allen WAED Engineering Memorandum No. 3131., See Appendix 1. 
MONTHS FROM START OF CONTRACT

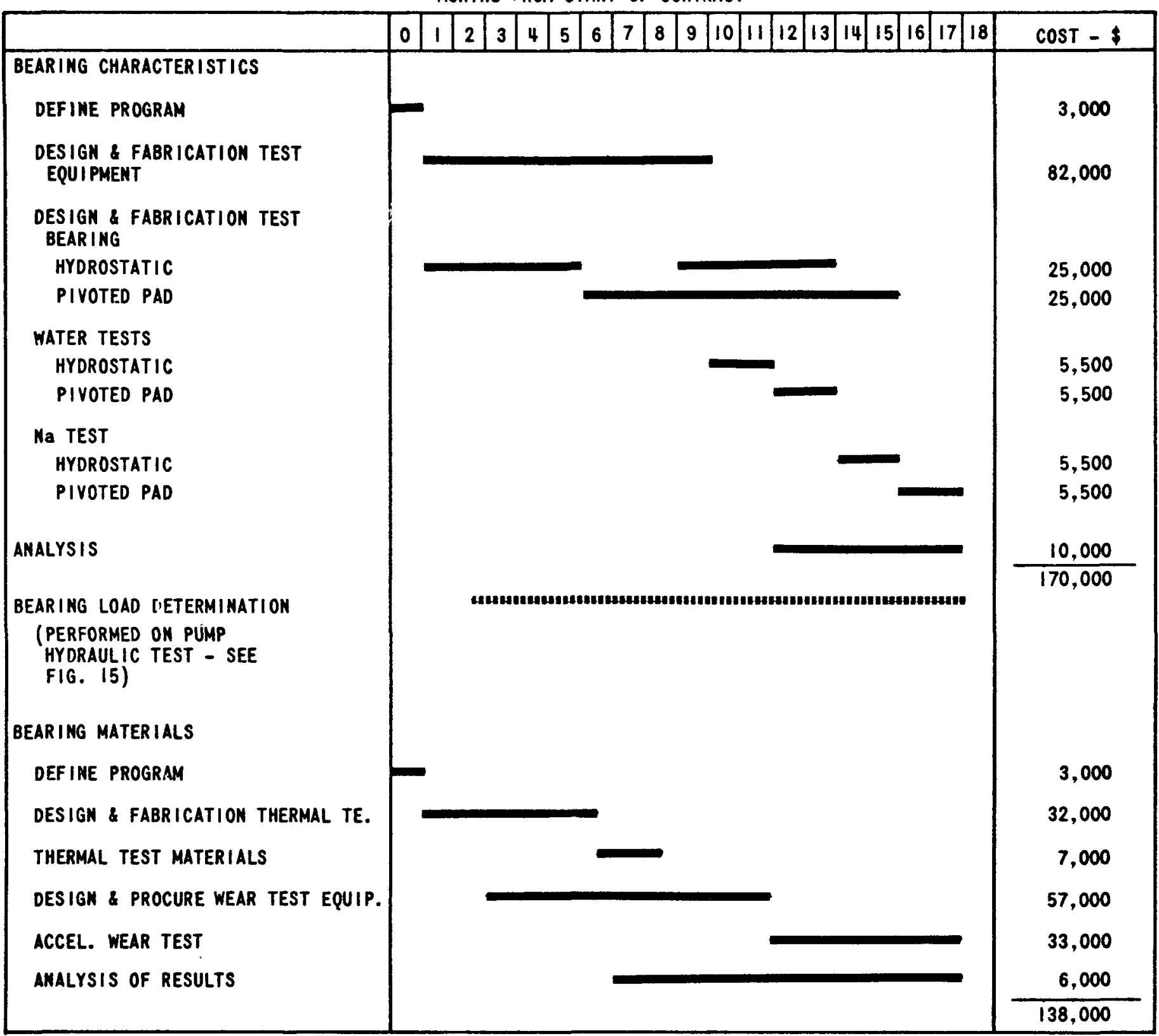

FIG. 14 BEARING DEVELOPMENT PROGRAM SCHEDULE AND COST 


\subsubsection{Discussion of Problems (Cont.)}

of the pump guide vanes and volutes to arrive at a proper design to obtain an acceptable radial thrust component.

Initial calculations have indicated that a double suction design has the best combination of size, weight, efficiency, and NPSH requirements for a primary coolant pump. However, model testing will be required to compare single and double suction types and to optimize the design from a performance and economy standpoint. Full scale testing is then necessary to confirm mechanical and thermal design under actual operating conditions. Radial thrust data must be obtained during model testing so that radial bearing loads can be factored into the pump type selection and to provide information on the bearing requirements so that bearings can be sized properly.

\subsubsection{Pump Development Program}

Object - The object of this program is ¿n develop and optimize pump hydraulics (approach channels, impeller inlet areas, impeller blade design, guide vanes, volutes, etc.) to obtain minimum radial thrust and required NPSH.

Testing - Model testing will be performed to verify design performance (head, flow, NPSH, etc.) while optimizing pump operation from radial thrust and low NPSH standpoints. These tests will determine which pump configuration offers the best combination of size, weight, NPSH, and load factors. The model tests will also confirm the vibration analysis.

Test Apparatus - Models $[1 / 3$ to $1 / 4$ size of initial development pumps, i.e., $5000-7000$ GPM will be made of at least two basic pump designs (single suction and double suction) and various diffuser and volute designs and modifications of these designs will be required. A fully instrumented water test loop will be needed along with the bearing load determination apparatus described in the Bearing Development section.

\subsubsection{Schedule and Cost}

It is estimated that the Phase I experimental program can be accomplished in 18 months at a cost of $\$ 231,000$ including the cost of the bearing load determination tests. The details of schedule and cost are shown on Figure 15. 
MONTHS FROM START OF CONTRACT

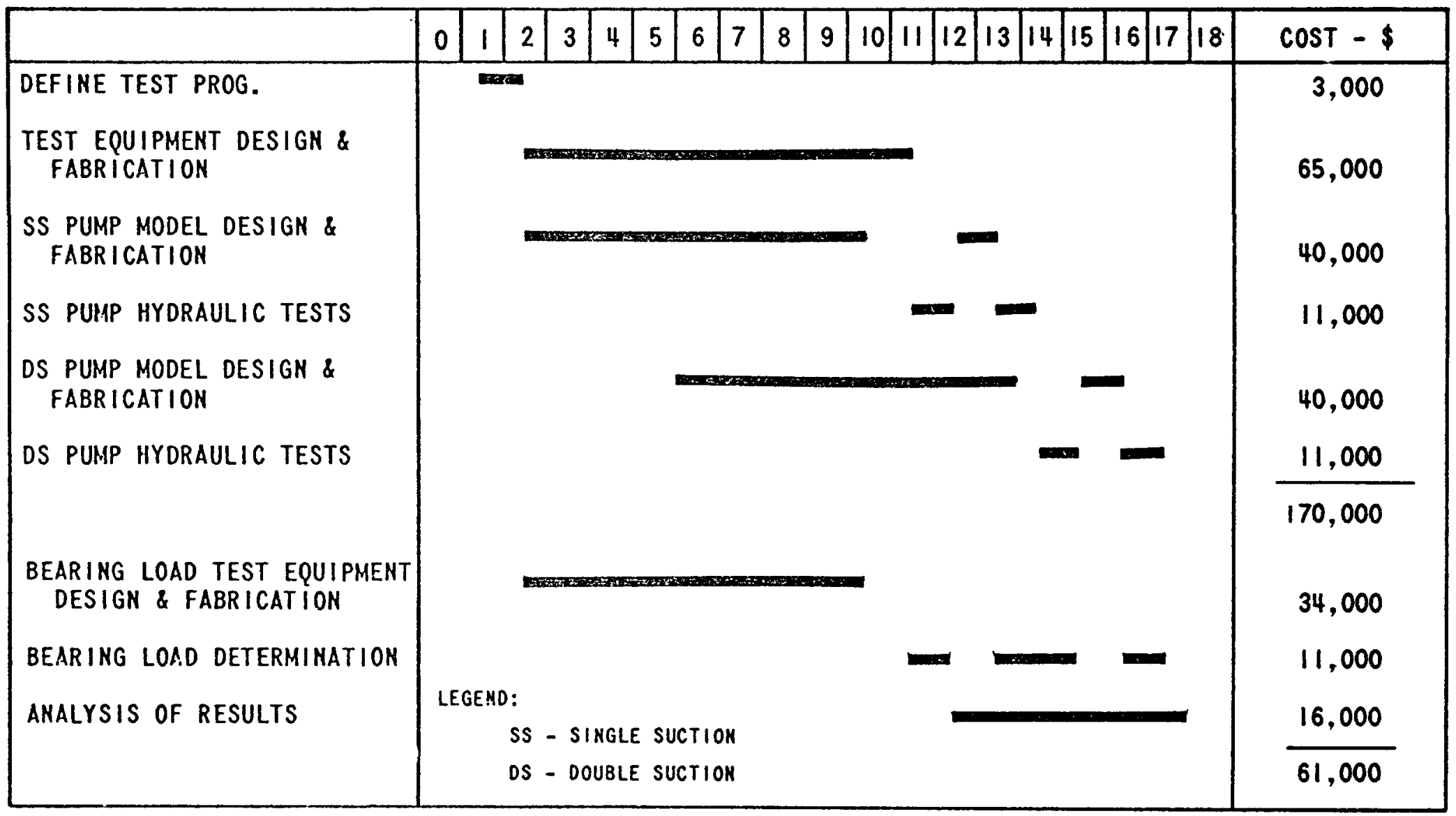

FIG. 15 PUMP HYDRAULIC DEVELOPMENT PROGRAM SCHEDULE AND COST 


\subsection{GENERAL}

In addition to the experimental development effort necessary to solve the major development problems, an additional effort is necessary in a number of ancillary areas in order to procede with the final design and fabrication of development pumps.

For the most part, work required in these ares will consist of analytical studies using existing engineering theory and practice. However, a certain amount of testing will be required to assure that application of current analytical methods and practice are adequate for prediction of performance of specific components of size and configurations required for the large pumps designs under consideration.

The problem areas to be investigated, the nature of the problems, and anticipated testing programs that will be required and an estimate of magnitude of effort are discussed in the succeeding sections.

\subsection{THERMAL STUDIES}

\subsection{1 $\underline{\text { Problems }}$}

The use of sodium in heat transfer systems results in higher thermal stresses in structural materials than would occur in conventional steam cooler systems, due to the low thermal resistance of sodium films. The effects of thermal transient conditions on pump components not only must be analyzed for stress reasons, but also to check the effects of thermal expansion on clearances and fits.

It is envisioned that the thermal barrier will be developed whereby a sufficient thermal drop can be realized such as to suppress the sodium temperature contained in the pump column to about $500-600 \mathrm{~F}$. This would favor bearing operational requirements, the materials of construction in this area, the sodium vapor problem general stress levels, and the overall thermal and thermal shock problems.

\subsubsection{Required Analysis and Testing}

A complete thermal stress analysis will be performed during the preliminary design of all components that contact the sodium, and all areas where close clearance must be maintained will be analyzed.

In order to get meaningful proof test data, full scale components would have to be tested. To do this, the entire assembled pump should be installed in a test loop for thermal testing. 


\subsubsection{Required Analysis and Testing (Cont.)}

Model thermal barriers may be built and tested prior to building a prototype pump. But, it is felt that a full size barrier for the prototype pump can be designed using existing theory, and more meaningful testing data will be obtained using the full scale pump test facility. Testing will involve placing thermocouples in strategic positions in and above the thermal barrier to check the effectiveness of the barrier for the suppression of temperatures in the lower bearing area.

\section{3 FLUID STABILITY STUDIES}

\subsubsection{Problems}

The free surface concept of pump design introduces problems of possible gas entrapment due to surface level instability and possible vortexing in the pump tank. A thorough understanding of the dynamic behavior of the liquid and vapor phase in this area under all conditions of pump operation is essential.

\subsection{2 nequired Analysis and Testing}

Models can be built to check fluid stability within the pump tank, but testing of a prototype pump will give more assurance of satisfactory operation under actual running conditions. Some of the tests which will need to be performed are:

(2) The effects of sodium level on pump performance, gas entrapment, vapor conditions, thermal considerations, etc. The gas and vapor mixture in the shaft seal vicinity will be thoroughly analyzed and its effect on seal condition and performance will be examined under various operation conditions.

(b) The effectiveness of the static sodium seal (see "Mechanical Considerations") at various discharge pressures and the effect on the level height.

(c) The ability of equalizing lines to limit sodium level in the pump tank.

(d) The effectiveness of pump drainage design.

(e) The reliability of the pump heaters to liquify the sodium prior to cold pump start-up.

(f) The effect of fluid whirl and vortexing on free surface height.

\subsection{DYNAMICS AND VIBRATION STUDIES}

\subsubsection{Problems}




\subsubsection{Problems (Cont.)}

The use of the free surface pump concept for primary pumps inherently requires the use of relatively long pump shafts which may operate at relatively high temperatures. These conditions make it manditory to investigate carefully system dynamics and vihrations.

\subsubsection{Required Analysis and Testing}

The required program will determine:

(a) Dynamics of rotating elements at all operating speeds.

(b) Torsional frequencies at all operating speeds.

(c) Vibration of external pump body and necessary mounting,

A preliminary analysis will be made based on expected operating requirements, so that a pump layout can be made. A model of the pump end and associated shaft will be constructed based on the theoretical dynamic analysis and be used to confirm the shaft analysis. The model can be the same model used in hydraulics tests.

\subsection{MECHANICAL STUDIES}

\subsection{1 $\underline{\text { Problems }}$}

Aside from the structural design, consideration must be given to assembly, disassembly and maintenance of the unit. It will be necessary to assure that the pump internals and shaft assembly can be inserted into or removed from the casing and pump tank (see Figure 16). With this design the entire inside assembly including the impeller, diffuser, thermal barrier, and lower bearing, shaft and baffling can be withdrawn from the pump tank and casing.

Some type of static seal will be required to minimize or prevent leakage between the pump tank and suction and discharge fit areas. Since there will be relative motion between these parts, due to thermal expansion, the seal will have to be a flexible or sliding seal.

Another mechanical design consideration will be to assure natural (gravity) drainàge of all pump parts when the system is to be emptied. This should not require any special development, but this will be a factor in the design of all components wet by sodium.

Pump mounting and support of the casing and piping is another major mechanical consideration.

\subsubsection{Static Seal Development and Testing}




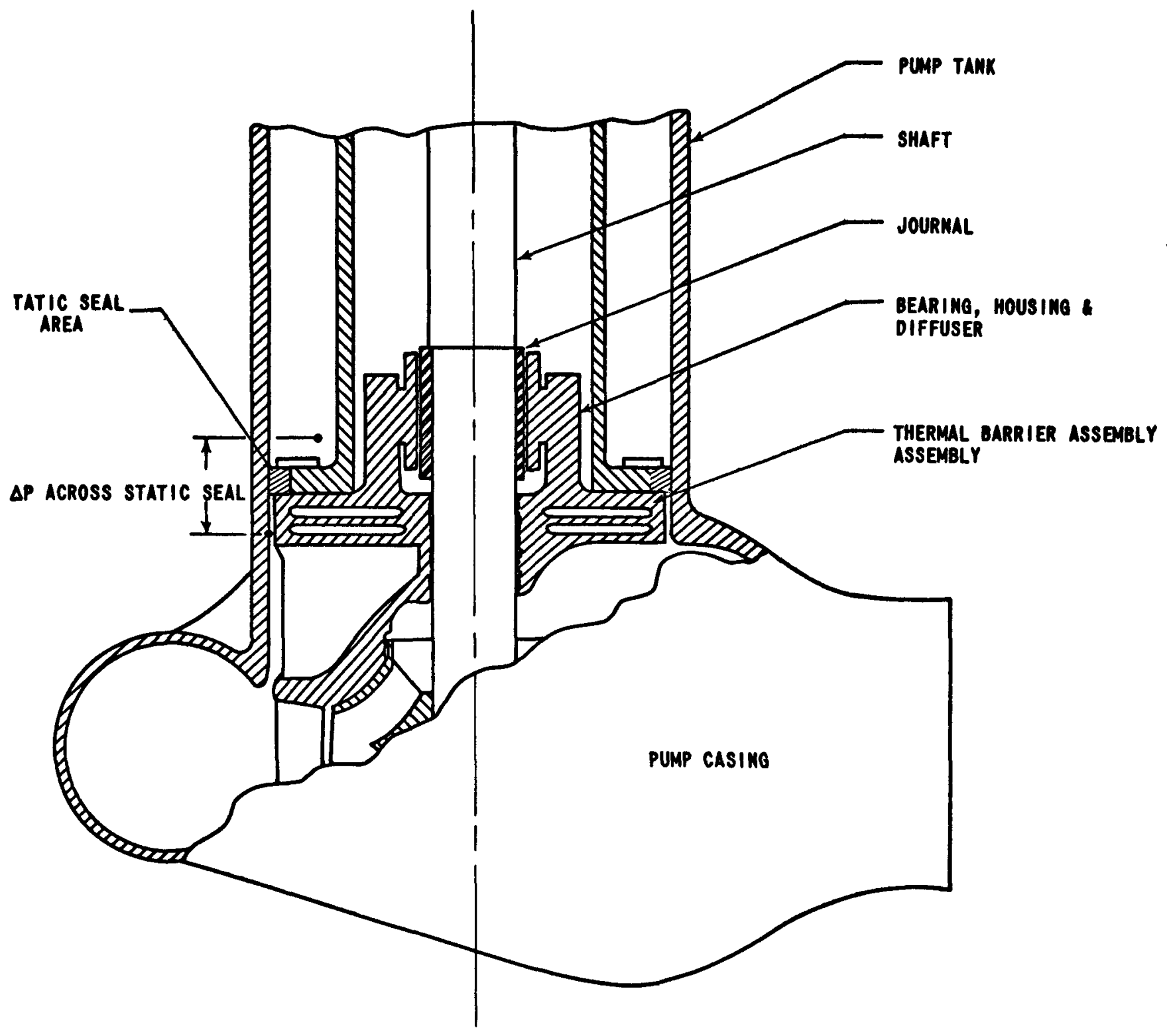

FIG. 16 LOWER END OF PUMP AND TANK SHOWING STATIC SEAL AREA

E.D. SK. 301858-8 


\subsubsection{Static Seal Development and Testing (Cont.)}

The object of this program will be to design, test, and prove a large diameter seal capable of limiting or eliminating sorium leakage between the pump tank I. D. and the lower bearing and thermal barrier assembly (Figure 16), caused by differential pressures in the pump tank and casing suction and discharge areas.

Apparatus - A sodium tank 4-6 ft. in diameter (equivalent to diameter of pump tank) and approximately one foot long will be needed to receive the seal. A means of detecting and varying the pressure across the seal will be required to check the leakage rates across the seal. Provision for heating the sodium to design operating temperatures $\left(1200^{\circ} \mathrm{F}\right)$ will be needed to check the effect of temperature on the seal.

Final testing of the pump in a full scale test facility should be performed to confirm drainage design and assembly and disassembly features. The static seal tests will also be confirmed during full scale pump testing, but it will be desirable to check some prospective seal designs.

\subsection{MATERIALS SELECTION}

\subsubsection{Problems}

For cost reasons, it appears desirable to use austenitic stainless steel (304) for the majority of pump structurals. No developmental testing appears necessary for the pump structurals, but the more critical areas such as bearings, seals and shafting may require testing.

\subsubsection{Required Analysis and Testing}

A survey of literature on existing materials would have to be performed for all pump components and initial material selection would be based on this. At the present time, bearing materials appear to be the only one which may require any extensive development.

The investigation and testing of bearing materials is included in the bearing development progrand dissussed in Section 8.0.

\subsection{ELECTRICAL DESTGN STUDIES}

\subsubsection{Problems}

Electrical Design Consideration - A design study ${ }^{\sharp /}$ of drive motors for large shaft sealed sodium pumps has revealed that would rotor induction motors appear to be the most suitable type of drive, and the production of these motors would

* "Sodium Pump Design Study-Drive Motor Btudy" by J.S. Wilhite, WAED E. M. \#3123 See Appendix 2 。 


\subsubsection{Problems (Cont.)}

not require any májor development. Motor design considerations will consist mostly of structural mounting and layout for vertical shaft sealed sodium pumps and development areas will be primarily mechanical and not electrical in nature, as long as speed regulation requirements are no greater than 4:1.

\subsubsection{Required Analysis and Testing}

The production of suitable would rotor motors will not entail any development in what might be considered unknown areas. Work in this area, unless another type of drive is selected will be limited to essentially standard design calculations for large special motors.

\subsection{INSTRUMENTATION AND CONTROL}

\subsubsection{Problems}

Instrumentation and control apparatus for the pump motor and auxiliaries will be necessary to assure that proper monitoring of temperatures, pressures, liquid level, vibration, etc. will be available on the prototype unit.

\subsubsection{Required Analysis and Testing}

A careful analysis must be made of experimental and developmental instrument requirements to achieve the required testing data both on models and prototypes. Apart from these requirements the only major developmental item anticipated is a high capacity sodium flow meter. While this latter item is not directly a part of pump development, it will be necessary for instrumentation of the pump testing facility.

\subsection{SCHEDULE AND COST}

The magnitude and distribution of the effort between the various programs described in this section that are estimated to be required for engineering analysis and preliminary engineering design of a development pump are shown on Figure 17.

In general, the costs estimated for work on these related problems, as shown in Figure 17, are based on engineering design and analysis only and experimental models and are not required or are provided for elsewhere in the program. The costs are the incremental costs that are additive to costs of other major programs.

Specifically, it is assumed that:

(1) The experimental tests of thermal barriers will be carried on the prototype pump in the Sodium Pump Test Facility. 
MONTHS FROM START OF CONTRACT

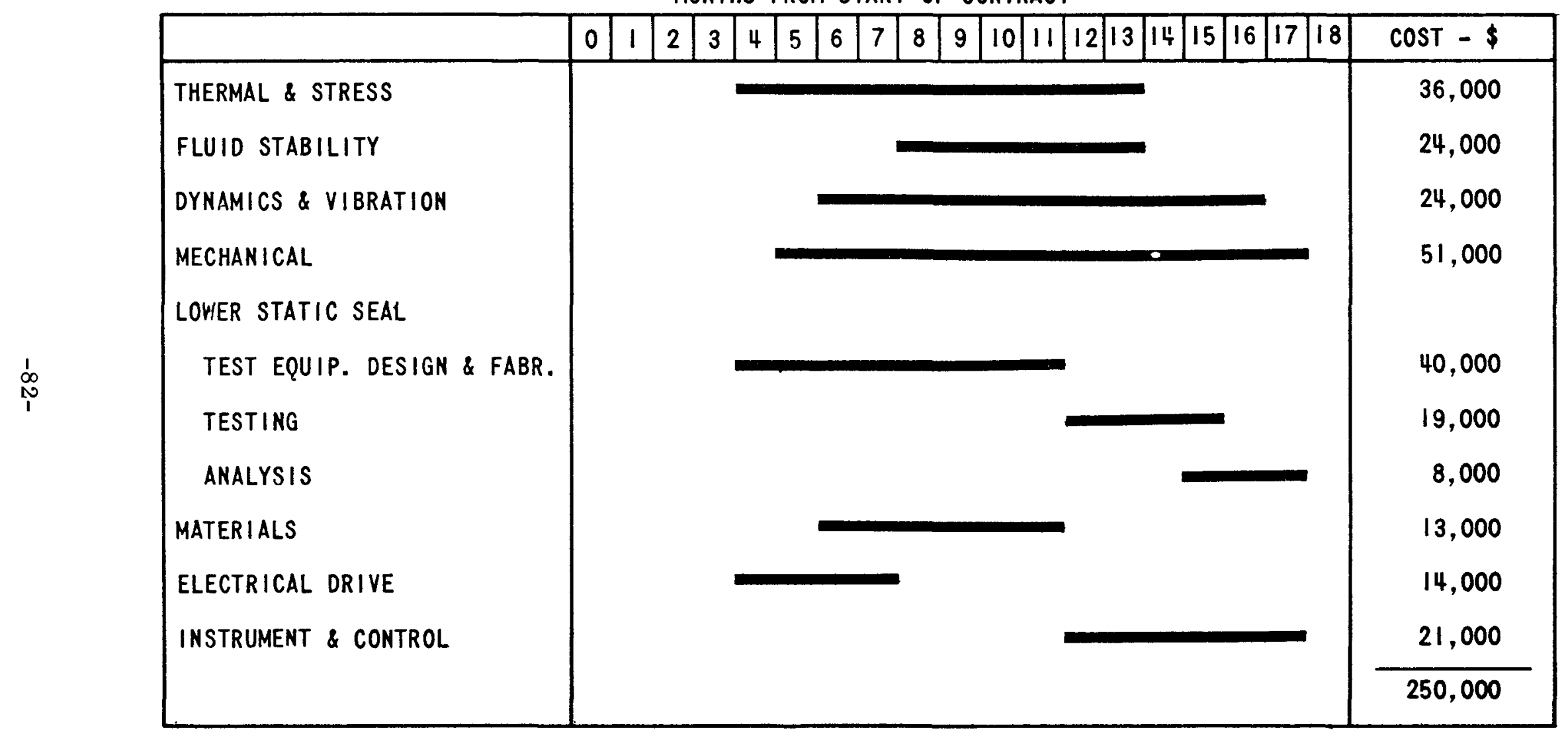

FIG. 17 ENGINEERING ANALYSIS OF ANCILLIARY PROBLEMS 


\subsection{SCHEDULE AND COST (CONT.)}

(2) Model tests for dynamic and vibration analysis Section 9.4.2 will be performed on the hydraulic test model Section 8.3.2.

(3) The fluid stability tests will be performed on the prototype in the Sodium Pump Test Facility.

The one exception to this rule is in conjunction with the development and testing of a lower static seal. In this instance, testing prior to completion of the pump prototype is desirable and will entail equipment not available under other major programs. In this case the cost of test apparatus is included. 
10.0 SODIUM PUMP TEST FACILITY REQUIREMENTS

10.1 GENERAL

Experimental facilities for testing and demonstrating performance are a necessary and obvious adjunct to the successful development of large sodium pumps. The size, scope and requirements of such facilities are a direct outgrowth of the estimated pump requirements and the related development program. The pump requirements and related development program of the earlier sections of this study have been reviewed in order to develop the following design requirements and criteria of a single large multipurpose facility to meet the specific needs of the large sodium pump program.

In the past, the testing, development, and demonstration of sodium pumps for reactor power applications has been accomplished by building necessary facilities as required for testing a specific pump under development in conjunction with the related reactor. Such facilities provided essentially for testing steady state design performance and generally operated isothermally over a series of design temperatures, at flow and head conditions of the reactor under design. Such facilities provided valuable demonstration of general pump performance. These test programs, in conjunction with prior water model testing and additional flow testing in the actual reactor system, have resulted in pumps that appear satisfactory for current requirements. The developing emphasis on liquid metal cooled reactors coupled with the estimated substantial increase in pump size, suggests that further extension of this philosophy of separate facilities for each reactor will bacome prohibitively expensive and that the overall requirements of the liquid metal reactor program and related pump development program for the next decade can be better served by a single multipurpose sodium pump test facility.

\subsection{FUNCTION}

The primary function of the Sodium Pump Test Facility (SPTF) is to perform such testing as is necessary to the development of large sodium pumps and to demonstrate the performance and operation of such pumps for nuclear power plant service under simulated reactor operating conditions.

Concomitantly, the facility will provide a large flow loop which will permit demonstration of related flow measurement and control devices and will in itself provide additional information on the design and operation of large sodium piping systems.

\subsection{TEST CAPABILITIES}

\subsubsection{General Considerations}

The facility must provide the necessary basic fixed equipment and instrumentation 


\subsubsection{General Considerations (Cont.)}

necessary to carry out a flexible and comprehensive test program necessary to the development of large sodium pumps of various types that will be developed under the overall pump development program. The facility should also provide adequate provision for the addition of specialitest instrumentation and for adaption to various pump types and pump drive requirements. Both steady state and transient tests in the following categories are deemed to be necessary and desirable on the basis of future pump requirements and proposed programs of development as noted in previous sections. The major categories include:

(1) Steady State Hydraulic and Electrical Characteristics

(2) Hydraulic Transients

(3) Thermal Transients

(4) Flow Control - Speed Control Response Rates

(5) Component Evaluation

(6) Accelerated Life Tests

\subsubsection{Steady State Hydraulic Tests}

These tests are primarily for demonstrating pump operation over the specified design range and for determining by actual test, the pump characteristics. Provision shall be made for measuring head developed, flow, input power and efficiency over the full design range of pump speed, pumping temperature and NPSH.

\subsubsection{Hydraulic Transients}

The practical demonstration of pumps for reactor application require the determination of operating characteristics and behavior under a variety of nonsteady state hydraulic conditions. It will be necessary to test, measure and evaluate the performance under conditions of variable liquid level in the pump casing, variable cover gas pressure and to determine the interactions that occur between parallel loop operation with common suctions, discharges or both.

\subsubsection{Thermal Transients}

In an operating reactor system, the circulating pumps and associated piping will be subject to a series of thermal transients that result either from usual operating conditions or from abnormal conditions of equipment failure. Typical conditions are usual power changes not immediately compensated by adequate flow regulation and reactor shutdown. More extreme conditions result from reactor scrams without 


\section{3.4 Thermal Transients (Cont.)}

compensative flow control or from failure of secondary or primary pumps. It is important to demonstrate the adequacy of pump and loop design to withstand such changes. An important part of the testing requirement will entail inducing transients on pumps during tests to observe their performance and design oharacteriatics. The facility will require a method of introducing thermal transients of varying severity and duration in both the upward and downward direction.

\subsubsection{Flow Control With Pumps}

In sodium cooled reactor systems it is usual to operate at essentially constant coolant temperatures and to vary the coolant flow in accordance with power changes. In the past, both flow control valves and variable speed pumps or combinations of these have been used to control coolant flow. Flow control valves have in the past been a source of some considerable difficulty for this service. With the advent of larger flows and piping it is anticipated that control of flow by means of pumps will assume even greater importance. It will, therefore, be necessary to provide for testing and evaluating performance of a variety of pump variable speed drives with respect to flow control response rate and accuracy of flow control.

\subsubsection{Component Evaluation}

The overall pump assembly consists of a considerable number of discrete mechanical components and subassemblies including bearings, shaft seals, shafts, casing, lower end static seals, thermal barriers, etc. These components will have been the subject of varying amounts of development, model testing and analysis during the prototype design and development as noted in the development program outlined in Sections 8 and 9. An extensive testing program will be required in the SPTF to confirm the design analysis and to provide improved design and performance data of these components as finally incorporated in the prototype designs.

Among the many tests that might arise, the following are typical and recognizable at this stage:

(1) Determination of shaft seal leakage

(2) Confirmation of vibration analysis for rotating machinery

(3) Confirmation and measurement of bearing loads under operating conditions

(4) Efficiency of thermal barrier design

(5) Extent and conditions for cover gas entrainment 


\subsubsection{Component Evaluation (Cont.)}

(6) Effectiveness of lower static seal design

To a large extent it is anticipated that this information can be obtained concurrent with other testing programs by the use of adequate test instrumentation.

\subsubsection{Life Tests and Accelerated Wear Tests}

The facility shall provide for determining some measure of pump reliability and maintenance free lifetime by operation for extended periods at any of the above test conditions or combinations of same. Accelerated wear tests can to some extent be obtained by means of start-stop tests and through the use of a large number of transient test cycles.

\subsection{OPERATING RANGE FOR SODIUM PUMP TEST FACILITY}

The key parameters that establish the design criteria for the SPTF are:

(1) Flow

(2) Coolant Temperature

(3) System Head

(4) Power

These parameters directly reflect the estimate of future pump requirements as discussed and selected in Section 4 and summarized in Table 4. A consideration of these pump requirements and the testing capability required established the following range of operation for the SPTF.

Flow

Maximum total flow of coolant for the facility of 120,000 GPM.

Temperature

Steady state operation at all sodium temperatures from $400^{\circ} \mathrm{F}$ to $1200^{\circ} \mathrm{F}$.

Thermal transient operation over range of $400^{\circ} \mathrm{F}$ to $1200^{\circ} \mathrm{F}$ capable of maintaining $10^{\circ} \mathrm{F} / \mathrm{sec}$. for periods up to $30 \mathrm{sec}$. and for average rates of at least $4 \mathrm{~F} / \mathrm{sec}$. for periods up to 3 minutes.

\section{System Head}

Maintain system head of up to $350 \mathrm{ft}$. of $\mathrm{Na}$ over range of flows. Maintain net positive suction head over range of 1 psig to $50 \mathrm{psig}$. 


\subsection{OPERATING RANGE FOR SODIUM PUMP TEST FACILITY (CONT.)}

Power

Provide installed electrical power supply for pump motor drives to a maximum of $10 \mathrm{MW}$.

\subsection{GENERAL CONSIDERATIONS}

\subsubsection{Flow Loops}

In order to attain the maximum flow capacity of 120,000 GPM and still maintain reasonable equipment sizes and operating flexibility at less than this maximum flow, the facility will be designed as two identical flow loops of 60,000 GPM each. This arrangement permits the simutaneous independent operation of two pumps with individual ratings of up to $60,000 \mathrm{GPM}$ or with suitable cross-connections the parallel operation of two pumps with individual ratings of up to $60,000 \mathrm{GPM}$. Operation of a single pump with capacity in range of 60,000 to 120,000 GPM is attainable by connecting such large pumps to the two loops operating as parallel flow paths.

\subsubsection{Temperature Adjustment}

Independent controllable sodium - air heat changers will be in each loop to reject pump heat and maintain essentially isothermal conditions in the test loop. The coolers will be designed to reject full pump heating load at lowest fluid temperature, thus providing some excess heat rejection capacity. The pump heat input and these coolers provide for adjustment of temperature level in the test loop.

\subsubsection{Transient Simulation}

The high rate short period thermal transients will be induced in a loop initially operating at temperature $T_{1}$ by a feed and bleed system from a reservoir (alternate loop and tankage) containing a supply of sodium at a substantially different temperature $T_{2}$. Proper selection of initial temperature and control of feed and bleed rates permits controlled simulation of a wide range of transient conditions without the need for excessive heat exchanger capacity or heat input.

Low rate, long period thermal transients may be obtained by control of the sodium-air heat exchangers.

\subsubsection{Flow Measurement}

Flow measurement devices will be provided in each loop.

\subsubsection{Flow Resistance}




\subsubsection{Flow Resistance (Cont.)}

Variable flow resistance in the form of some type of valve will be provided in each loop.

\subsubsection{Tanks}

Each flow loop will be provided with a supply tank to function as a surge tank and to provide the necessary heat supply and heat sink reservoirs for thermal transient tests. In addition, a common drain tank will be provided.

\subsubsection{Pump Test Bed}

The experimental nature of the facility requires that the pump test bed structure and arrangement be designed to permit a wide latitude in pump sizes and geometries. The necessary structures should be arranged to permit installation of two pumps at a time, one feeding each test loop and provide for the installation of a single larger pump connected to both loops as parallel flow circuits. Adequate room should be provided to permit replacement and modification of pump inlet and discharge piping from the pump casing to the fixed loops for varying sizes and varying nozzle orientations.

\subsubsection{Supporting Systems and Services}

In addition to the major design requirements noted above, a considerable number of supporting systems and services will be required in order to operate the loop and adequately carry out the experimental functions. These include:

(a) A sodium loading facility for initial loading and make-up of coolant.

(b) A sodium purification system including provision for both hot and cold trapping to maintain desired coolant purity.

(c) An inert gas handling system for supplying, storing and controlling inert cover gas necessary for precluding oxygen entrance into the system and in providing a variable cover gas pressure for adjustment of NPSH on the pumps.

(d) A secondary cooling system to provide for freeze seal valves etc.

(e) An auxiliary heating system to provide necessary heat input for preheating system, heat tracing as required, and maintaining temperature in storage tanks.

(f) Component handling facilities for installation, removal and transport of pumps between test bed and inspection area.

(g) Component decontamination and inspection facilities for inspection, partial 
10.5.8 Supporting Systems and Services (Cont.)

disassembly and limited maintenance on experimental pumps.

(h) A safety system for detection and alarm, of leaks and for the control of fire. 


\subsection{PRE LIMINARY CONCE PT OF SODIUM TEST FACILIT'Y}

A preliminary concept of the Sodium Pump Test Facility which embodies the major considerations and requirements is presented in a simplified flow diagram Figure 18 and two conceptual drawings of the facility Figure 19 and Figure 20.

A more detailed evaluation of the concept including necessary revisions, modifications and expansion based on an engineering design study and the preparation of a cost estimate will be carried out in Phase II of the contract work which will be subsequently reported. 


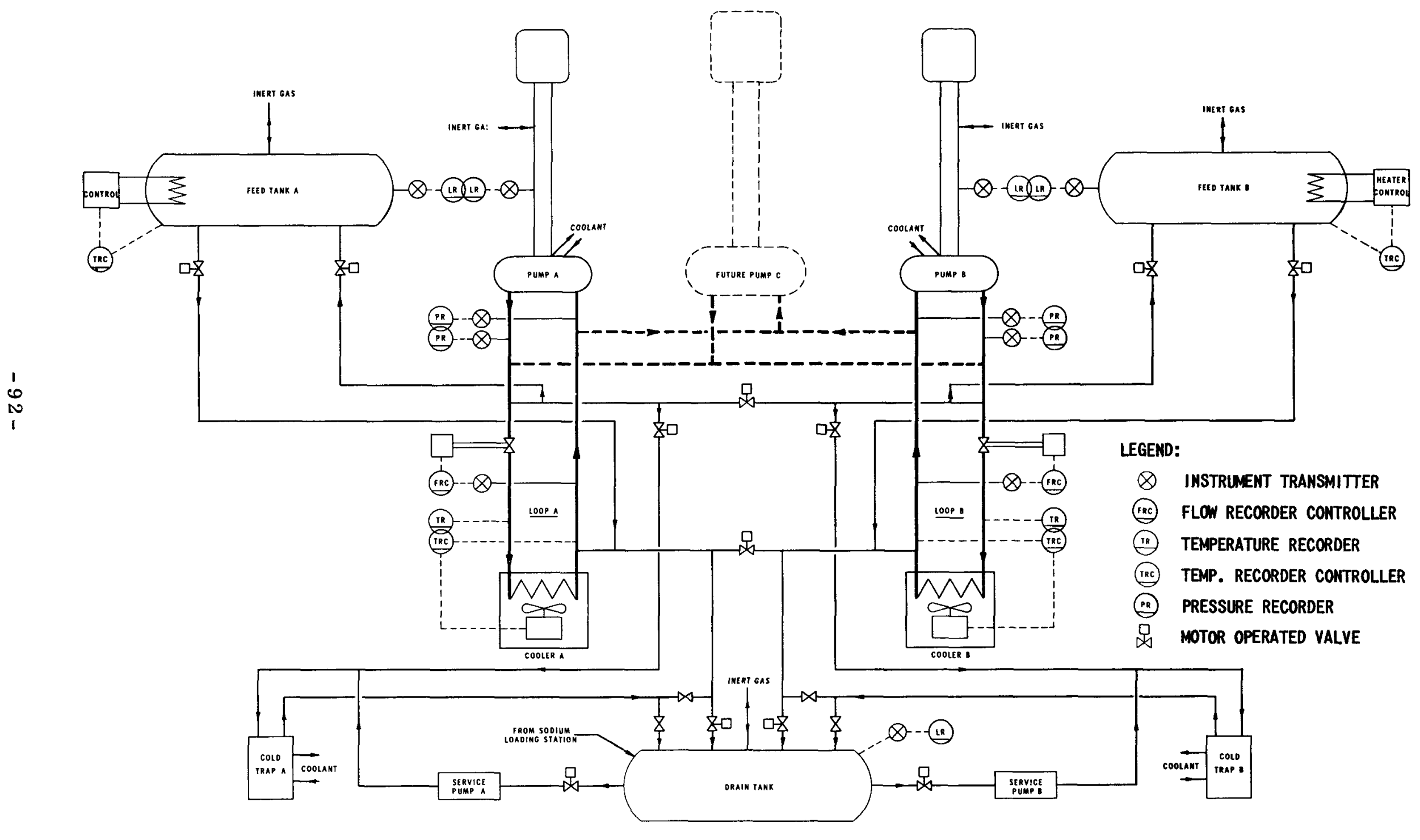

FIG. 18 PRELIMINARY SPTF FLOW DIAGRAM 


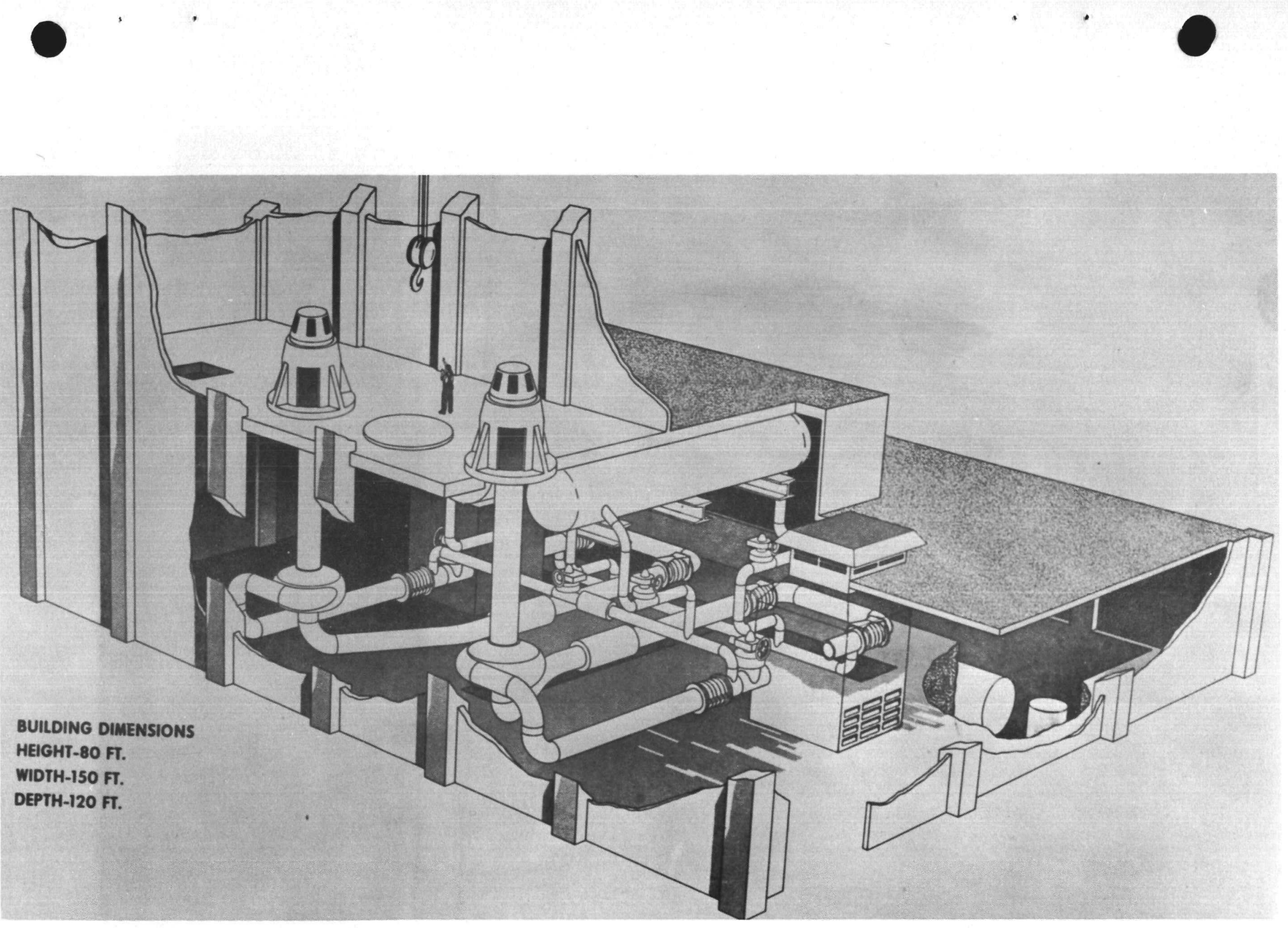

FIG. 19 ARTISTS CONCEPT OF SPTF TWO PUMP ARRANGEMENT 


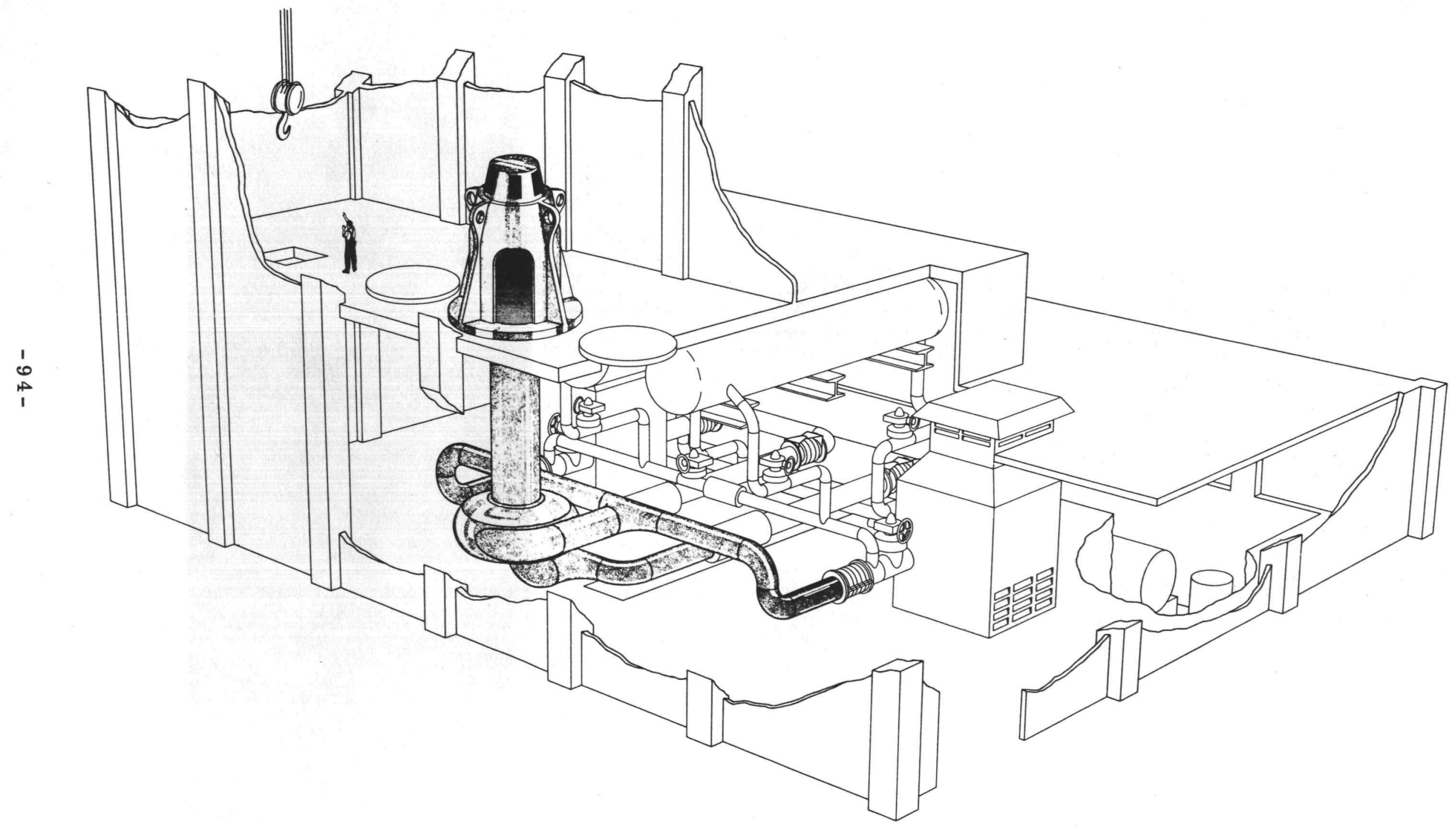

FIG. 20 ARTISTS CONCEPT OF SPTF LARGE SINGLE PUMP ARRANGEMNT 
APPENDLX 1 WAED EM 3131

WAPD LARGE LIQUID METAL PUMP STUDY

HYDRAULICS DESIGN AND DEVELOPMENT

By

H. G。Allen

May 23, 1963

S.0. IDII21

ENGINEERING MEMORANDUM NO. 3131

WESTINGHOUSE ELECTRIC CORPORATION

ATOMTC EQUIPMENT DIVISION

CHESWICK, PENNSYLVANIA 
TABLE OF CONTENTS

B TABULATION OF DATA COVERING PRIMARY AND SECONDARY FULI JAPACITY PUMP DESIGNS ..... 1

C DESIGN SELECTION CRITLRTA . . . . . . . 2

D PRIMARY PUMP DESIGN SELECTION. . ...... 3

E SECONDARY PUMP DESIGN SELECTION. . . . . . 5

F HALF-CAPACITY HYDRAULIC DESIGNS FOR PRIMARY AND SECONDARY UNITS. . . . . . . . . 6

G SPECIAL HYDRAULICS PROBLEM AREAS INVOLVED IN THE DESIGN AND DEVELORMENT OF A PUMP FOR

HIGH TEMPERATURE LIQUID MEIALS . . . . . 7

H TABULATION OF CURVES

Curve D447 - Hydrauizio Efficiency and Relative

Volute Weight VS. Pump Specific Speed. . . . 9

Curve D448 - Design Data VS. Pump Speed -

Primary Liquid Metal Pumps . . . . . . . 10

Curve D449 - Design Data VS. Pump Speed -

Secondary Liquid Metal Pumpe . . . . . . . 11 
E.M. \#3131

A. INTRODUCTION

The following report covers the hydraulic portion of the Large Liquid Metal Pump Study conducted by WAED on shop order $1 D 1121$ for WAPD. Hydraulic design selections are offered for two separate applications ow (1) A primary pump to handle $32 \times 10^{6}$ lbs. per hour of $1200^{\circ} \mathrm{F}$ radioactive sodium at $300 \mathrm{ft}$ total head, and (2) A secondary pump suitable for $32 \times 20^{6} \mathrm{lbs}$. per hour of $700^{\circ} \mathrm{F}$ nonradioactive sodium at $150 \mathrm{ft}$ total head. The pump driving motor is to be mounted on top of the shield deck. The pump volute is to be mounted in the piping system below the shield with the pump rotor suspended from the driver and shafting through an interconnecting vertical column between the driver and the pump volute. A static height of liquid sodium is to be maintalned with the free liquid surface at some intermediate polnt within the column. An Inert gas cover is to be provided over the free surface with sufficient pressure to assure required NPSH to the pump. This preliminary study indicates that the best hydraulic design selections for these two pumping applications would be a double suction design driven at $890 R_{0} P_{0} M_{\circ}$ for the primary unit, and a single suction hydraulic design driven at $710 R_{0} P_{0} M_{0}$ for the secnndary unit.

B. TABULATION OF DATA COVERING PRIMARY AND SECONDARY FULC CAPACITY POMP DESIGNS

The following is a tabulation of some of the leading particulars of these two hydraulic designs.

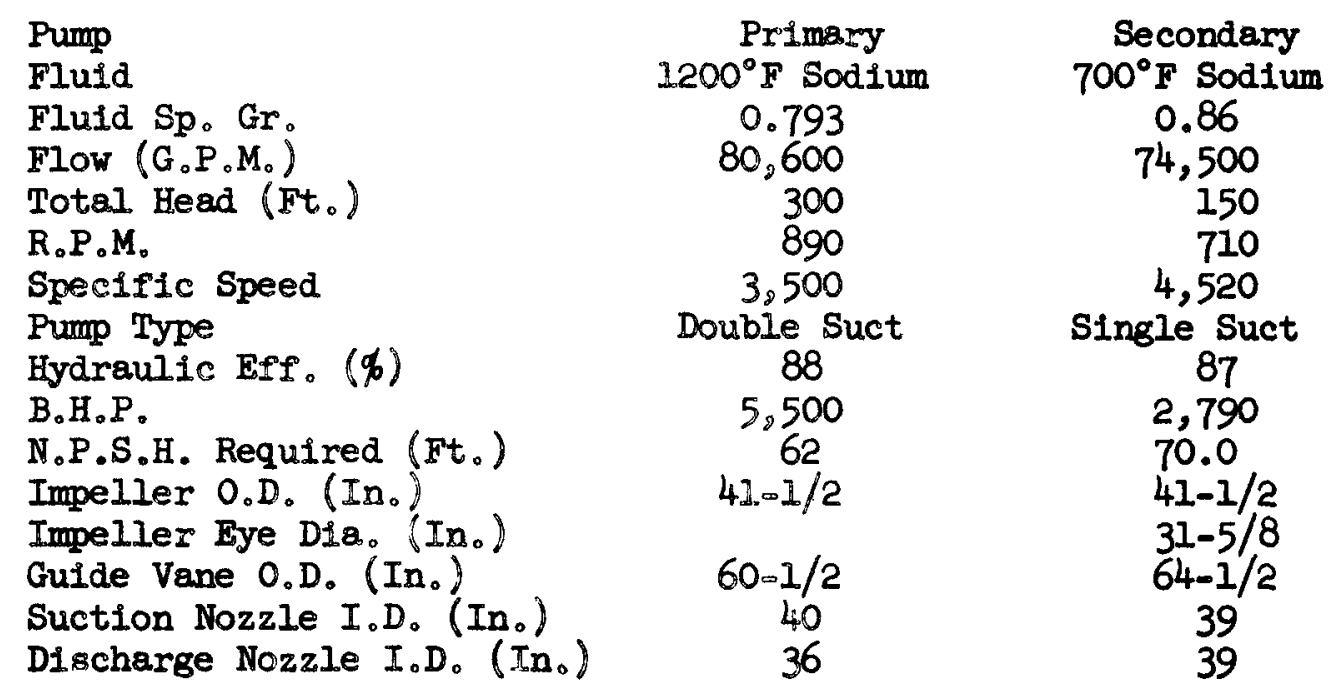




\section{DESIGN SELECTION CRITERIA}

The principal criteria in making these hydraulic selections are:

(1) Economy in weight。

(2) H1gh level hydraulic performance.

(3) Low net positive suction head requirements.

Curve $D 447$ has been constructed to show how the pump hydraulic efficiency and volute weight will vary with pump specific speed. The hydraulic efficiency value is the best efficiency occurring at the pump design flow point. The weight curve shows the relative volute weight for pumps of various specific speed designs to meet a particular head - flow point. The pump specific speed (Ns) $=\frac{\mathrm{N} \sqrt{\mathrm{Q}}}{3 / 4}$

$$
\text { where, } \begin{aligned}
N & =\text { pump speed in } R_{0} P . M \\
Q & =\text { pump design flow in G.P.M. } \\
H & =\text { pump design total head in feet. }
\end{aligned}
$$

Specific speed number is a conventent way of comparing pump designs where the pump similarity laws are used in computing pump size and performance.

Some important conclusions can be drawn from Curve D447. This curve indicates that a 2500 specific speed design is an optimum for maximum hydraulic efficlency, whereas 5200 speciflc speed is an optimum for minimum volute weight. For very large pumps where pump size is a critical consideration, as is the case in this pump study, Curve 0447 illustrates that pumps within the 3000 to 4500 specific speed range would appear to be the best selection. Pump weight becomes excessive at specific speeds less than 3000 ; and for specific speeds greater than 4500 , the hydraulic efficiency reduction becomes excessive.

For most pump applications the greatest proportion of the pump weight is concentrated in the pump volute, so the pump with the lightest welght volute will, in general, be the most economical selection. However, as will be pointed out later, this mule is not absolutely correct for the pump designs considered in this study.

The remaining consideration involved in selecting a pump design -the low net positive suction head requirement -- is not covered by the foregoing Curve D447. As mentioned in the introduction to this hydraulic study, a static height of liquid sodium will be provided in the vertical pump column with a gas cover sufficiently pressurized to provide the required NPSH. However, to minimize the gas seal design problems, it would be advantageous to provide a pump design which 
would be compatible with a gas cover requiring only a low pressure. The NPSH made available to the pump can be calculated as follows:

$$
\begin{aligned}
\text { NPSH }_{\text {avall. }}= & \mathrm{H}_{\mathrm{a}}+\mathrm{H}_{\mathrm{P}}+\mathrm{H}_{\mathrm{S}}-\mathrm{H}_{\mathrm{V}} \\
\text { where, } \mathrm{H}_{\mathrm{a}}= & \text { atmospheric pressure } \\
\mathrm{H}_{\mathrm{P}}= & \text { gage pressure of gas cover } \\
\mathrm{H}_{\mathrm{S}}= & \text { static height of fluid column above the } \\
& \text { impeller centerline } \\
\mathrm{H}_{V}= & \text { fluid vapor pressure }
\end{aligned}
$$

The units for the above is in feet of fluid being pumped.

With the use of the above formula, it can be shown that about $75 \mathrm{ft}$. NPSH is made available to the primary pump handling $1200^{\circ} \mathrm{F}$ sodium under a $6 \mathrm{ft}$. static fluid height in the vertical column and a $10 \mathrm{psig}$ gas cover pressure. The secondary pump handling $700^{\circ} \mathrm{F}$ sodium under the same static fluid height and gas cover pressure would have $72 \mathrm{ft}$. NPSH available. Based on this line of reasoning, the following NPSH values have been selected as a design condition for the pump designs being considered in this study:

$$
\begin{aligned}
& \text { Primary Pump - Required NPSH ₹ } 75 \mathrm{ft} \text {. } \\
& \text { Secondary Pump - Required NPSH } ₹ 72 \mathrm{ft} \text {. }
\end{aligned}
$$

\section{PRIMARY PUMP DESIGN SELECTION}

Curve D 448 has been prepared for the primary liquid metal pumps with the head-flow parameters set at $300 \mathrm{ft}$, and 80,600 G.P.M. This curve shows how some of the other principal purmp parameters and dimensions w11l change with the pump speed selection. The speed range covered is from 550 to 1200 R.P.M. with a corresponding specific speed range from 2150 to 4740 . The solid line curves are for a single suction pump design - - the dashed line curves are for the double suction design where it differs from the single suction design. In general, this curve shows a rather rapid dimensional and weight increase with decreased pump design speed, and the required NPSH decreases with decreased pump speed.

In comparing the single suction design with the double suction pump, this Curve $D 448$ shows that the main differences are in volute weight, NPSH requirements and suction nozzle size. An explanation for this follows: 
(a) Approximate weight calculations indicate $30 \%$ greater volute weight for the double suction pump. This is the additional weight required for the larger diameter side suction nozzle furnished with the double suction design.

(b) Pump NPSH requirements can be approximately computed by the following formula:

$$
\begin{aligned}
& \text { Single Suction } \\
& \operatorname{NPSH}^{3 / 4}=\frac{N \sqrt{Q}}{S_{N}} \\
& \text { Double Suction } \\
& \mathrm{NPSH}^{3 / 4}=\frac{\mathrm{N} \sqrt{Q / 2}}{\mathrm{~S}_{\mathrm{N}}} \\
& \text { where, } N=\text { pump speed in R.P.M. } \\
& Q=\text { pump design flow in G.P.M. } \\
& S_{\mathrm{N}}=\text { suction specific speed }=8000 \text {. }
\end{aligned}
$$
pump design.

\section{Curve $D 448$ can be entered at four speed selection points:}

(1) 1180 R.P.M. - Induction motor speed for 6 poles, 60 cps

(2) 890 R.P.M. - Induction motor speed for 8 poles, 60 cps

(3) 710 R.P.M. - Induction motor speed for 10 poles, 60 cps

(4) 590 R.P.M. - Induction motor speed for 12 poles, 60 cps

Examining Curve D448 at the above four speed values, the following single suction and double suction pump designs are found to be the most economical selections for the required NPSH $₹ 75$ feet:

$\begin{array}{lcc}\text { Pump Design } & \text { Single Suct. } & \text { Double Suct. } \\ \text { Speed (R.P.M.) } & 710 & 890 \\ \text { Relative Volute Wt. } & 1.28 & 1.44 \\ \text { Req'd. NPSH (Ft.) } & 74 & 63 \\ \text { Guide Vane O.D. (In.) } & 71-1 / 2 & 60-1 / 2\end{array}$


E.M. \#3131

At first glance, the above would appear to indicate that the 710 R.P.M., single suction hydraulic design with 1.28 relative volute welight would be the more economical selection of the two designs. However, the internal parts of the double suction design are smaller (60-1/2 in. dia. guide vane as compared to $71-1 / 2$ in. for the single suction design). A closer study would probebly show that the 12\% greater volute weight for the double suction design is more than offset by the greater vertical pump column diameter required for the withdrawal of the larger diameter single suction guide vane. This fact, along with the somewhat better NPSH requirement obtainable with the double suction design is responsible for the 890 R.P.M. double suction selection for the primary liquid metal pump as previously outlined in this report.

\section{E. SECONDARY PUMP DESIGN SELECTION}

Curve D449 has been prepared for the secondary liquid metal pumps which are rated at $150 \mathrm{ft}$. total head and 74,500 G.P.M. This curve is constructed in the same manner as primary pump data curve D448. Curve D449 covers a speed range of 550 to 1200 R.P.M. With a corresponding specific speed range or 3500 to 7650 . The most apparent difference between this Curve $D 449$ and zurve $D 448$ is in the shape of the relative weight characteristic. D449 shows a relatively flat weight characteristic, so that a pump selection can be made over a wide range of speeds with little effect in the pump volute weight. This is to be expected, because the secondary pump hydraulic conditions fit into a higher specific speed range than for the primary pump conditions.

The double suction pump design is not an attractive offering for the secondary pump application. Because of the flat relative welght characteristic shown on Curve D449, the increased speed obtainable with the double suction design will not result in a general weight reduction to offset the increased volute weight required for the double suction design. Also, the pump total head required for the secondary pump is only 150 feet. Rotor parts of about the same diameter as used for the primary pumps can meet this $150 \mathrm{ft}$. total head at a relatively lower speed.

Curve D449 shows that the economical pump selection for a required NPSH $₹ 72$ feet is a single suction design operating at 710 R.P.M. Some of the principal design details for this pump are included in a tabulation in a previous section of this report. 
F. HALF-CAPACITY HYDRAULIC DESIGNS FOR PRIMARY AND SECONDARY UNITS

During the progress of this hydraulic design study, it was requested that attention be given to half-capacity liquid metal pump designs. The following tabulation lists some of the leading particulars of these hydraulic designs. These pumps are a counterpart to the above fullcapacity designs. The principal aim here was to show how the halfcapacity designs will differ from the full-capacity pumps, maintaining approximately the same NPSH performance.

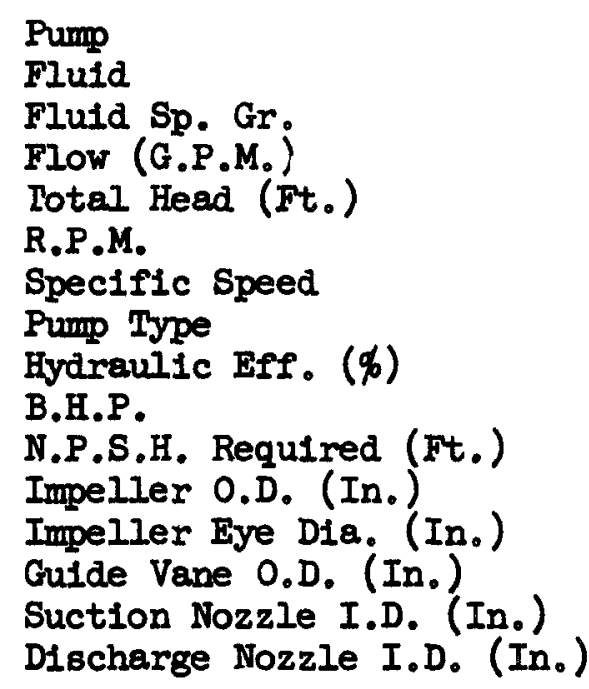

Primary
$1200^{\circ}$ F Sodium
0.793
40,300
300
1,180
3,290
Double Suct.
88
2,755
60
$30-1 / 2$
$44-1 / 2$
$30-1 / 2$
26

Secondery
$700^{\circ}$ Podium
0.86
37,250
150
890
4,000
S1ngle Suct.
87
1,395
63
$30-3 / 4$
24
$50-3 / 4$
29
29

In going to the half-capacity size units, the following two generalizations can be made:

(1) The half-capacity units san be run at the next higher synchronous motor speed without increasing the required NPSH level.

(2) The half-capacity designs are comparable to the fullcapacity designs in that the specific speed numbers are approximately the same. The half-capacity units can be modeled down from the full capacity units. The model ratio $=$ approximately 0.74 . 


\section{E.M. \#3131}

\section{G. SPECIAL HYDRAULICS PROBLEM AREAS INVOLVED IN THE DESIGN AND DEVELOPMENT}

OF A PUMP FOR HIGH TEMPERATURE LIQUID METAIS

1. The liquid metal plant system demands a pump whlch requires a low net positive suction head, and this is a primary consideration in the design of the pump hydraulics. The low NPSH requirement will Involve close attention to the design of the hydraulics approach channels, impeller inlet areas, and the impeller blade inlet design. The fluid analytical techniques used to solve this problem in a water pump design will be applicable to liquid metals, and the resulting low NPSH design should be very similar to that for a water pump. To determine the degree of conservatism to be used in designing liguid metal hydraulics will require an understanding of the erosion and cavitation effects of liquid metals as compared to water. As a part of the design, it will be mandatory to search out any available information on this.

One design area which could be a problem of a special nature, is the extra large diameter shaft associated with the liquid metal pump. Because of the very high temperature liquid metal, extra shaft diameter is required to transmit power to the impeller. In the case of the double suction primary pump design, this extra shaft diameter could interfere with the impeller inlet design. A more detailed study of this situation could possibly show that the effectiveness of a double suction impeller for low NPSH might be seriously limited for liquid metal pumps.

2. Due to the large size of these units, it is expected that the hydraulic performance will be verified by special model testing. The pump model laws are well established, and pump industrial experience has proven that model testing is an acceptable and accurate means for verifying head-flow, hydraulic efficiency, and hydraulic thrust performance.

3. One of the critical areas in the design of these liquid metal pumps is involved in the design and development of the hydrostatic radial bearing. Therefore, an important consideration in the design of the pump hydraulics will be the hydraulic radial thrust imposed by the pump. This will involve special design of the pump guide vanes and volutes to arrive at a proper design to obtain an acceptable radial thrust component. Hydraulic model tests should be designed to obtain radial thrust test data to confirm the hydraulic radial thrust analytical computations. 


\section{E.M. \#3231}

4. Bupply pressure to the liquid metal hydrostat1c radial bearing w1ll involve careful consideration. The obvious supply source is from the discharge of the main impeller. However, this source will be subject to changing pressure with a variation in flow. Further studies might indicate that a separate hydrostatic bearing fluid supply source w111 be required. Again, analytical techniques should be sufficient to determine the approach to this solution.

5. The liquid metal level control within the vertical pump colum is possibly a hydraulic problem. A solution here is probably tied into the system design and operation, and probably should be deferred until a better understanding of the plant system design has evolved. 


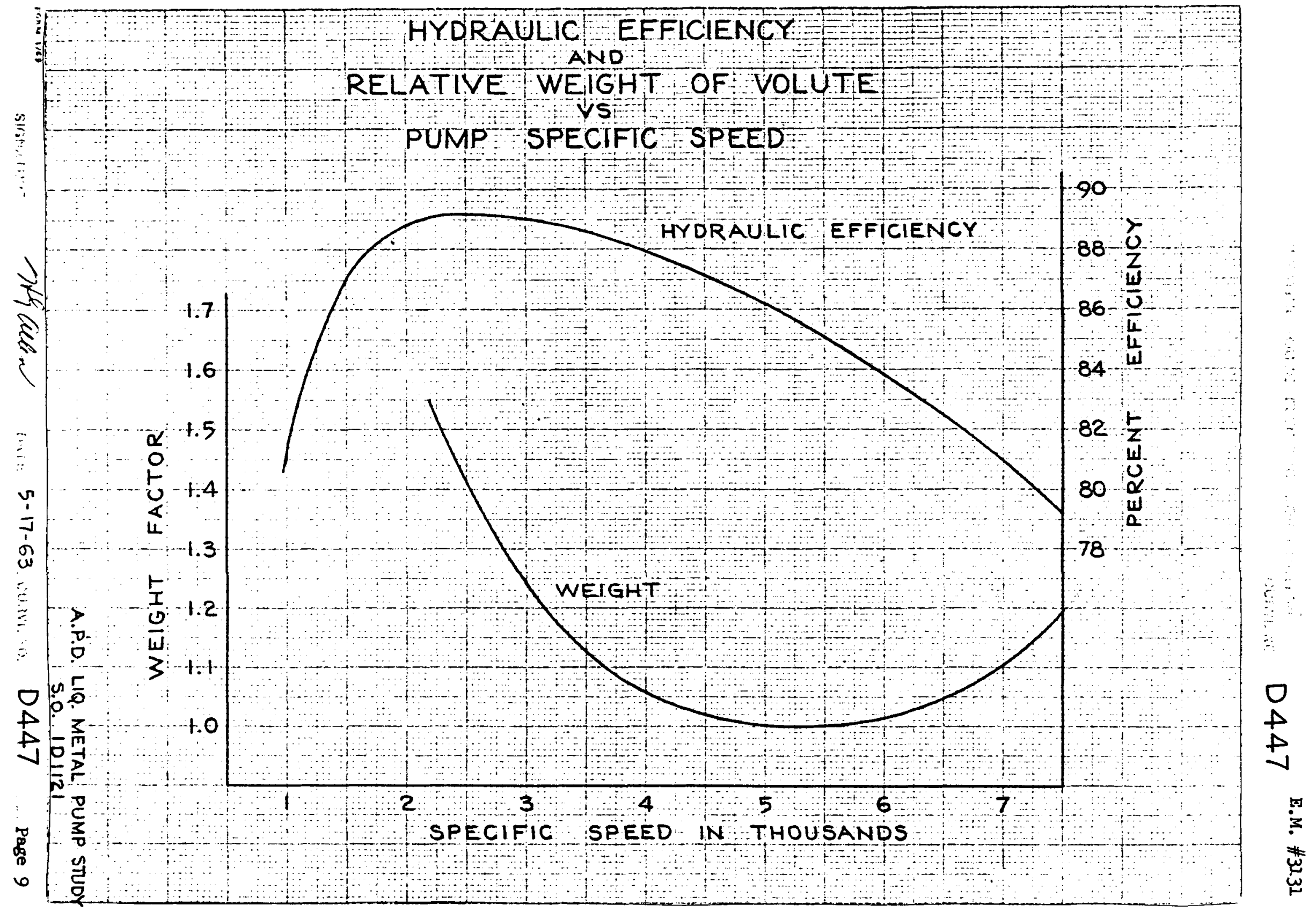


cuiven 0448

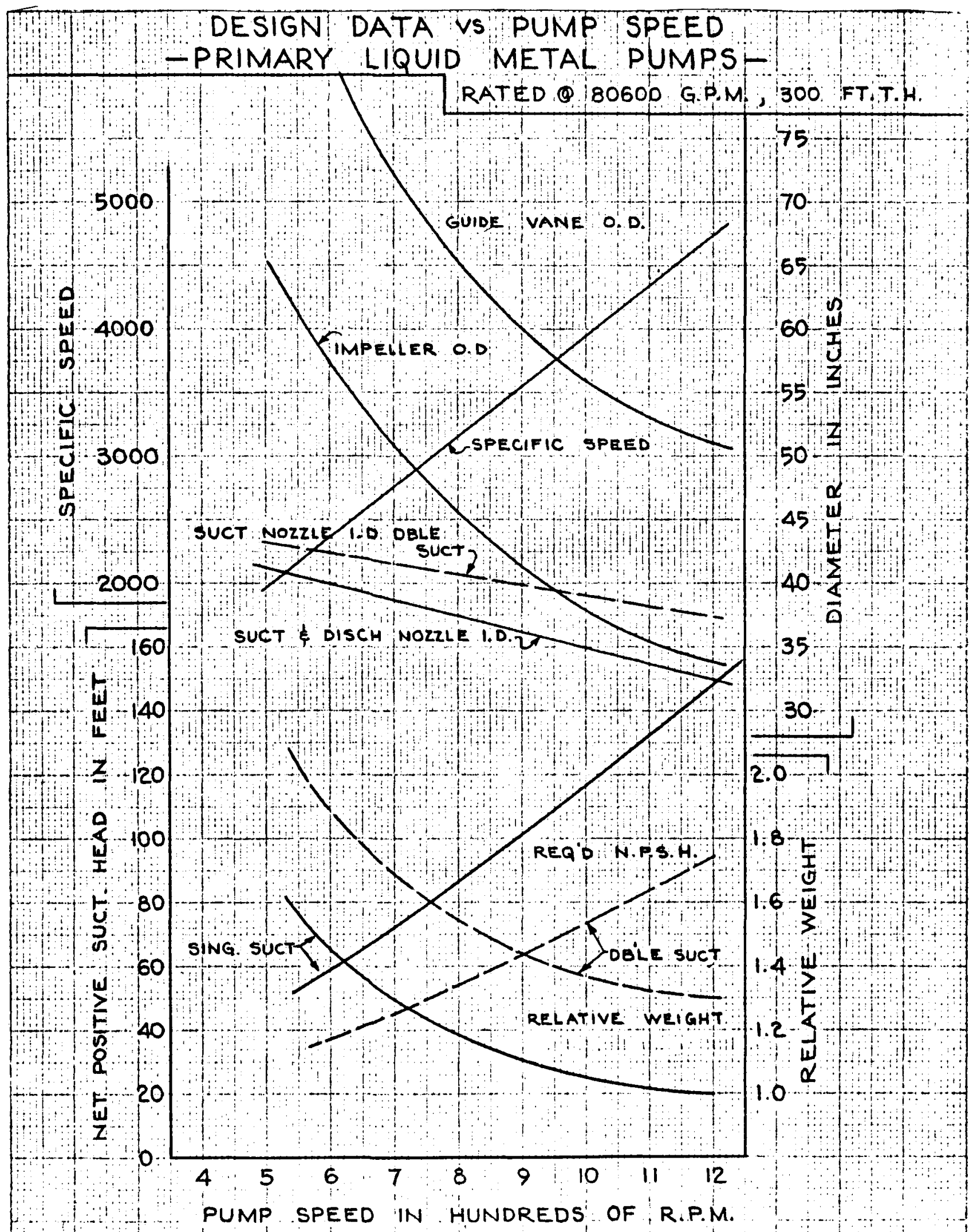

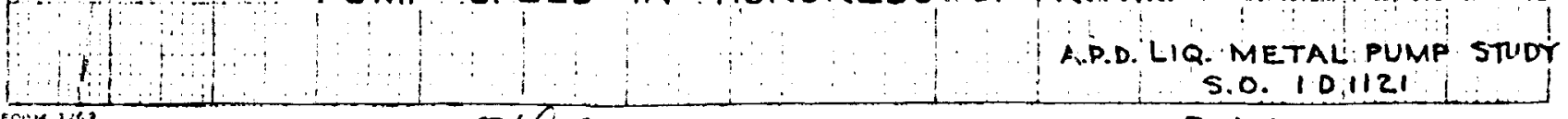

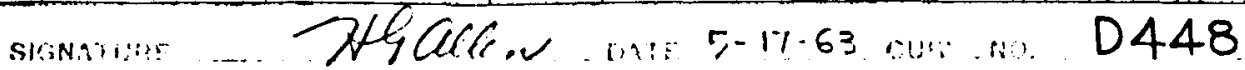




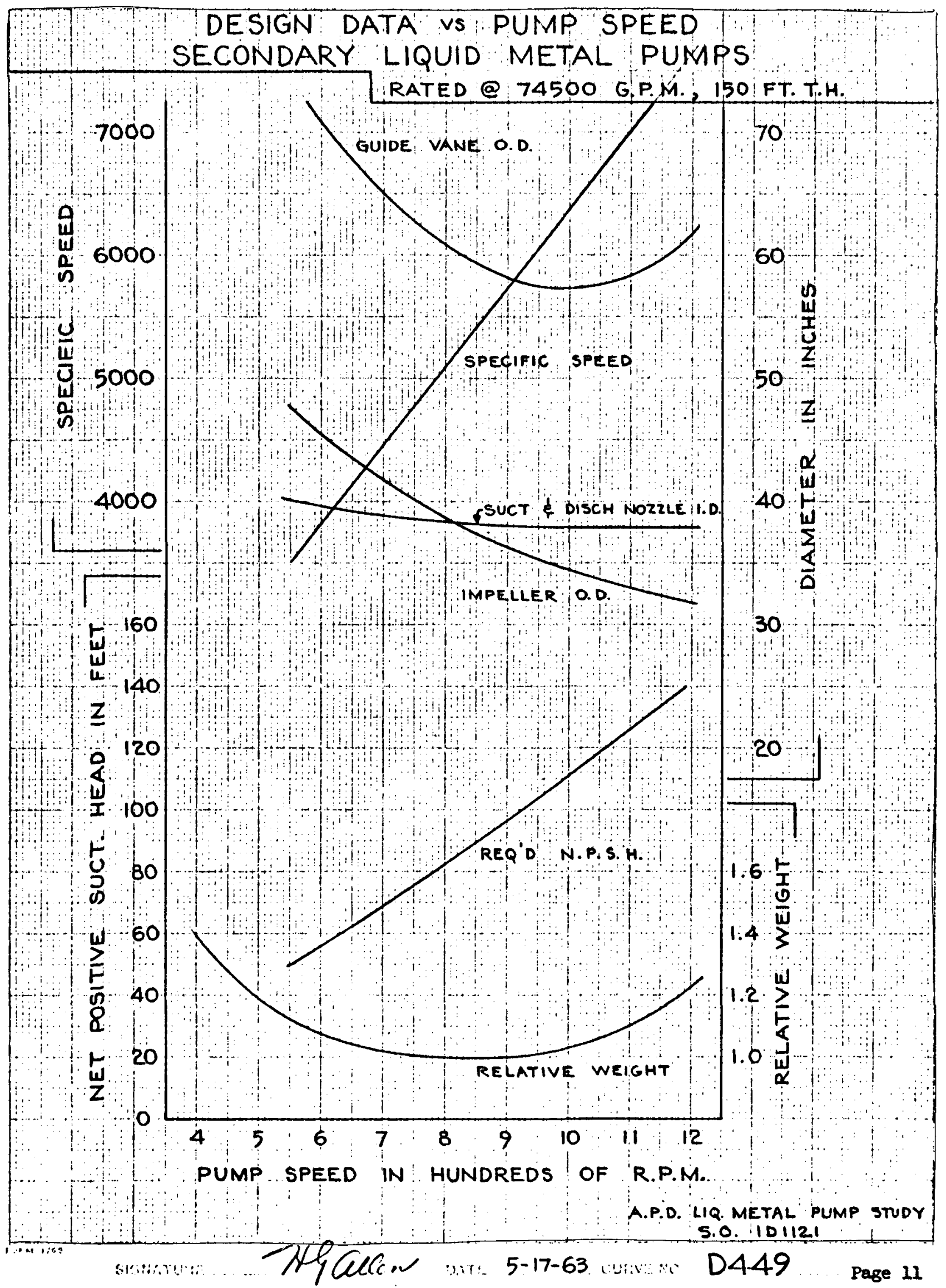




\section{APPENDIX 2 WAED EM 3123}

\section{SODIUM PUMP DESIGN STUDY}

SHOP ORDER IDII2I

DRIVE MOTOR STUDY

by

J. G. Wilhite

May, 1963

E. M. No. 3123

WESTINGHOUSE ELECTRIC CORPORATION

ATOMIC EQUIPMENT DIVISION

Cheswick, Pennsylvania 
1. General Requirements of Drive Motor

1.2 Power Requirements

1.3 Shaft Seals vs Canned Motors

1.4 Speed Requirements

1.5 Voltage Requirements

2. Wound Rotor Induction Motor
2.1 Advantages
2.2 Disadvantages
2.3 Motor Specifications
2.4 Hand Calculations
2.5 Development Required

3. Canned Single Speed Induction Motor

3.1 Advantages

3.2 Disadvantages

3.3 Motor Specifications

3.4 Computer Calculations

3.5 Variable Frequency Power Supply

3.6 Electromagnetic Coupling for Alternator Drive

3.7 Coupling Controller

3.8 Development Required

4. Conventional Single Speed Induction Motors with Magnetic Couplings

4.1 Advantages

4.2 Disadvantages

4.3 Motor Specifications

4.4 Magnetic Coupling

4.5 Coupling Controller

4.6 Development Required

5. Two Speed Canned Motors

5.1 Advantages

5.2 Disadvantages

5.3 Motor Specifications

5.4 Computer Calculations

5.5 Development Required 
TABLE OF CONTERTS (continued)

Page

6. Two Speed Conventional Motors

6.1 Advantages

6.2 Disadvantages

6.3 Motor Specifications

6.4 Computer Calculations

6.5 Development Required

7. Electromagnetic Pumps

7.1 Advantages

7.2 Disadvantages

7.3 Development Required

7.4 Reference Data 
E.M. \# 3123

SODIUM PUMP DESIGN STUDY

I. General Requirements of Drive Motor

1.2 The power required to drive the primary and secondary sodium loop pumps was determined from the head and flow given in SPD201-1 by Mr. C. C. RandaIl on 1-14-63. The primary pump requires 5500 horsepower and the secondary pump requires 2800 horsepower.

1.3 Shaft seals are permissible and have been considered in the explorative study of various types of driving motors. The evaluation of shaft seals and bearings is given in the attached report by $\mathrm{Mr}$. John Boyd and Mr. A. A. Ralmondi of Westinghouse Research Laboratories.

1.4 The shaft speeds were determined from the hydraulic design study reported by Mr. H. Allen. The primary pump speed is $900 \mathrm{rpm}$ and the secondary pump speed is $720 \mathrm{rpm}$.

1.5 Each plant is to have three primary loops and three secondary loops. The voltage selection was based on previous experience in building and designing pump motors at 440 volts, 2300 volts and 4000 volts, and a previous feasibility study made on 6900 volt motors. The 440 volt motor designs are limited by the turns per coll to about 1000 horsepower in size. This is far below the horsepowers required for elther the primary or the secondary pump. A 2300 volt design is quite practical, and can be used as conveniently as the 4400 volt design. However, many of the present power plant auxiliary equipment systems are at 4000 volts. The 4000 volt motor designs have additional ground insulation over that for the 2300 volt designs. Several commercial pump designs at 4000 volts have been built. The experience has proven the adequacy of the insulation system. previous study investigated the feasability of using 6900 volts, the next higher standard voltage, for canned motor designs. At that time, it was shown that the extra insulation, creepage distance and lower efficiencies made this higher voltage design undesirable. However, it would not be as undesirable on conventional uncanned motors and might be reconsidered if there appears to be a plant system preference for 6900 volts. Therefore, the motors investigated by this study and reported herein are all 4000 volt motor designs.

\section{Wound Rotor Induction Motor Drive}

Wound rotor induction motors with shaft seals and water rheostats in the rotor circuits for speed control are the preferred drive systems for the following reasons: 
E.M. \#3123

\subsection{Advantages:}

2.1.1 A single full capacity machine is required for each pump to deliver the required horsepower over a 4 to 1 speed range. (The speed range specified is 3 to 1 . Therefore, there is some speed control margin if it is desirable).

2.1.2 The operating efficiency is quite high. Above $90 \%$ tor loads of $50 \%$ of full load and above.

2.1.3 Speed control is smooth and stepless. It can be obtained either by controlling the level of the electrolyte in the water rheostat or by controlling the depth to which the electrodes are submerged into the electrolyte.

2.1.4 The motor is separate from the loop piping and is easily accessible for inspection and maintenance.

2.1.5 Inspection and maintenance are simple and conventional, and do not require special procedures or skills.

2.1.6 The water rheostat absorbs the high rotor losses during starting at slow speeds which eliminates the necessity of providing for cooling the rotor during such operations.

2.2 A few minor disadvantages are inherent in the wound rotor induction motor drive system. These are:

2.2.1 Conventional bearing lubrication and periodic maintenance of the bearings, and lubricating system are required. This is minor, because the motor is in the open and access to the bearings is easy.

2.2.2 The wound rotor motor has slip rings and carbon brushes which are not entirely maintenance-free. The carbon brushes need to be replaced and the slip rings may require resurfacing. The pump must be stopped for these maintenance operations. This means that at least one of the loops must be shut down for short periods of time, and cannot be considered to be maintenance-free.

2.2.3 Shaft seals would be required for this system. The water rheostat requires some maintenance which normally can be done while power is on the motor. If control is obtained by mechanically raising and lowering the electrodes, some types of maintenance on the mechanical mechanisms will require that the pump be de-energized. If water level con- 
trol is used, much of this type of maintenance is eliminated. The water level control system likely will require some maintenance.

\subsection{Wound rotor motor specifications}

2.3.1 The wound rotor induction motor for $5500 \mathrm{HP}, 900 \mathrm{rpm}$ would be a frame size 8 - 62-1/2-25 4000 volt, 3 phase, 60 cycle, $78^{\prime \prime}$ outside diameter, $95^{\prime \prime}$ height (length) 27,000 lbs. total weight with the following calculated performance at full speed:

$\begin{array}{llll}\text { Load } \% & 100 & 75 & 50 \\ \text { Efficiency \% } & 95.5 & 95.3 & 94.5 \\ \text { Power Factor \% } & 90 & 88.5 & 84\end{array}$

Secondary volts $=2000$, secondary amperes at full load $=1300$.

2.3.2 The wound rotor induction motor for $2800 \mathrm{HP}, 720 \mathrm{rpm}$ would be a frame size 10-62-1/2-20 4000 volt, 3 phase, 60 cycle, $78^{\prime \prime}$ outside diameter, $85^{\prime \prime}$ height (length) 21,000 lbs. total motor weight with the following calculated performance at full scale"

$\begin{array}{llll}\text { Load } \% & 100 & 75 & 50 \\ \text { Efficiency } \% & 94.5 & 94.5 & 93.5 \\ \text { Power Factor } \% & 90 & 88 & 83\end{array}$

2.4 Calculations for the wound rotor designs were done by hand by $\mathrm{Mr}$. E. F. Merrill of Westinghouse LRAD, 5NS, East Pittsburgh. Computer programs are not avaizable for special wound rotor designs. The method of performance calculations used is the standard equivalent circuit method.

2.5 The production of these wound rotor motors would not require any development in any area which might be considered to be an unknown area. The use of such conventional motors is dependent on the development of the shaft seals.

3. Canned, Single Speed Induction Motors

The second in preference is a single speed canned motor drive with any of several types of variablë frequency power supply.

\subsection{Advantages:}

3.1.1 The canned motor is seal welded into the pump loop system and is, therefore, a zero leakage type of pump drive. 
3.1.2 The drive motor rotor and its thrust and radial bearings are inside the pump loop. The bearings are self lubricating, long life and essentially maintenance free.

3.1.3 Periodic inspection and maintenance are not required.

3.1.4 The motor windings are inside a sealed shell and are, therefore, not subject to insulation deterioration from oxidation or contamination from air-born dirt and molsture.

3.1.5 Because the canned motor is maintenance-free, it can be on the line 100\% of the time.

\subsection{Disadvantages:}

3.2.1 One major disadvantage is that a variable frequency power supply is required. This means that full power capacity must be provided in three machines and possibly four machines in addition to that of the motor. This makes a total of four or five full capacity machines for each pump.

3.2.2 The efficiency of the overall power supply and motor drive system is considerably less than for the wound rotor motor drive system. First, the canned motor efficiency is somewhat lower than that of the wound rotor motor. It is between 67 and $78 \%$ at loads of $50 \%$ of full load and above. In addition, the power supply efficiency is in the order of 85 to $90 \%$. Therefore, the system efficiency is in the order of 57 to $70 \%$.

3.2.3 The power supply is not considered to be maintenance-free. The synchronous motors and alternators would have slip rings and carbon brushes which require periodic maintenance. If speed control is obtained by d-c to a-c motor generator sets, even more brush and commutator maintenance will be required.

\subsection{Canned, Single and Speed Motor Specifications}

3.3.1 The single speed canned induction motor for $5550 \mathrm{Hp}, 900 \mathrm{rpm}$ would be a Series 10,045, Type 8P24-110, 4000 volt, 3 phase, 60 cycle, $37^{\prime \prime}$ outside diameter of core, 200" height (length) with the following calculated performance:

$\begin{array}{lcll}\text { Load } \% & 100 & 75 & 50 \\ \text { Efficiency \% } & 78 & 76 & 67 \\ \text { Power Factor \% } & 69.5 & 67.4 & 58.1\end{array}$


3.3.2 The single speed canned induction motor for $2800 \mathrm{Hp}, 720$ rpm would be a Serles 9019, Type 10P20-100 4000 volt, 3 phase, 60 cycle, 29" outside diameter of core, 185" height (length) with the following calculated performance:

$\begin{array}{lrll}\text { Load } \% & 100 & 75 & 50 \\ \text { Efficiency } \% & 85 & 82 & 79.6 \\ \text { Power Factor\% } & 73.1 & 68.1 & 62.1\end{array}$

3.4 Canned motor computer calculations

The performance calculations for the primary and secondary canned motors were made on the IBM 7094 computer in East Pittsburgh using a performance program specially written by WAED engineers for canned motors with fluid in the magnetic gap. This program is based on the standard equivalent circuit method for performance calculations with specific sections for items peculiar to these motors such as can loss, fluid friction loss, winding temperature, starting requirements and search subroutines for calculating the performance of any pre-selected horsepower or temperature rise.

\subsection{Varlable frequency power supply}

Three types of variable frequency power supply systems were considered in this study and a description and evaluation of each type of system follows:

3.5.1 The preferred type of variable frequency power supply consists of a synchronous motor driving, a synchronous generator through a magnetic type of shaft coupling. This is preferred because only three full capacity machines are required to provide full capacity for each pump over a 3 to 1 speed range. The magnetic coupling would require excitation power but this is a small percentage of the total power involved. Speed, and thus frequency control, would be obtained by controlling the excitation to the coupling. The capacity for each of the three machines would be in the same order of magnitude as the pump motor capacity. Line starting would not be provided because this would require power supply capacities in the order of four or five times the pump motor capacity. Instead, the pump motor would be started with the power supply M-G set at zero speed. The pump motor would be connected electrically to the $M-G$ set, and then the $M-G$ set would be started on the line. The pump and M-G set would thus be started up together from zero speed. Only the full load pump capacity for the rating of each machine plus losses would be required with this type of starting. 
3.5.2 A second type of variable frequency power supply would be the Ward-Leonard type which includes two M-G sets. One set would have a synchronous motor driving directly a-c generator. The second $M-G$ set would have a d-c variable speed motor driving directly an a-c generator. The d-c motor would be electrically connected to the d-c generator and speed control would be obtained by voltage control on the d-c generator. The pump motor would be electrically connected to the a-c generator on the second or variable speed $M-G$ set. The frequency output would be directly proportional to speed of the second $M-G$ set.

3.5.3 A third type of variable frequency power supply is the Ignition cyclo-converter system which employs Ignition rectifiers to convert $a-c$ power to $d-c$ power and then to convert the $d-c$ power to a variable frequency a-c. The present state of development of this system is not considered to be adequate for supplying large blocks of inductive loads. Furthermore, the low power factor of induction motors during starting and higher power factor at full speed makes power factor correction a continiously varying factor. Arc-backs and miss-fires are quite frequent in the systems presently operating. These spurlous faults would render the system unsuitable for power plant operation where continuous operation is important.

\subsection{Electromagnetic coupling for alternator drive}

The electromagnetic couplings for the M-G sets which would supply variable frequency to the primary pump motors and the secondary pump motors would have sufficient capacity to supply the rated horsepower. of the individual pump motors plus the losses in each alternator.

\subsubsection{The electromagnetic coupling for the primary pump M-G set would have an approximately rating of 6900 horsepower at $1200 \mathrm{rpm}$ with a speed range of 3 to 1 .
3.6.2 The electromagnetic coupling for the secondary pump M-G set would have an approximate rating of 3600 horsepower at $1200 \mathrm{rpm}$ with a speed range of 3 to 1 .

3.7 The Coupling Controllers would be electronic exciter units mounted in NEMA type I ventilated enclosures. The controller capacity for the primary pump $M-G$ set coupling would be approximately $25 \mathrm{KW}$. The capacity for the secondary pump $M-G$ set coupling would be approximately $10 \mathrm{KW}$. 
E.M. \#3123

\subsection{Development required.}

Development and work beyond the scope of this particular study would include the investigation of $M-G$ sets with capacities to drive three pumps each. With present knowledge, it appears that the couplings for such large frequency changer sets would be larger than those presently in production. If individual $M-G$ sets are used for each pump, the couplings would require only a small amount of development work to assure proper application of present production couplings and controllers.

A normal amount of development work would be needed for the canned motors which are larger than those presently in production. Proven systems, materials and designs would be used which would require only a small amount of development because of the large size motors.

\section{Conventional Induction Motors with Magnetic Couplings}

A third type of drive for the primary and secondary pumps employs squirrel cage induction motors and conventional design with electromagnetic couplings between each motor and pump.

\subsection{Advantages}

4.1.1 Two full capacity machines are required for each pump to deliver the required horsepower over a 3 to 1 speed range.

4.1.2 The operating efficiency is quite high, approximately 85 to $90 \%$ for loads of $50 \%$ of full load and above.

4.1.3 Motors of conventional designs would have lower initial cost than canned motors of the same capacity.

4.1.4 Speed control is smooth and stepless. It would be accomplished by controlling the excitation to the electromagnetic couplings.

4.1.5 The motor is separate from the loop piping and is easily reached for inspection and maintenance.

4.1.6 No carbon brushes or slip rings would be used in the drive motors. 


\subsection{Disadvantages}

4.2.1 Shaft seals would be required for this system.

4.2.2 Conventional bearing lubrication and periodic maintenance of the bearings and lubrication system are required.

4.2.3 Periodic shutdown of the pumps would be required for inspection and maintenance of bearings.

\subsection{Conventional Motor Specifications}

4.3.1 The squirrel cage induction motor for $5500 \mathrm{HP}, 900 \mathrm{rpm}$ would be a frame size 8 - 62-1/2 - 25, 4000 volt, 3 phase 60 cycle, 78 " outside diameter, 85 " height (length) 25,000 Ibs. total weight with the following estimated performance at full speed.

$\begin{array}{lccl}\text { Load \% } & 100 & 75 & 50 \\ \text { Efficiency \% } & 95.5 & 95 & 94.5 \\ \text { Power Factor \% } & 91 & 89 & 85\end{array}$

4.3.2 The squirrel cage induction motor for 2800 horsepower, $720 \mathrm{rpm}$ would be a frame size $10-62-1 / 2-20$, 4000 volt, 3 phase, 60 cycle, 78 " outside diameter, $80 "$ height (length) 20,000 lbs. total motor weight with the following estimated performance at full speed:

$\begin{array}{lccl}\text { Load \% } & 100 & 75 & 50 \\ \text { Efficiency \% } & 94.5 & 94 & 93.5 \\ \text { Power factor \% } & 91 & 89 & 84\end{array}$

4.4 The electromagnetic couplings would be similar to the following Dynamatic couplings manufactured by the Dynamatic Division of the Eaton Manufacturing Company, Kenosha, Wisconsin:

4.4.1 The $5500 \mathrm{HP}, 900 \mathrm{rpm}$ coupling would be similar to the Dynamatic Model 5TWC26 adjustable speed, rotating field, water-cooled, eddy-current coupling with the following torque-speed characteristics:

$32800 \mathrm{lb}$. ft. at $23 \mathrm{rpm}$ slip

$41900 \mathrm{lb}$. ft. at $30 \mathrm{rpm}$ slip

$49300 \mathrm{lb}$. ft. at $40 \mathrm{rpm}$ slip

$52700 \mathrm{lb}$. ft. at $50 \mathrm{rpm}$ slip

$66600 \mathrm{lb}$. ft. at maximum slip

Coupling is capable of dissipating $1100 \mathrm{HP}$ at $900 \mathrm{rpm}$ input speed. Speed range is 3 to 1 . 
4.5 The controllers for the electromatic couplings would be similar to the following Dynamatic electronic excitation and speed control units mounted in NEMA Type 1 ventilated enclosures.

4.5.1 The controller for the $5500 \mathrm{HP}$ primary pump coupling would be similar to the Dynamatic Model B-200 which has a rating of $20 \mathrm{KW}$.

4.5.2 The controller for the $2800 \mathrm{HP}$ secondary pump coupling would be similar to the Dynamatic Model B-75, which has a rating of $7.5 \mathrm{KW}$.

4.6 Development Required

The application of this type of drive system is dependent upon the development of shaft seals. Further development would be only that required for the combining of the conventional motor, coupling and pump into a single drive system. Each component is presently in production and would require no special development.

\section{Two Speed Canned Motors}

A fourth type of drive system using two speed canned motors would be practical if stepped flow is acceptable. With three loops in the primary system and secondary system, the following flow control would be obtained:

Pump No.

of Flow

$100 \%$

83.3

$50 \%$

$50 \%$

$33 \%$

$33 \%$

$16.7 \%$

0
1

$100 \%$
50
0
0
50
0
0
0
0

Per Cent Speed

\subsection{Advantages}

$\begin{array}{cc}100 \% & 100 \% \\ 100 & 100 \\ 100 & 100 \\ 50 & 100 \\ 50 & 50 \\ 50 & 50 \\ 0 & 100 \\ 0 & 50 \\ 0 & 0\end{array}$

5.1.1 A single full capacity machine would be used to supply full pumping capacity over a possible flow range of 6 to 1 (in steps). 
5.1.2 The system would be seal welded and have zero leakage.

5.1.3 No $M-G$ sets or frequency changer equipment would be used.

5.1.4 The drive motor rotor and its thrust and radial bearings are inside the pump loop. The bearings are selflubricating, long life and essentially maintenance free.

5.1.5 Periodic inspection and maintenance are not required.

5.1.6 The motor windings are inside a sealed shell and are therefore not subject to insulation deterioration from oxidation or contamination from alr-born dirt and molsture.

5.1.7 Because the canned motor is maintenance free, it can be on the line $100 \%$ of the time.

\subsection{Disadvantages}

5.2.1 The major deviation from the E.Spec. Is the stepped flow method of operation as mentioned above.

5.2.2 The efficiency is somewhat lower than that of the wound rotor motor system described in section 1 .

\subsection{Two Speed Canned Motor Specifications}

5.3.1 The two speed canned induction motor for $5500 \mathrm{HP}, 900 \mathrm{rpm}$ would be a Series 10,045 Type 8/16P24-110, 4000 volt, 3 phase, 60 cycle $37^{\prime \prime}$ outside diameter of core, 200 " helght (length) with the following estimated high speed performance:

$\begin{array}{llll}\text { Load } \% & 100 & 75 & 50 \\ \text { Efficiency \% } & 78.3 & 73 & 65 \\ \text { Power Factor \% } & 70.7 & 65.5 & 57.5\end{array}$

5.3.2 The two speed canned induction motor for $2800 \mathrm{HP}, 720 \mathrm{rpm}$ would be a Series 9019, type 10/20 P20-100, two winding, 4000 volt, 3 phase 60 cycle, 30 " outside diameter of core, 185" height (length) with the following estimated high speed performance:

$\begin{array}{lrrr}\text { Load } \% & 100 & 75 & 50 \\ \text { Efficiency \% } & 84 & 81 & 78 \\ \text { Power Factor \% } & 71 & 66 & 60\end{array}$




\subsection{Canned Motor Computer Calculations}

The performance calculations for the primary two speed canned motor were made on the IBM $709 / 4$ program described in section 3.4. The complete computer print-out sheets for the primary pump are in Sec. 8. The design and performance for the secondary pump motor were estimated; computer calculations were not made.

\subsection{Development Required}

A normal amount of development work would be needed for the two speed canned motors which are larger than those. presently in production. Proven systems, materials and designs would be used which would require only a small amount of development because of the large size motors.

\section{Two Speed Conventional Motor Drive}

A fifth type of drive system using two speed conventional industrial Induction motors would be practical if stepped flow is acceptable. The flow control would be the same as that glven in section 5 .

\subsection{Advantages}

6.1.1 A single full capacity machine would be used to supply full pumping capacity over a possible flow range of 6 to 1 (in steps).

6.1.2 The operating efficiency is quite high, approximately 90 to $95 \%$ for loads of $50 \%$ of full load and above.

6.1.3 Motors of conventional designs would have lower initial cost than canned motors of the same capacity.

6.1.4 The motors are separate from the loop piping and are easily reached for inspection and maintenance.

6.1.5 No carbon brushes, slip rings or magnetic slip type couplings would be required.

\subsection{Disadvantages}

6.2.1 The major deviation from the E.Spec. is the stepped flow method of operation as mentioned in section 6 .

6.2.2 Shaft seals would be required for this system. 
6.2.3 Conventional bearings, lubrication and periodic maintenance of the bearings and lubrication system are required.

6.2.4 Periodic shutdown of the pumps would be required for inspection and maintenance of bearings.

6.3 Two speed conventional motor specifications

6.3.1 The conventional two speed squirrel cage induction motor for $5500 \mathrm{HP}, 900 / 450 \mathrm{rpm}$ would be a frame size 8/16-62-1/2-30 4000 volt, 3 phase, 60 cycle, $96 "$ outside diameter, 110 " height (length) 35,000 Ibs. total welght with the following calculated performance at full speed:

$\begin{array}{llll}\text { Load } \% & 100 & 75 & 50 \\ \text { Efficiency \% } & 95.5 & 95.7 & 95.2 \\ \text { Power Factor\% } & 93 & 94 & 93.2\end{array}$

This motor would have a water cooled stator.

6.3.2 The conventional two speed squirrel cage induction motor for $2800 \mathrm{HP} 720 / 360 \mathrm{rpm}$ would be a frame size $10 / 20-62-1 / 2-224000$ volt, 3 phase, 60 cycle, $96 "$ outside diameter, $97^{\prime \prime}$ height (length) 25,500 lbs. total weight with the following calculated performance at full speed.

$\begin{array}{lrll}\text { Load \% } & 100 & 75 & 50 \\ \text { Efficiency \% } & 95.3 & 95.3 & 94.8 \\ \text { Power Factor \% } & 92.2 & 91.6 & 88.2\end{array}$

This motor would have a water cooled stator.

6.4 Computer Calculations

The performance calculations for the primary and secondary canned motors were made on the IBM 7094 computer in East Pittsburgh using an LRAD design program.

6.5 Development Required

Ittle or no development work would be required beyond the shaft seal development being done as a part of this study. 
7. Electromagnetic Pumps

Electromagnetic pumps of many different flow patterns have been made for pumping liquid metals such as sodium. For the head and flow values of this design study, the rotating d-c magnet type with a spiral duct of several turns appears to be preferable. Efficiencies of such pumps with smaller capacities are less than $30 \%$. With head and flow values several times larger than those for pumps presently built, the efficiencies are likely to be lower. Furthermore, the area of cross-section of the flow must be several hundred square inches to allow a practical limit of velocity. This large area necessitates a magnetic gap of the order of 10 to 20 inches which appears quite impractical from the efficiency standpoint. A pump with several turns of the spiral flow pattern would be required to produce the high heads specified for this study. The hydraulic losses involved in a large number of turns in the spiral path tend to reduce the efficiency even further. Estimated efficiencies would be in the order of 10 to $20 \%$ for this size electromagnetic pump. It should be considered only for such applications as can sacrifice efficiency for some improvement in maintenance and in the elimination of rotating seals.

\subsection{Advantages}

7.1.1 The electromatic pump is $100 \%$ leak free.

7.1.2 No mechanical moving parts are involved in the pump.

7.1.3 Flow control is simple and continuous without steps.

\subsection{Disadvantages}

7.2.1 The major objection to electromagnetic pumps of this capacity is the extremely low efficiency estimated to be in the order of 10 to $20 \%$.

7.2.2 Excitation power and electrode current are quite large and require special generating equipment.

7.2.3 The pump dimensions would be quite large, probably larger than the largest motor-pump combination considered in this study.

\subsection{Development Required}

The development of the electromagnetic type of pump would involve a large amount of original work for a pump of this type. Primarily, it would be in the development of the optimum 
geometry for the magnetic circuits and flufd flow paths. Unknown parameters are the pumping efficiency and the head produced. The known parameters obtained from previous work on this type of pump are the electrical conductance of liquid metal, the fluid friction loss constants for liquid metal, and calculation methods.

\subsection{Reference Data}

7.4.I Electromagnetic Pumps, by R. Panholzer, Electrical Engineering, February, 1963.

7.4.2 Sodium Pump Technology by D. R. Nixon, Westinghouse Electric Corporation, WAED, EM No. 3082 and the references compiled therein. 
APPENDIX III

RECENT OPERATIONAL DIFFICULTTES

$\underline{O F}$

PRIMARY SODIUM PUMPS

IN

EBR II AND HNPF 


\title{
APPEIDIR III
}

\author{
RECENT OPERATIONAL DIFTICULTIES OF PRIMARY SODIUM
}

PUMPS IN EBR -II and HNPF

Operating difficulties have been encountered with the primary sodium pumps installed in EBR-II and HNPF. The history and nature of these difficulties as obtained from discussions with cognizant personnel connected with these reactors are reported in this appendix.

EBR -II-PUMPS

Pump Description

The FBR-II primary pumps are vertical single state centrifugal with totally enclosed motors. The pump nameplate rating is:

$\begin{array}{lll}5500 \text { GPM } & 54.5 \text { cycle } & 1075 \mathrm{Fs} \\ 700 \mathrm{~F} & 480 \mathrm{~V}, 3 \text { phase } & 200 \mathrm{TDH} \\ 350 \mathrm{HP} & & 0.875 \mathrm{sp} . \mathrm{gr} .\end{array}$

A variable speed drive is obtained by means of a constant speed motor which drives an alternator thru a variable slip DYNAMATIC coupling. The alternator supplies a variable frequency power to induction motor drives of the pumps. The pumps are inserted thru a plug in the top shield with the motors above the shield and the pump casing submerged in a pool of sodium. A photograph of the M-l pump at time of installation is shown in Figure 1 .

The pumps for the EBR-II were purchased on performance specifications after extensive testing of an experimental unit. This unit was rated at $5000 \mathrm{gpm}, 40 \mathrm{psi}$, at $700 \mathrm{~F}$ 。 It was operated at temperatures to $900 \mathrm{~F}$. The temperature of its grease lubricated motor bearings did not exceed $175^{\circ}$ 。 This pump was operated suceessfully for 16,000 hours at ANL with 250 starts. The operating time consisted of 7000 hours at full speed (1750 rpm), 3700 hours at half speet, and 5200 hours at $10 \%$ speed. Details of testing this unit for the first 7500 hours are reported in Reference 1.

A schematic cross section of the EBR-II pump is shown in Figure 2. This indicates the major design features of this type of pump. The lower bearing is a hydrostatic (fluid piston) liquid metal bearing. The journal contains four equally spaced pockets supplied with sodium from the pump discharge through a distributing orifice located in the center of each pocket. The free sodium surface in the pump case is maintained by the sodium level in the EBR-II primary tank. The inert gas is common in 

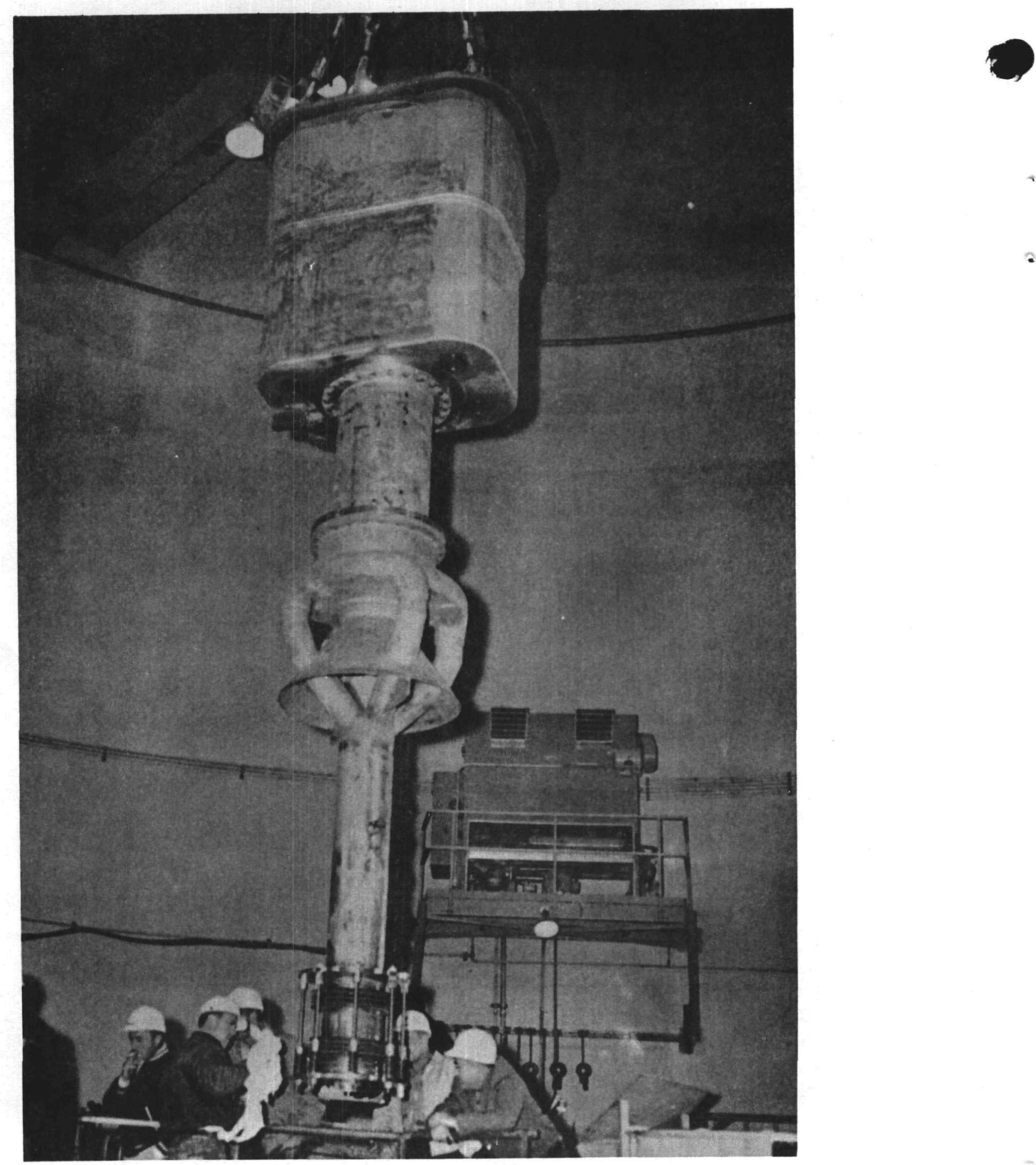


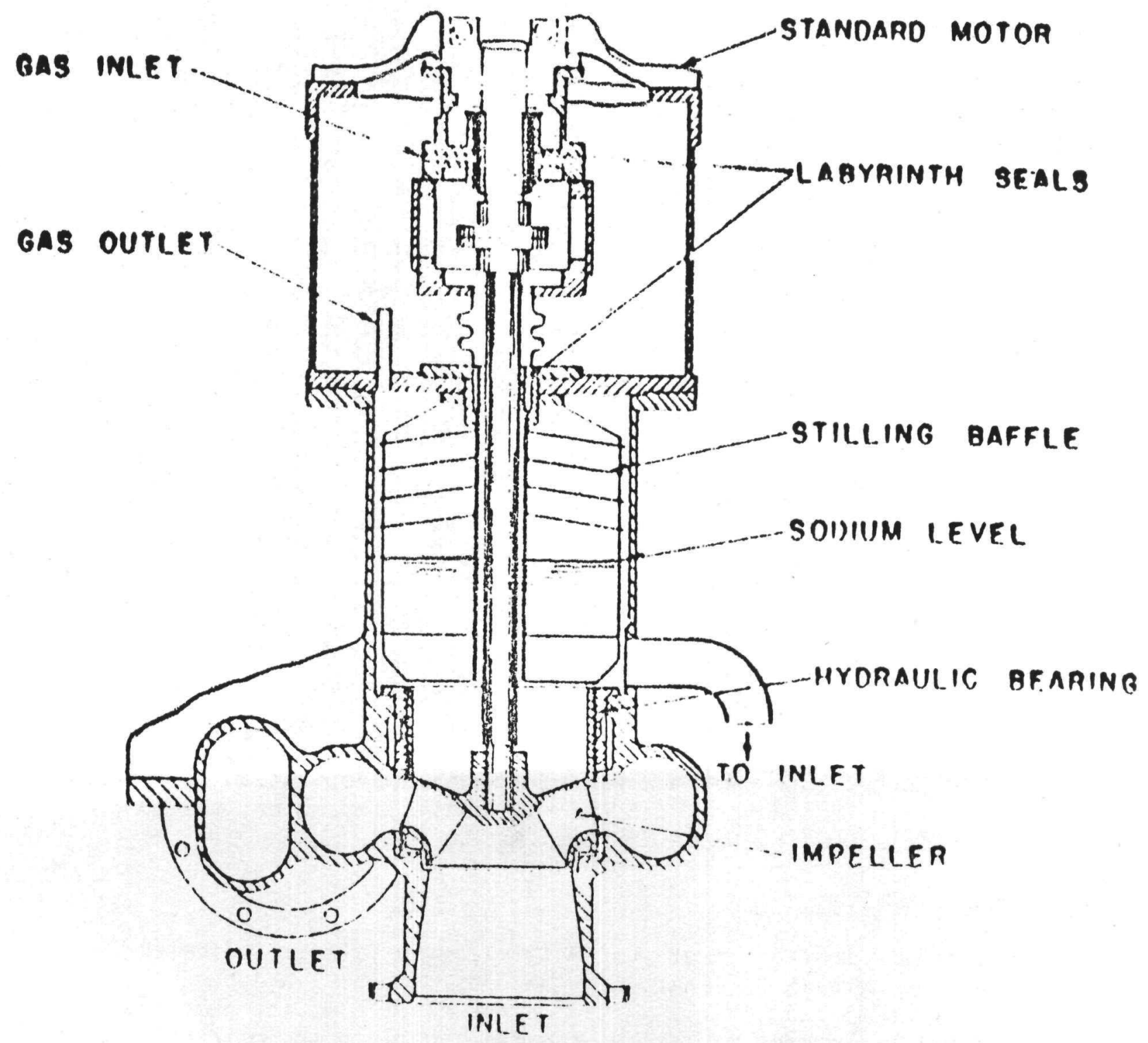

Figure 2. Infernal arrangement of mechanical pump 
the pump case and motor enciosure. Passage of sodium vapors into the motor enclosure is minimized by means of labyrinth seals on the pump shafts.

\section{Operating History}

The primary pumps $M-1$ and M-z were installed and operated during primary sodium flitration runs. The apronximsts flow rates and periods are as follows :

\section{Both Pumps}

April 16, 17, 1963

1 hour at $150 \mathrm{rpm}$ and $750 \mathrm{rpm}$

After one hour fump $M=1$ fsilied. $M-1$ was removed and replaced by a duminy plug.

\section{Purmo M-2}

June 1, 10, 1963

\begin{tabular}{ccc}
6 hours at $165 \mathrm{rpm}$ and $900 \mathrm{gpm}$ \\
16 & 310 & 1570 \\
18.6 & 510 & 2700 \\
12.6 & 660 & 3400 \\
63.0 & 760 & 4000 \\
38.0 & 760 & 4000 \\
\hline 154.6 & & \\
11 & & \\
20.5 & 440 & 2300 \\
0.7 & 600 & 3190 \\
& 645 & 3300
\end{tabular}

On August 18 th pump $M-$ faj $1 \in d$ after total operation of approximately 188 hours. Ijon rencval of the pump motor, the pump shaft showed $t h=$ same symptoms as for $M_{m} l$ 。

\section{Examination of $\mathrm{Y}_{\mathrm{ign}} \mathrm{M} \mathrm{M}$}

Disassembly and inspection of the clearance between the shinf and the jabyrinth seal had been lost and that the sect bsid severiy galifd on the shaft. If the pump shaft had origingliy sentered gerfecty yith the labyrinth the original radial clearance would have betr $0.0 \pm 5$ inches. Trotographs of the damaged shaft and seal are shown in Figs. $3,4,5$, and $\epsilon_{0}$

\section{Cause of Failure}

A contributing causs of the fallure misy rave been bowing or warping of the shaft as a result of inariquate stress relief heat treatment following welding of the three piecs fartinlly hollow shaft. Following welding the pump shafts were to have bern stress relieved at $900^{\circ} \mathrm{F}$ but were 


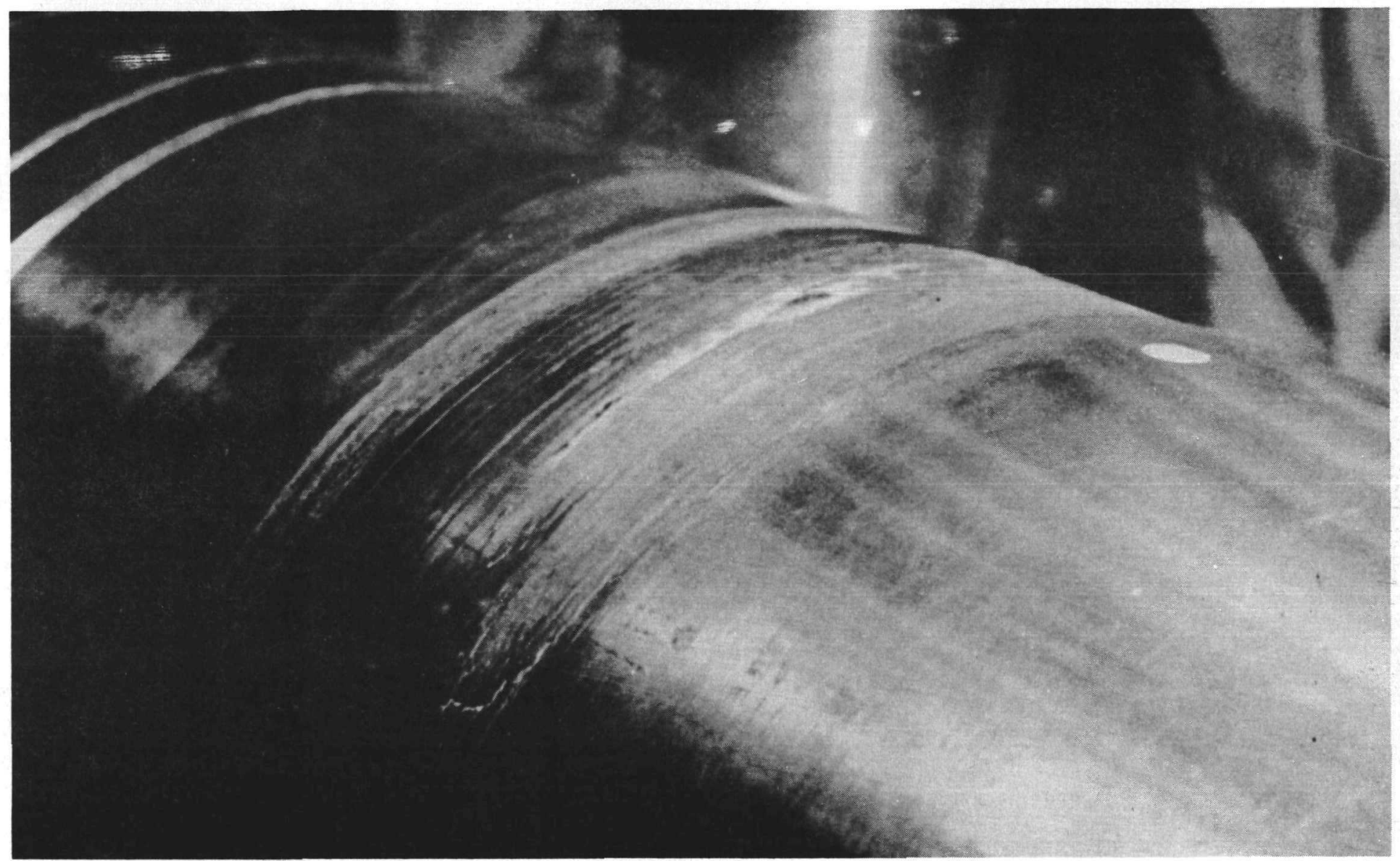

Fig. 3

Close-up of Gall Marks on M-1 Primary Pump Shaft 

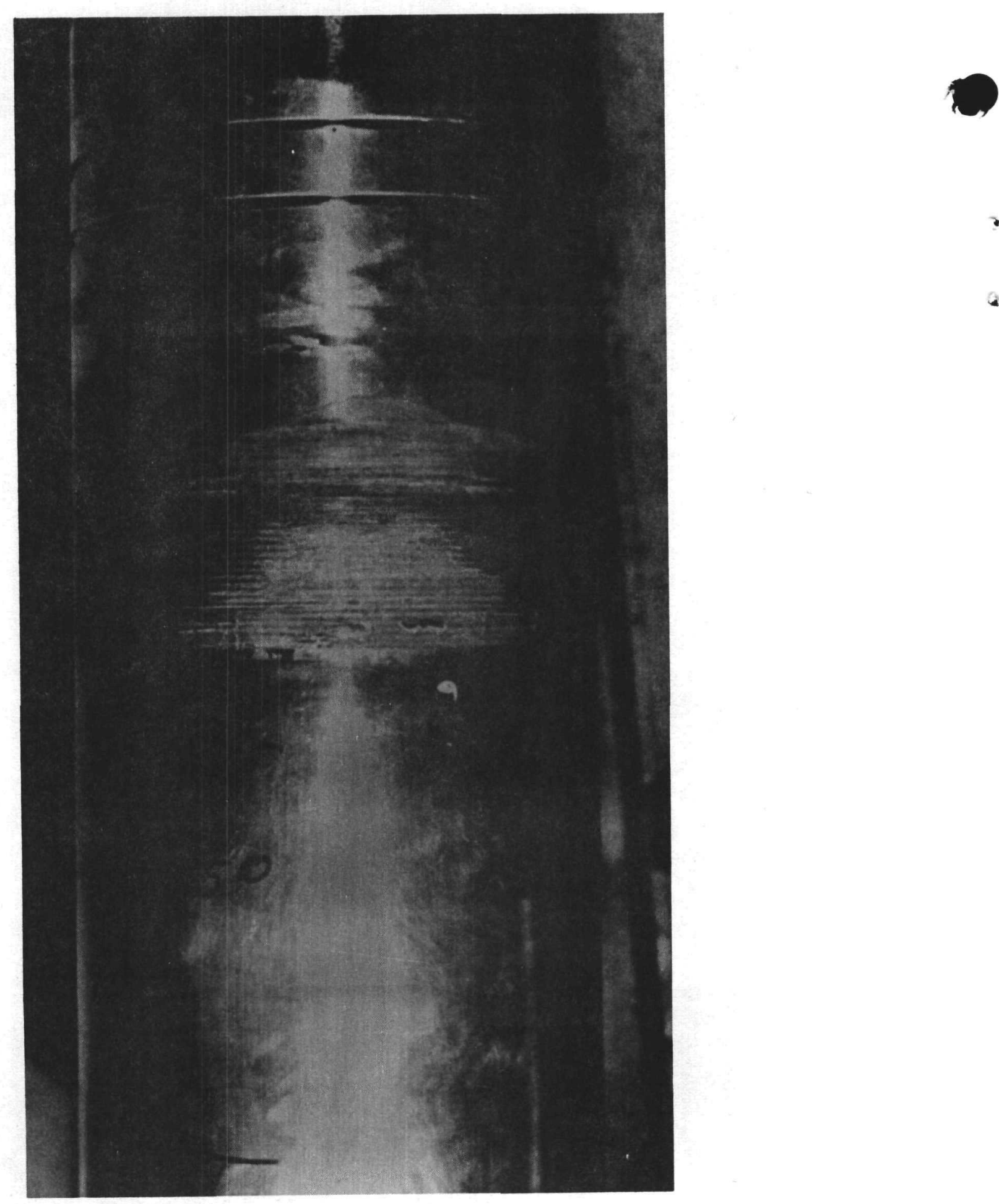

Fig. 4

Gall Marks on M-1 Pump Shaft

$-6-$ 


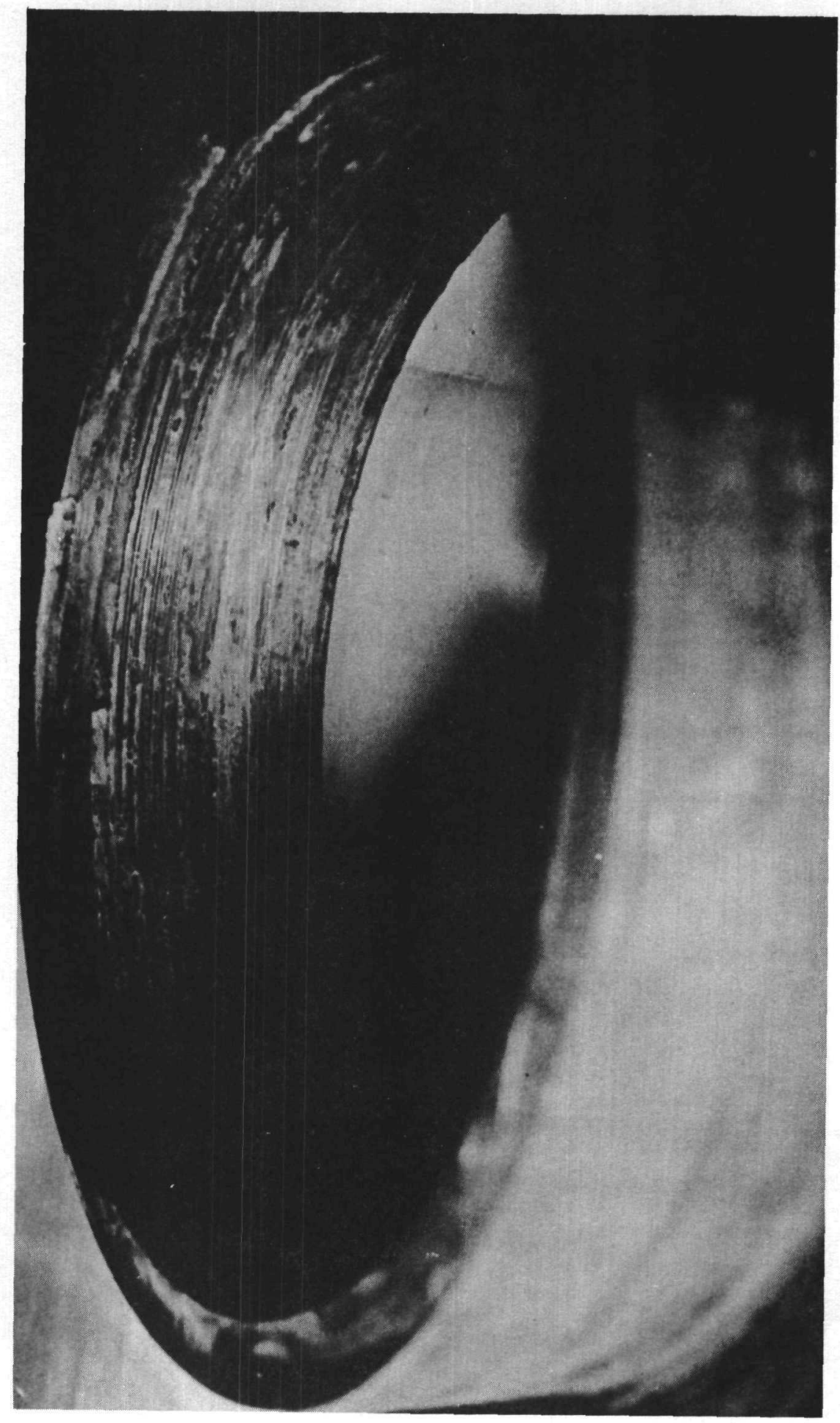

Fig. 5

Close-up of Damaged Labyrinth Seal of M-I Primary Pump 

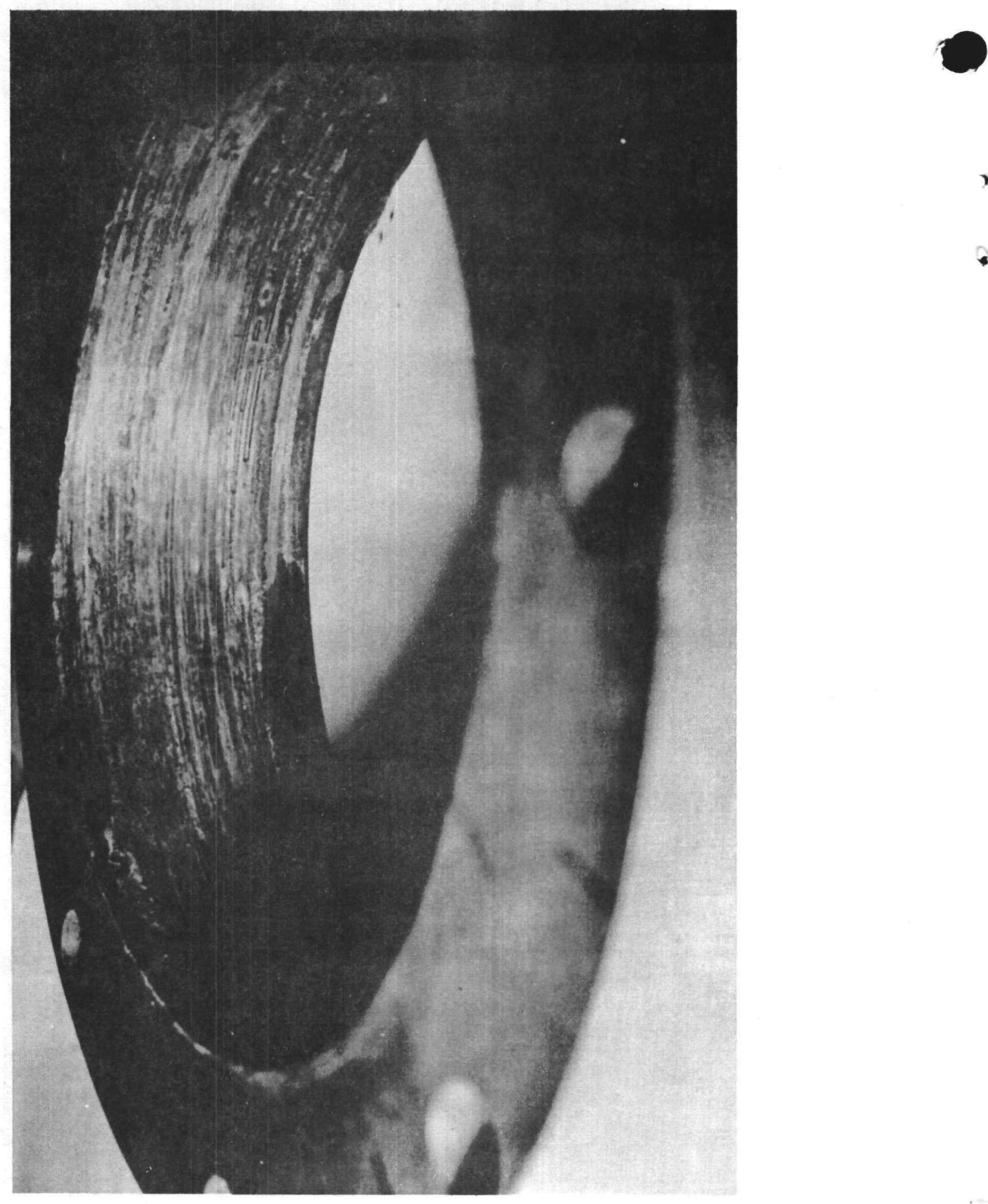
in fact only taken to $700^{\circ} \mathrm{F}$. Some structural deformations were found in the pump shield plug. The detail of labyrinth seal design by the pump builders of the prototype and the EBR-II reactor pumps is believed to have been more favorable in the prototype pump. In the prototype the labyrinth seal was fabricated with very thin ends on the leaves adjacent to the shaft and with relatively deep grooves between leaves. The reactor pump $\mathrm{M}_{\infty} \mathrm{I}$ was fabricated with much shallower grooves and thicker heavier leaves. In the prototype pump, shaft contact with the seal leaves due to any misalignment would cause rapid wearing away of the tips without causing serious damage to the shaft. In the later design the heavy (thick) tip ends and limited chip clearance in the shallow grooves caused severe labyrinth seal galling and seizing when the running clearances were lost due to the accumulated structural deformations, initial misalignment, and bowing of the shaft.

Remedial Action

Replacement shafts are being fully annealed and stress relieved at $900^{\circ} \mathrm{F}$ prior to a final grinding. The gap clearance of the labyrinth seal located at the bottom of the shleld plug is being increased to 1/8". Pump M-2 will be removed for repair and modification following completion of wet criticals.

\section{HALIAM NUCLEAR FOWER FACILITY PUMPS}

\section{Pump Description}

The primary sodium pumps are vertically mounted, centrifugal, free surface type as shown in FIg. 7. The pump cases are premanently mounted in shielded cells and the pump shafts extend upward thru the shielding to the motors. The design permits the removal of internal parts, including impeller, shaft, gland and bearings through the top without disturbing the pump case or piping. The pumps are driven by an A-C induction motox through a variable speed electromagnetic coupling.

Pump Design Characteristics

$\begin{array}{ll}\text { Capacity (GPM) } & 7200 \\ \text { Total dymani.c hear (rt IIa) } & 160 \\ \text { NPSH (It Na) requijec } & 17 \\ \text { available } & 25.5 \\ \text { Case design pressure (psig) } & 100 \\ \text { Case design temperature OF } & 1000\end{array}$




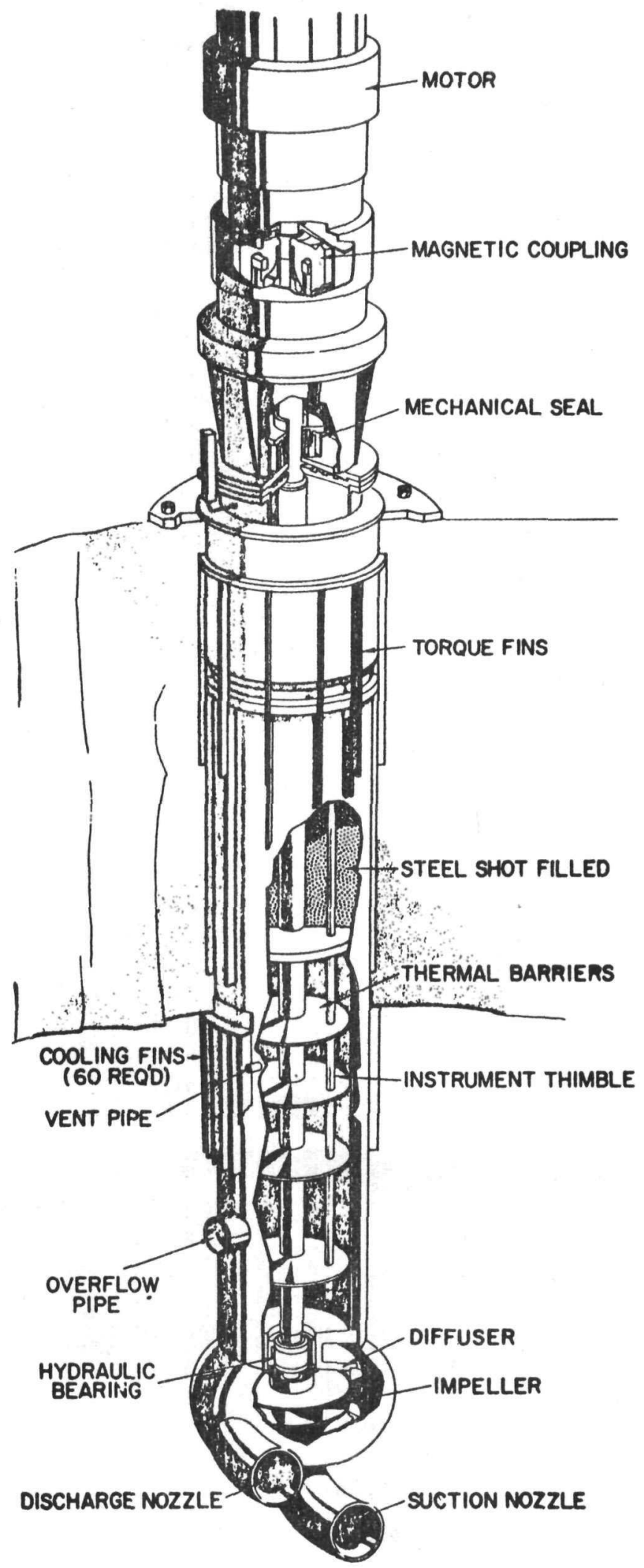

Fi.g. 7

Hallam Type Pump 
Prototypes of the Hallam pumps were tested extensively prior to specification of the pumps for the reactor. (Reference 2) All three reactor pumps were tested at rated speed and temperature at the Santa Susanna Facility before they were installed at Hallam.

\section{Operating History}

The pumps wert installed and operated for over 2000 hours without any difficulties except for an intermittent squeal. Careful examination and complete disassembly or one pump indicated no mechanical difficulties. No speciflc cause of the squeal was determined. It was believed the noise was "loop nolse" due to some resonance in the piping system.

The pumps continued to operate satisfactorily until a period shortly after a welding repair on the sodium heat exchangers. Subsequent to this repair work all three primary sodium pumps failed within a short time interval.

\section{Cause of Failure}

Disassembly of the pumps revealed that the fallure was due to forelgn material lodged in the close wear ring clearances. The source of the foreign materlal has not been definitely established but is believed to have resulted from the heat exchanger repalrs which required cutting and welding work.

During the course of examination a diagonal wear pattern was also noted on the sodium bearing. This was attributed to bearing misaligmment resulting from uneven temperature distribution around the circumference of the pump casing at the bearing elcvition. The uneven temperature distribution had been discovered during operation by uneven thermocouple temperature readings in this area. In this regard it should be noted that the pump casing was designed with extermal fins for cooling with forced convection. During construction of the plant the forced air supply was not provided and cooling in the plant installation was only by natural convection. In addition, an axial flow of sodium in the annulus between the pump shaft and the casing was provided. This axial flow path was partially blocked which resulted in the uneven circumferential temperature distribution.

Remedial Action

The pumps were cleaned up and particulate removed, score marks removed and wear ring clearances increased. The pumps are now back in operation. 


\section{REFFERENCES}

1. Seim, O. and Jaross, R. Characteristics and Performance of 5000 GPM AC Linear Induction and Mechanical Centrifugal Sodium Pumps, $\mathrm{P} / 2158$, Second International Conference on Peaceful Uses of Nuclear Energy, 1958.

2. Ats, R. W., Performance of HNPF Prototype Free Surface Sodium Pump, NAA-SR-4336, June 30, 1960. 